\title{
WestVirginiaUniversity
}

THE RESEARCH REPOSITORY @ WVU

Graduate Theses, Dissertations, and Problem Reports

2019

\section{Scheduling for Cooperative Energy Harvesting Sensor Networks}

Ahmed Ammar
aabuseta@mix.wvu.edu

Follow this and additional works at: https://researchrepository.wvu.edu/etd

Part of the Systems and Communications Commons

\section{Recommended Citation}

Ammar, Ahmed, "Scheduling for Cooperative Energy Harvesting Sensor Networks" (2019). Graduate

Theses, Dissertations, and Problem Reports. 4074.

https://researchrepository.wvu.edu/etd/4074

This Dissertation is protected by copyright and/or related rights. It has been brought to you by the The Research Repository @ WVU with permission from the rights-holder(s). You are free to use this Dissertation in any way that is permitted by the copyright and related rights legislation that applies to your use. For other uses you must obtain permission from the rights-holder(s) directly, unless additional rights are indicated by a Creative Commons license in the record and/ or on the work itself. This Dissertation has been accepted for inclusion in WVU Graduate Theses, Dissertations, and Problem Reports collection by an authorized administrator of The Research Repository @ WVU.

For more information, please contact researchrepository@mail.wvu.edu. 


\title{
Scheduling for Cooperative Energy Harvesting Sensor Networks
}

\author{
Ahmed M. Abuseta Ammar \\ Dissertation submitted to the \\ Benjamin M. Statler College of Engineering and Mineral Resources \\ at West Virginia University \\ in partial fulfillment of the requirements \\ for the degree of \\ Doctor of Philosophy \\ in \\ Electrical Engineering \\ Daryl S. Reynolds, Ph.D., Chair \\ Matthew C. Valenti, Ph.D. \\ Natalia A. Schmid, Ph.D. \\ Vinod K. Kulathumani, Ph.D. \\ Erdogan Gunel, Ph.D. \\ Lane Department of Computer Science and Electrical Engineering \\ Morgantown, West Virginia \\ 2017
}

Keywords: wireless sensor networks, energy harvesting, scheduling, throughput maximization

Copyright 2017 Ahmed M. Abuseta Ammar 


\begin{abstract}
Scheduling for Cooperative Energy Harvesting Sensor Networks
\end{abstract}

Ahmed M. Abuseta Ammar

In cooperative communication networks, the source node transmits its data to the destination either directly or cooperatively with a cooperating node. When using energy harvesting technology, where nodes collect their energy from the environment, the energy availability at the nodes becomes unpredictable due to the stochastic nature of energy harvesting processes. As a result, when the source has a transmission, it cannot immediately transmit its data cooperatively with the cooperating node. It first needs to determine whether the cooperating node has sufficient energy to forward its transmission or not. Otherwise, its transmitted data may get lost. Therefore, when using energy harvesting, the challenge is for the source to schedule its transmissions whether directly or cooperatively, such that the fraction of its events (sensed data) that are successfully reported to the destination is maximized.

Hence, in this dissertation, we address the problem of cooperating node scheduling in energy harvesting sensor networks. We consider the problem for the case of a single cooperating node and the case of multiple cooperating nodes, as well as the scenarios of one-way and two-way cooperative communications. We propose a simple scheduling scheme, called feedback scheme, which enables the source to optimally schedule its transmissions whether directly or cooperatively. We show that the feedback scheme maximizes the system performance, but does not require auxiliary parameter optimization as does the-state-of-the-art scheme, i.e., the threshold-based scheme. However, the feedback scheme has the problem of overhead caused by transmitting the energy status of the cooperating node to the source. To overcome this burden, we introduce a statistical model that enables the source to estimate the energy status of the cooperating node. Because cooperation may result in the cooperating node performing worse than the source, we address this problem through fairness in the performance between the nodes in the network. In addition, we address the problem of scheduling for throughput maximization in a wireless energy harvesting uplink. We propose centralized and distributed algorithms that find the optimal solution, and we address complexity issues. Our algorithms are shown to have a linear or quadratic complexity compared to the exponential complexity of the brute force approach. Compared with cooperative transmission, our approach maximizes the network throughput such that no node's throughput is adversely affected. 


\section{Acknowledgments}

My sincere gratitude to all those who participated and shared their ideas in this work to make it possible.

First of all, I offer my sincerest gratitude to my dissertation committee chairman and advisor, Dr. Daryl Reynolds, who gave me the opportunity to work under his supervision throughout my academic journey including my Masters and Ph.D. I would like to thank him for his patience, support, knowledge, encouragement, and effort that made this dissertation happen, and made me reach this level of my education. I would also like to thank him for all the skills that I gained and upgraded under his supervision.

I am very grateful to my committee members Dr. Matthew C. Valenti, Dr. Natalia A. Schmid, Dr. Vinod K. Kulathumani, and Dr. Erdogan Gunel. Special thanks go to Dr. Matthew C. Valenti and Dr. Natalia A. Schmid who were also members of my Masters committee, and I would like to thank them and Dr. Vinod K. Kulathumani for all the knowledge that I gained from their classes that helped me in my research. My sincere thanks to Dr. Erdogan Gunel who agreed to serve on my committee in our first meeting.

I would like to dedicate this work to my father and mother to whom I owe my success after Allah. I am thankful and grateful to them for their patience, love, compassion, and support throughout my life. 


\section{Contents}

Acknowledgments $\quad$ iii

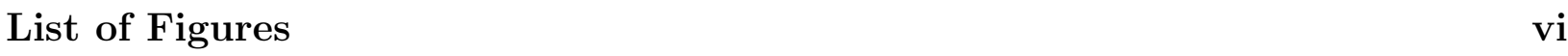

$\begin{array}{ll}\text { List of Tables } & \text { ix }\end{array}$

1 Introduction $\quad 1$

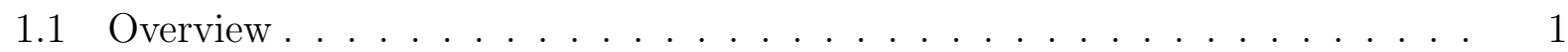

1.2 Literature Review . . . . . . . . . . . . . . . . . . . . 5

1.2.1 Energy Harvesting Sensor Networks . . . . . . . . . . . . . . . 5

1.2.2 Cooperative Energy Harvesting Sensor Networks . . . . . . . . . . . . 6

2 Single Cooperating Node Scheduling $\quad 10$

2.1 Introduction . . . . . . . . . . . . . . . . . . . . . 10

2.2 System Model . . . . . . . . . . . . . . . . . . . . . . 11

2.3 Feedback Scheduling Scheme . . . . . . . . . . . . . . . . . . . . . 14

2.4 Simulation Results . . . . . . . . . . . . . . . . . . . 16

2.4.1 Performance Evaluation . . . . . . . . . . . . . . . . . 17

2.4.2 Cooperating Node Priority Condition . . . . . . . . . . . . . . 18

2.4.3 Threshold-based Scheme Sensitivity . . . . . . . . . . . . . . . . . 19

2.5 Conclusions . . . . . . . . . . . . . . . . . . . . . . . 20

3 Multiple Cooperating Nodes Scheduling $\quad 22$

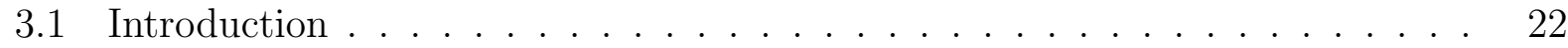

3.2 System Model . . . . . . . . . . . . . . . . . . . . . . 23

3.3 Scheduling Algorithm . . . . . . . . . . . . . . . . . . . . . . . . . . . . . . . . . . . . . . 25

3.4 Adaptive Scheme for Absolute Fairness . . . . . . . . . . . . . . . . . . . . . . . . . . . . . . . 38

3.5 Simulation results . . . . . . . . . . . . . . . . . . . . . . . . . . . . . . . . . . . . 30

3.5.1 One-Way Cooperative Scenario . . . . . . . . . . . . . . . 30

3.5.2 Two-Way Cooperative Scenario . . . . . . . . . . . . . . . . . 31

3.5.3 Overhead Reduction . . . . . . . . . . . . . . . . . . 33

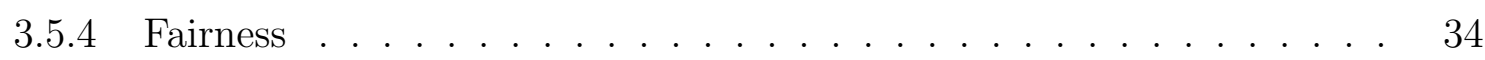

3.6 Conclusions . . . . . . . . . . . . . . . . . . . . . 35 
4 Generalized Fairness and Optimal Scheduling 38

4.1 Introduction . . . . . . . . . . . . . . . . . . . . . . 38

4.2 System Model . . . . . . . . . . . . . . . . . . . . . . . . . . . . . . . . . . . . . . . . . . . . .

4.3 Operating Modes . . . . . . . . . . . . . . . . . . . 42

4.3.1 Noncooperation Mode . . . . . . . . . . . . . . . . . 42

4.3 .2 Cooperation Mode . . . . . . . . . . . . . . . . . 43

4.3 .3 Relay mode . . . . . . . . . . . . . . . . . . . 47

4.3 .4 Mixed mode . . . . . . . . . . . . . . . . . 49

4.4 Scheduling Schemes . . . . . . . . . . . . . . . . . . . . 50

4.4.1 Fair Scheduling (FS) Scheme . . . . . . . . . . . . . . . . 50

4.4.2 Constrained Scheduling (CS) Scheme . . . . . . . . . . . . . . 55

4.5 Simulation Results . . . . . . . . . . . . . . . . . . . . . . . . . . . . . . . . . . . 56

4.5.1 Fairness . . . . . . . . . . . . . . . . . 57

4.5.2 Constrained Scheduling . . . . . . . . . . . . . . . . 59

4.6 Conclusions . . . . . . . . . . . . . . . . . . . . . . . 59

5 Scheduling with Energy Status Estimation $\quad 61$

5.1 Introduction . . . . . . . . . . . . . . . . . . . . 61

5.2 Cooperation Mode Statistical Model . . . . . . . . . . . . . . . . . 62

5.3 Relay Mode Statistical Model . . . . . . . . . . . . . . . . . . . 66

5.4 Simulation Results . . . . . . . . . . . . . . . . . . 66

5.4.1 Cooperation and relay modes (independent case) . . . . . . . . . 67

5.4.2 Cooperation and relay modes (correlated case) . . . . . . . . . 68

5.4 .3 FS and CS schemes . . . . . . . . . . . . . . . 69

5.5 Conclusions . . . . . . . . . . . . . . . . . . . . 71

6 Scheduling for Throughput Maximization $\quad 72$

6.1 Introduction . . . . . . . . . . . . . . . . . . . . . 72

6.2 System Model . . . . . . . . . . . . . . . . . . . . . . . . . . . . . . . . . . 74

6.3 Successful transmission probability Characterization . . . . . . . . . . . 76

6.4 Problem Formulation . . . . . . . . . . . . . . . . . . . . . . . 77

6.5 A brute force approach . . . . . . . . . . . . . . . . . . . . . . 79

6.6 Centralized Algorithm . . . . . . . . . . . . . . . . . . . . . 80

6.7 Distributed Algorithm . . . . . . . . . . . . . . . . . . . . . . . . . . . . . . . . . . . . . . . . . . . . 84

6.8 Variable Transmit Power . . . . . . . . . . . . . . . . . . . . . . . . . . . . . . . . . 88

6.9 Simulation Results . . . . . . . . . . . . . . . . . . . . . . . . 89

6.9.1 Fixed Transmit Power . . . . . . . . . . . . . . . . . . . . . 92

6.9.2 Variable Transmit Power . . . . . . . . . . . . . . . . . . . . . 92

6.9.3 Comparison with Cooperative Transmission . . . . . . . . . . . . . 94

6.10 Conclusions . . . . . . . . . . . . . . . . . . . . . 95

7 Summary and Future Work 96

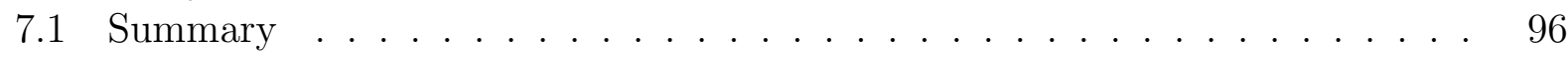

7.2 Future Work . . . . . . . . . . . . . . . . . . . 98

$\begin{array}{ll}\text { References } & 101\end{array}$ 


\section{List of Figures}

2.1 A three-node network with an energy harvesting source $N_{1}$, an energy harvesting cooperating node $N_{2}$, and a destination. $E_{1}$ and $E_{2}$ are queues representing the energy harvested by $N_{1}$ and $N_{2}$, respectively, in the $n$-th time block, $n \in\{1,2, \ldots, N\}$. $A$ is the latest energy status of the cooperating node

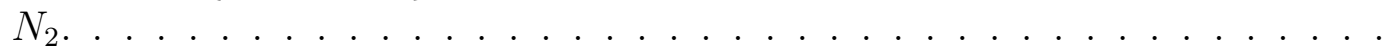

2.2 A state diagram of the two-state Markov process that models the event generation process of node $N_{i}, i \in\{1,2\}$ with transition probabilities $q_{i}^{\text {on }}$ and $q_{i}^{\text {off }}$. The energy generation process is modeled the same but with transition probabilities $p_{i}^{\text {on }}$ and $p_{i}^{\text {off }}$.

2.3 Two flowcharts illustrate the feedback and the threshold-based schemes to easily notice the difference between them. . . . . . . . . . . . . . . .

2.4 The overall, the cooperating node, and the source packet delivery ratios of the feedback and the threshold-based schemes. Parameters used: $q_{1}^{\text {on }}=0.9$, $q_{1}^{\text {off }}=0.7, p_{1}^{\text {on }}=q_{2}^{\text {on }}=0.85, p_{1}^{\text {off }}=q_{2}^{\text {off }}=0.7, E_{1}=E_{2}=1, \delta_{1}=\delta_{2}=3$, $\tilde{\delta}_{1}=\tilde{\delta}_{2}=1, K=20, T_{H}=10 \ldots \ldots \ldots \ldots \ldots \ldots \ldots \ldots \ldots$

2.5 The overall, the cooperating node, and the source packet delivery ratios of the feedback scheme with both the source and the cooperating node priority conditions. Parameters used: $q_{1}^{\text {on }}=0.9, q_{1}^{\text {off }}=0.7, p_{1}^{\text {on }}=q_{2}^{\text {on }}=0.85, p_{1}^{\text {off }}=$ $q_{2}^{\text {off }}=0.7, E_{1}=E_{2}=1, \delta_{1}=\delta_{2}=3, \tilde{\delta}_{1}=\tilde{\delta}_{2}=1, K=20, T_{H}=10 . \ldots$.

2.6 The overall, the cooperating node, and the source packet delivery ratios of the feedback and the threshold-based schemes when changing one of the parameters. Parameters used: $q_{1}^{\text {on }}=0.9, q_{1}^{\text {off }}=0.7, p_{1}^{\text {on }}=q_{2}^{\text {on }}=0.85, p_{1}^{\text {off }}=q_{2}^{\text {off }}=0.7$, $E_{1}=E_{2}=1, \delta_{1}=3, \delta_{2}=\tilde{\delta}_{1}=\tilde{\delta}_{2}=1, K=20, T_{H}=10 \ldots \ldots \ldots$

3.1 Energy harvesting sensor network of $M-2$ cooperating nodes that assist cooperative communication between two sensors $\left(N_{1}, N_{2}\right)$. Each node $N_{m}, m \in$ $\{1,2, \ldots, M\}$ harvests $E_{m}$ amount of energy if its energy generation process is "on". Each node has a memory of $M-2$ slots to save the latest status, $A_{m}, m \in\{3,4, \ldots, M\}$, of the cooperating node $N_{m}$ as either 1 or 0 in the corresponding $m$-th slot. . . . . . . . . . . . . . .

3.2 A flowchart illustrates the feedback scheme for the case of multiple cooperating nodes when the cooperating node of the highest index is selected for relay transmission. 
3.3 The packet delivery ratios of the one-way scenario when $M=3$ and $M=4$. Parameters used: $q_{1}^{\text {on }}=0.9, q_{1}^{\text {off }}=0.7, p_{1}^{\text {on }}=q_{3}^{\text {on }}=0.85, p_{1}^{\text {off }}=q_{3}^{\text {off }}=0.7$, $E_{1}=E_{3}=1, \delta_{1}=\delta_{3}=3, \tilde{\delta}_{1}=\tilde{\delta}_{3}=1, K=20 \ldots \ldots \ldots \ldots$

3.4 The packet delivery ratios of the one-way and two-way scenarios when $M=3$. Parameters used: $q_{1}^{\text {on }}=0.9, q_{1}^{\text {off }}=0.7, p_{1}^{\text {on }}=q_{3}^{\text {on }}=0.85, p_{1}^{\text {off }}=q_{3}^{\text {off }}=0.7$, $E_{1}=E_{3}=1, \delta_{1}=\delta_{3}=3, \tilde{\delta}_{1}=\tilde{\delta}_{3}=1, K=20 \ldots \ldots \ldots \ldots \ldots$

3.5 The packet delivery ratios of the one-way and two-way scenarios when $M=4$. Parameters used: $q_{1}^{\text {on }}=0.9, q_{1}^{\text {off }}=0.7, p_{1}^{\text {on }}=q_{3}^{\text {on }}=0.85, p_{1}^{\text {off }}=q_{3}^{\text {off }}=0.7$, $E_{1}=E_{3}=1, \delta_{1}=\delta_{3}=3, \tilde{\delta}_{1}=\tilde{\delta}_{3}=1, K=20 \ldots \ldots \ldots \ldots$

3.6 The number of status transmissions of the standard and adaptive status broadcastings for the one-way scenario when $M=3$. Parameters used: $q_{1}^{\text {on }}=0.9$, $q_{1}^{\text {off }}=0.7, p_{1}^{\text {on }}=q_{3}^{\text {on }}=0.85, p_{1}^{\text {off }}=q_{3}^{\text {off }}=0.7, E_{1}=E_{3}=1, \delta_{1}=\delta_{3}=3$, $\tilde{\delta}_{1}=\tilde{\delta}_{3}=1, K=20$.

3.7 The packet delivery ratios of the two ways of cooperating nodes selection (selecting the node of the highest index and selecting the nodes sequentially) for the one-way scenario when $M=4$. Parameters used: $q_{1}^{\text {on }}=0.9, q_{1}^{\text {off }}=0.7$, $p_{1}^{\text {on }}=q_{3}^{\text {on }}=0.85, p_{1}^{\text {off }}=q_{3}^{\text {off }}=0.7, E_{1}=E_{3}=1, \delta_{1}=\delta_{3}=3, \tilde{\delta}_{1}=\tilde{\delta}_{3}=1$, $K=20$.

3.8 The convergence of the packet delivery ratios for the one-way and two-way scenarios when $M=3$. Parameters used: $q_{1}^{\text {on }}=q_{3}^{\text {on }}=1, q_{1}^{\text {off }}=q_{3}^{\text {off }}=0$, $p_{1}^{\text {on }}=0.85, p_{3}^{\text {on }}=0.95, p_{3}^{\text {off }}=0.55, p_{1}^{\text {off }}=0.7, E_{1}=E_{3}=1, \delta_{1}=3$, $\tilde{\delta}_{1}=\tilde{\delta}_{3}=\delta_{3}=1, K=20$.

4.1 A cooperative energy harvesting sensor network of two transmitting nodes $\left(N_{1}\right.$ and $\left.N_{2}\right)$ and a destination. $E_{1}$ and $E_{2}$ are queues representing the energy harvested by $N_{1}$ and $N_{2}$, respectively, in the $n$-th time block, $n \in\{1,2, \ldots, N\}$.

4.2 The figure illustrates the noncooperation, cooperation, and relay modes, which are used in appropriate combinations to build the FS and CS scheduling schemes. The solid line between $S_{1}, S_{2}$, or cooperating node and destination represents the direct transmission of the event of that node to the destination. The dashed path in the cooperation and relay modes represents the relay transmission of $S_{2}$ to the destination using cooperating and relay nodes, respectively. In the relay mode, there is no solid line between the relay node and the destination because the relay node has no own transmissions where it drops all its transmissions to help with $S_{2}$ transmissions. . . . . . . . . .

4.3 The packet delivery ratios of $N_{1}$ and $N_{2}$ in noncooperation mode. Parameters used: $q_{1}=0.5, q_{2}=0.4, \tilde{\delta}=1, \delta_{1}=\delta_{2}=2, \tilde{K}=1, K=100, N=5 \times 10^{5}$.

4.4 Figures (a) and (b) illustrate the optimal $x$ and $n$ provided by the FS scheme when $\alpha=1$. Figure (c) shows the optimal performance of $N_{1}$ and $N_{2}$ by which fairness is considered achieved for the given penalty function. . . . . .

4.5 Figures (a) and (b) illustrate the optimal $x$ and $n$ provided by the FS scheme when $\alpha=3$. Figure (c) shows the optimal performance of $N_{1}$ and $N_{2}$ by which fairness is considered achieved for the given penalty function. . . . . . 
4.6 Figures (a) and (b) illustrate the optimal $x$ and $n$ provided by the FS scheme when $\alpha=5$. Figure (c) shows the optimal performance of $N_{1}$ and $N_{2}$ by which fairness is considered achieved for the given penalty function. . . . . .

4.7 Figures (a) and (b) illustrate the optimal $x$ and $n$ provided by the CS scheme in order for node $N_{1}$ to achieve the performance $\gamma=0.7$. Figure (c) shows that node $N_{1}$ achieves the performance $\gamma$ over the values $\overline{E_{1}}$ and $\overline{E_{2}}$ where the system is feasible. . . . . . . . . . . . . . . . . .

5.1 Figures (a) and (b) show the performance of $N_{1}$ in the cooperation and relay modes using the statistical models compared with the feedback scheme. Figure (c) shows the number of energy status transmissions of $N_{2}$ over the $N$ time blocks.

Figures (a) and (b) show the packet delivery ratio of $N_{1}$ versus correlation coefficient $\rho$ for the cooperation and relay modes, respectively, using the statistical models compared with the feedback scheme. . . . . . . . . . . . .

5.3 Figures (a) and (b) show the packet delivery ratios of $N_{1}$ and $N_{2}$, respectively, for the FS scheme when using the statistical models compared with the feedback scheme. We used the same penalty function in Section VI (A) with

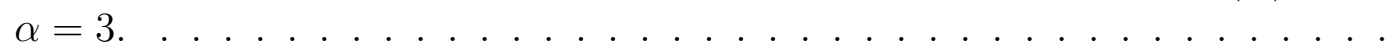

5.4 Figures (a) and (b) illustrate the optimal $x$ and $n$ provided by the CS scheme using the statistical models in order for node $N_{1}$ to achieve the performance $\gamma=0.7$. Figure (c) shows that node $N_{1}$ achieves the performance $\gamma$ over the values $\overline{E_{1}}$ and $\overline{E_{2}}$ where the system is feasible. . . . . . . . . . . . . .

6.1 A network consists three categories of sensor nodes; type $S_{1}$ node which transmits directly to the base station, type $S_{2}$ node which transmits to the base station through another node called cooperating node, and cooperating node which transmits its own events as well as it forwards some type $S_{2}$ nodes' events to the base station. . . . . . . . . . . . . . . . .

6.2 Successful transmission probability $\tau_{i}\left(q_{i}, \delta_{i}, \boldsymbol{p}_{i}\right)$ of node $i \in \mathcal{N}$ versus $q_{i}$ for different values of $\delta_{i}$ and $K$, and for $\boldsymbol{p}_{i}=\left[\begin{array}{ll}0.3 & 0.7\end{array}\right] \ldots \ldots \ldots$.

6.3 The number of all possible combinations increases exponentially with $M$. . .

6.4 Figure (a) shows the network throughput for all the scenarios shown in Table 6.1. Figure (b) shows the successful transmission probability of $N_{1}, N_{2}$, and $N_{3}$. Parameters used: $q_{1}=q_{2}=q_{3}=0.2, \boldsymbol{p}_{1_{\tilde{\delta}}}=\boldsymbol{p}_{2}=\left[\begin{array}{ll}0.2 & 0.8\end{array}\right], \boldsymbol{p}_{3}=\left[\begin{array}{ll}0.8 & 0.2\end{array}\right]$, $\tilde{K}=1, K=100, N=10^{5}, \delta_{1}=\delta_{2}=\delta_{3}=2, \tilde{\delta}=1 \ldots \ldots \ldots \ldots$

6.5 Figure (a) shows the network throughput for all the scenarios shown in Table 6.1. Figure (b) shows the successful transmission probability of $N_{1}, N_{2}$, and $N_{3}$. Parameters used: $q_{1}=q_{2}=q_{3}=0.2, \boldsymbol{p}_{1}=\boldsymbol{p}_{2}=\boldsymbol{p}_{3}=\left[\begin{array}{ll}0.2 & 0.8\end{array}\right]$, $\tilde{K}=1, K=100, N=10^{5}, \delta_{1}=\delta_{2}=\delta_{3}=2, \tilde{\delta}=1 \ldots \ldots \ldots \ldots$

6.6 The network throughput for each scenario shown in Table 6.1 when the transmit power is variable and fixed. Parameters used: $q_{1}=0.2, q_{2}=0.8, q_{3}=0.2$, $\boldsymbol{p}_{1}=\left[\begin{array}{llllll}0.025 & 0.025 & 0.025 & 0.025 & 0.1 & 0.8\end{array}\right], \boldsymbol{p}_{2}=\left[\begin{array}{llllll}0.2 & 0.3 & 0.3 & 0.2 & 0 & 0\end{array}\right], \boldsymbol{p}_{3}=\left[\begin{array}{lll}0.1 & 0.1\end{array}\right.$ $0.20 .30 .30], \tilde{K}=5, K=100, N=10^{5}, \delta_{1}=\delta_{2}=\delta_{3}=6, \delta_{1,2}=2, \delta_{1,3}=4$, $\delta_{2,3}=1$. 


\section{List of Tables}

6.1 All Possible Scenarios of the Network . . . . . . . . . . . . . . . . . . 90 


\section{Chapter 1}

\section{Introduction}

\subsection{Overview}

Wireless sensor networks have been widely used for different applications including surveillance and monitoring, medical, and emergency applications [1]. Commonly, these applications use sensors that are powered by small size batteries so that they become energy-limited. One general approach to improving energy efficiency in sensor network applications is cooperative communications, in which source node's packets are sent to the destination through direct paths and through relays/cooperating nodes [2]-[6]. Existing research has shown that cooperative communications can achieve the same bit error rate with a lower transmission energy as compared to direct transmissions [7].

Importantly, the limited size of the batteries causes the demand of periodic batteries replacement or recharging, which is costly, inconvenient, or sometimes impossible, especially in networks of a high number of sensors that are deployed in a hostile environment. One solution to this problem, energy harvesting, has recently generated a great deal of research interest for wireless sensor networks $[8,9]$. Energy harvesting can provide essentially limitless energy without the hassle of manual recharging, battery replacement, and the dangers associated with battery leakage (environmentally-friendly). Moreover, energy harvesting can be used in emergencies when a conventional power supply is unavailable or human intervention is impractical.

These advantages of energy harvesting and the low power transmission property of co- 
operative communications have motivated researchers to combine these two technologies in wireless sensor networks. Thus, their energy efficiency increases, and they become well suited in some applications where human intervention or battery replacement/recharging is impractical. However, the stochastic nature of the energy harvesting processes imposes more challenges on the system design [10]. One of the challenges is cooperating node usage scheduling for cooperative communication. Due to the stochastic energy availability at the nodes, the source cannot immediately transmit cooperatively with the cooperating node. It has first to determine whether the cooperating node has sufficient energy to forward its transmission or not. Otherwise, its transmission may get lost. Under this energy constraint, the challenge here is how to design an efficient scheduling algorithm that enables the source to optimally schedule its transmission whether on its own or by using the cooperating node, such that the packet delivery ratio of the system is maximized. In this dissertation, we focus on this problem.

First, in Chapter 2, we address the problem of single cooperating node scheduling. We consider a network of three nodes; a source, a cooperating node, and a destination, where the source transmits its events either directly or cooperatively using the cooperating node to the destination, and the cooperating node helps with the source's transmissions as well as it transmits its own events to the destination. For such a network model, we propose a scheduling algorithm, called feedback algorithm, that enables the source to optimally decide whether to transmit directly or cooperatively such that the packet delivery ratio of the network is maximized, which is the ratio of the total events that are successfully delivered, to the total events that are generated. In the proposed algorithm, the cooperating node usage is scheduled based on a feedback that is frequently sent to the source carrying the energy status of the cooperating node as either 1 or 0, i.e., energized or unenergized, where energized means that the cooperating node has sufficient energy to forward the source's transmission to the destination. When the source has a transmission and the cooperating node status is energized, cooperating node usage is scheduled; otherwise the source is scheduled to transmit on its own if it has sufficient energy for direct transmission. The results show that our algorithm maximizes the system performance, but does not require auxiliary parameter optimization as does the-state-of-the-art scheme, which is the threshold-based scheme [53]. 
In the threshold-based scheme, the decision on the cooperating node usage is made based on a threshold level. When the source has a transmission and its energy battery level is less than that threshold level, the cooperating node is scheduled to assist with the source's transmission. Otherwise, the source is scheduled to transmit on its own. To maximize the system performance, the threshold level must be tuned for any given set of system parameters or for any change in the system parameters. Tuning the threshold level requires simulating the system at different energy levels of the source's battery. This might not be easy or even possible to implement especially in networks of unstable parameters or in networks with sensors of a huge battery capacity.

Next, in Chapter 3 we upgrade the feedback algorithm for the scenario of multiple cooperating nodes and two-way cooperative communications. In this scenario, we assume that the system has multiple cooperating nodes between two transmitting nodes, which communicate with each other either directly or cooperatively using one of the cooperating nodes. In the algorithm, the cooperating nodes are indexed increasingly, and each node is assumed to broadcast its energy status to the network as either energized or unenergized whenever it changes. Hence, when any of the transmitting nodes has a transmission and there is at least one energized cooperating node, the transmitting node transmits cooperatively. Otherwise, it transmits directly if it has sufficient energy for direct transmission. If there is more than one energized cooperating node, the transmitting node uses the cooperating node of the highest index, or in another scenario, it uses the energized cooperating nodes sequentially. Because of the overhead problem caused by broadcasting the energy status of each cooperating node to the network, we propose an adaptive method to decrease this burden. That is, when the energy harvesting rate of a cooperating node is low, the other nodes in the network will set the energy status of that node to unenergized after each time it transmits as it will be more likely to be so. If the node is still energized after transmission, the node will broadcast 1 to the network so that the other nodes will set its status back to energized. Thus, the cooperating node does not need to always broadcast its energy status to the network, which will reduce the overhead when the energy harvesting rate is low. In addition, we propose an adaptive scheduling algorithm that incorporates fairness constraints to ensure the same performance between the nodes. The algorithm optimally 
switches between two conditions, where one prioritizes the source's transmission while the other prioritizes the cooperating node's transmission, such that the two nodes achieve the same performance. However, in the adaptive scheme, the penalty on the cooperating node performance sometimes has to be high in order to achieve the same performance of the source node. In addition, because in the system model each node is fixed and categorized a priori as either a source or a cooperating node, in the adaptive scheme fairness cannot be achieved if the source has higher performance than the cooperating node. Therefore, in Chapter 4, we first generalize the system model of the three nodes network, where each node can be either a source, a cooperating, or a relay node depending on the system parameters. Then, We propose a fairness scheduling (FS) scheme that achieves fairness based on a given penalty function, which fairly determines how much a node should cooperate with the other. We also propose a constrained scheduling (CS) scheme that constrains one of the nodes to achieve a certain performance.

Although the adaptive method proposed in Chapter 3 decreases the burden of overhead, it is still relatively high and could consume a significant amount of energy, especially when using protocols of fixed packet/frame format, as in IEEE 802.11. Thus, to solve the problem of overhead, we introduce in Chapter 5 a statistical model that estimates the cooperating node's energy status at the source. The simulation results show that over some values of the energy harvesting rates of the transmitting nodes, it is more energy efficient to use the statistical model than the feedback scheme with the adaptive method. Lastly, we address in Chapter 6 the problem of scheduling for throughput maximization in a wireless energy harvesting uplink. In most of the existing works, throughput is maximized at the expense of some nodes that are either close to the sink or that discard their own transmissions in favor of relaying. For fairness, we maximize the network throughput such that each node's throughput cannot drop below what it achieves by direct transmission to the base station. The throughput is maximized using data relaying, and by optimally assigning a role to each node, i.e., cooperating node, source (direct transmission), or user (of a cooperating node). Both centralized and decentralized algorithms that find the optimal assignment of each node are proposed and studied. We consider both fixed and variable transmit power scenarios, and address complexity issues. Our algorithms are shown to have a linear or 
quadratic complexity compared to the exponential complexity of the brute force approach. Compared with cooperative transmission, our approach maximizes the overall throughput of the network such that no node's throughput is adversely affected.

Throughout this dissertation, we assume a real-time monitoring scenario, where no packets/events are buffered for later transmission. In other words, we assume that there is no buffer for storing sensed data as in [71]. We also assume that the transmission energy required for reliably transmitting an event from a sensor node to another or from a sensor node to a destination is constant, i.e., the transmission rate is constant. Moreover, unlike [63], we assume that if a node has an event to transmit to the destination, the node transmits only if its current residual energy is sufficient for reliable transmission. Thus, if the transmission occurs, the transmitted event is considered successfully delivered to the destination, with no retransmissions required. We do not consider the energy consumption of signal processing including receive signal processing. However, it can easily be subsumed under the transmit energy.

\subsection{Literature Review}

In this section, we present literature related to the problems addressed in this dissertation. We first start with the research work related to the area of energy harvesting sensor networks, where we briefly discuss the technology of energy harvesting and mention some research work that consider this technology in wireless sensor networks. Then, we present the research work in the area of using cooperative communication in energy harvesting sensor networks. In this area, we first talk about cooperative communication technology, and then we present some research work that combine this technology with energy harvesting in wireless sensor networks.

\subsubsection{Energy Harvesting Sensor Networks}

Energy harvesting technology has been widely addressed in the literature. Some of its sources have been studied and investigated including but not limited to wind, solar, thermal, and mechanical vibration [11]. Depending on the energy source, it has been shown that the 
power that can be harvested is in the range between several $\mu W$ to several hundred $m W$ $[12,13,14]$. Because the power consumption of a sensor node is usually in the range between 1 to $100 \mu W[15,16,17]$, in the past few years some sensor nodes that can be powered by energy harvesting sources have been introduced to the research communities. Some of the existing energy harvesting sensor nodes are listed in [18]. Such sensor nodes can provide many unique features that cannot be provided by conventional sensors, including, 1) no need for battery replacement/recharging when it runs out, which reduces labor and new batteries' costs, 2) easy and fast deployment in hostile or inaccessible environments, and 3) reduction of carbon effects associated with batteries leakage. These attributes have motivated researchers to study and consider the idea of using energy harvesting technology in wireless sensor networks. Due to the new imposed energy constraints, some fundamental issues and standard designs of wireless sensor networks have been revisited and reanalyzed in the literature. In [19] and [20], the authors analyzed the performance of various MAC schemes for a multi-sink and a single-sink networks. In [21], the authors analyzed the power consumption of a ZigBeeBased energy harvesting sensor network. The authors in [22] investigated the feasibility of using IEEE 802.11 in energy harvesting sensor networks of low-power sensing applications. In [23], the authors characterized the transmission capacity of an ad hoc wireless network with ALOHA and CSMA protocols. Some sleep and wakeup strategies have been proposed for solar-powered wireless sensor networks in [24]. Finally, the authors in [25]-[30] have revisited the issues of power transmission and allocation as well as the issue of throughput maximization for different models of energy harvesting sensor networks. More research work and challenges can be found in [10].

\subsubsection{Cooperative Energy Harvesting Sensor Networks}

The idea of cooperative communication was first introduced by van der Meulen in the 1970s, where he introduced the three-terminal relay channel and derived the inner bounds for capacity using a timesharing approach [5]. The results in this paper showed that cooperative communication can be a promising solution for communication systems. Therefore, after that, cooperative communication has been addressed extensively in the literature. Different 
issues were investigated and analyzed including diversity gain, outage probability, capacity, energy and bandwidth utilization. In [6], the authors analyzed the capacity for the threeterminal relay channel assuming that all the nodes operate in the same band. In [31] and [32], the authors explored the idea of some general cooperative communication scenarios including decode-and-forward, amplify-and-forward, and selection relaying. The idea of cooperative communication was extended further to a more generic sense of cognitive radio in [33]. In [34]-[36], the authors focused on achieving diversity gains in coded cooperation systems. Cooperative communication using collaborative MIMO has also been investigated in the literature. Some recent developments and challenges about using cooperative MIMO in sensor networks can be found in [37].

Cooperative communication has been used in wireless sensor networks for more energy efficiency and longer lifetime. However, some fundamental issues have been revisited and considered. In [38]-[41], some MAC layer issues have been considered and cooperative MAC protocols have been proposed under different assumptions. A cross-layer strategy and a cooperation algorithm were proposed in [42] and [43], respectively. Cooperative communication has also been used in wireless sensor networks with energy harvesting to maximize the data collected from their sensor nodes. Data collection can also be maximized using a mobile sink [44]-[46] or multihop transmission [47]-[52]. However, using a mobile sink may not be suitable for small networks or for hostile environments, where optimizing the trajectory may be impossible. On the other hand, using mutlihop transmission results in data delivery latency that may be untolerated in some applications. These two problems are avoided when using cooperative communication.

In the literature, there has been a substantial research effort on using cooperative communication to maximize data collection in energy harvesting sensor networks. In [53], the authors considered a three-node relay network, and developed a scheduling scheme to maximize the data collected from the source node. In [54]-[58], the problem was considered for the scenario when the network has multiple relays, and relay selection protocols were proposed. The authors in [59] and [60] considered the problem for a three-node network of an energy harvesting relay node that harvests its energy from the RF radiation of the source. The authors proposed relaying protocols that enable the relay to switch between 
energy harvesting and data processing. A relaying protocol was also proposed in [61] for a different setup of three-node relay network. In [62], the problem was considered for a Gaussian three-node relay network. The authors in [63] improved data throughput for a network of a set of nodes over low energy harvesting rates. The network throughput is improved by assigning a cooperative repeater role to some nodes, which drop all their transmissions over a period of time and retransmit the sources' transmissions only if they are not successfully received at the base station. Lastly, in order to maximize the data collected from the source at the destination, the authors in [64] proposed schemes that enable the source to optimally schedule the relays' energy harvesting and data transmission for a multiple-relay network.

As in [53], in this dissertation, we also consider the problem of relay/cooperating node usage scheduling for cooperative communication at source. Using partially observable Markov decision process, the authors in [53] derived an optimal policy which maximizes the system performance in terms of the packet delivery ratio, which is the ratio of the data that is successfully delivered, to the total data that is generated. This optimal policy is quite sensitive to system parameters, which makes it impractical. Therefore, the authors designed a simple and practical threshold-based relay scheduling scheme that is sensitive only to one system parameter. The scheme achieves performance close to the optimal policy, and thus we consider it as the state-of-the-art scheme to evaluate our proposed schemes. Because cooperative communication can maximize data collection at the expense of relay or cooperating nodes, we also use the approach of data relaying in the last chapter to maximize the network throughput such that no node's throughput is adversely affected. In this approach, each node is assumed to transmit to the base station either direct or in two hops.

The rest of the dissertation is organized as follows. Chapter 2 addresses the problem of single cooperating node scheduling, and proposes a practical relay scheduling scheme to maximize the system performance. In Chapter 3, the proposed scheme in Chapter 2 is upgraded to include the scenarios of multiple cooperating nodes and sources, and to accommodate fairness constraints. Chapter 4 generalizes fairness and provides analyticalbased approach fair and constraint scheduling schemes for optimal scheduling. In Chapter 5, a statistical model is proposed to estimate the energy status of the relay or the cooperating node, considering the same system model in Chapter 4. Chapter 6 considers the problem of 
data collection throughput maximization for an uplink wireless network. Chapter 7 concludes the dissertation and presents some directions for future work. 


\section{Chapter 2}

\section{Single Cooperating Node Scheduling}

\section{$2.1 \quad$ Introduction}

In this chapter, we consider a network of a single cooperating node that assists cooperative communication between a source and a destination. We propose a simple practical scheduling scheme, called feedback scheme, that enables the source to optimally decide whether to transmit on its own or by using the cooperating node in order to maximize the packet delivery ratio of the network, which is the ratio of the data that is successfully delivered, to the total data that is generated. In the feedback scheme, the decision on the cooperating node usage is made based on its actual energy status that is sent to the source in a feedback message as either 1 or 0, i.e., energized or unenergized, where energized means that the cooperating node has sufficient energy to forward the source's transmission. The results show that the feedback scheme provides almost the same performance of the threshold-based scheme, which performs close to the optimal policy derived in [53]. The threshold-based scheme is sensitive to one of the system parameters, threshold level. In order to maximize the system performance, the threshold level has to be tuned for each given set of parameters or for any change in the parameters, and tuning the threshold level requires simulating the system at different levels of the source battery. Therefore, as the feedback scheme does not require auxiliary parameter optimization to maximize the system performance, it becomes more practical especially in networks with unstable parameters or with sensors of large battery capacity.

Portion of this chapter appears in: A. Ammar and D. Reynolds, "A practical relay scheduling scheme for wireless sensor networks with energy harvesting," 47th Annual Conference on Information Sciences and Systems (CISS), vol. 1, no. 6, pp. 20-22, March 2013. 


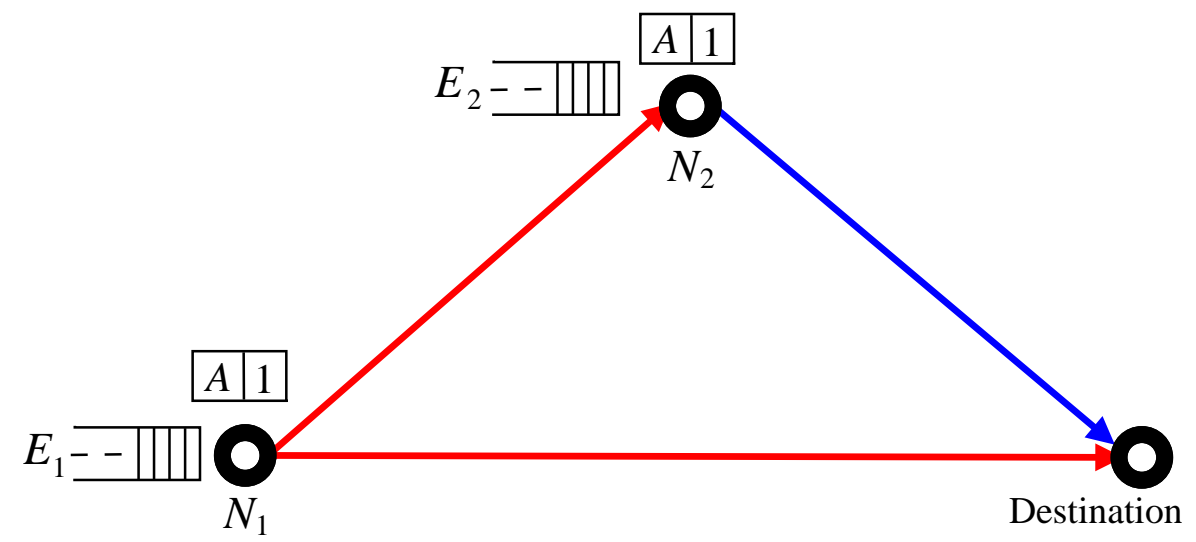

Figure 2.1: A three-node network with an energy harvesting source $N_{1}$, an energy harvesting cooperating node $N_{2}$, and a destination. $E_{1}$ and $E_{2}$ are queues representing the energy harvested by $N_{1}$ and $N_{2}$, respectively, in the $n$-th time block, $n \in\{1,2, \ldots, N\}$. $A$ is the latest energy status of the cooperating node $N_{2}$.

\subsection{System Model}

We consider a three-node network with a source node $N_{1}$, a cooperating node $N_{2}$, and a destination, as depicted in Figure 2.1, where each node is equipped with energy harvesting devices and has a rechargeable battery with capacity $K$. We assume a discrete-time model, where time is divided into $N$ time blocks. To avoid collision, we use a TDMA protocol where each block is divided into three time slots. The first time slot is for the source's transmission, the second is for the cooperating node to forward the source's transmission, and the third is for the cooperating node's transmission. The energy available at node $N_{i}, i \in\{1,2\}$ in the time block $n, n \in\{1,2, \ldots, N\}$ is denoted by $L_{i}[n] \in\{0,1,2, \ldots, K\}$.

We assume that there is at most one event generated per each transmitting node $N_{1}$ and $N_{2}$ per time block. As in [53] and [65], the event generation process at node $N_{i}, i \in\{1,2\}$ is modeled by a temporally correlated, two-state Markov process ("on", "off") with transition probabilities $q_{i}^{\text {on }}$ and $q_{i}^{\text {off }}$, as illustrated in Figure 2.2. In the time block $n, n \in\{1,2, \ldots, N\}$, the "on" state means that there is an event generated at that block, i.e., $\mathcal{E}_{i}[n]=1$, while the "off" state means that there is no event generated, i.e., $\mathcal{E}_{i}[n]=0$. The energy generation process of $N_{i}, i \in\{1,2\}$ is assumed to be modeled by the same process, but with transition probabilities $p_{i}^{\text {on }}$ and $p_{i}^{\text {off }}$. The "on" state at the $n$-th time block means that the sensor 


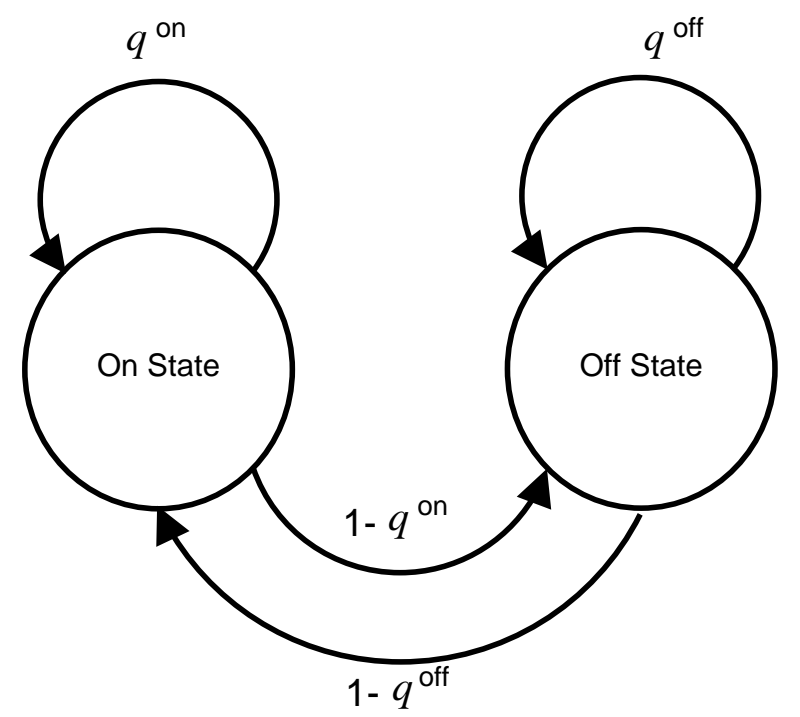

Figure 2.2: A state diagram of the two-state Markov process that models the event generation process of node $N_{i}, i \in\{1,2\}$ with transition probabilities $q_{i}^{\text {on }}$ and $q_{i}^{\text {off }}$. The energy generation process is modeled the same but with transition probabilities $p_{i}^{\text {on }}$ and $p_{i}^{\text {off }}$.

harvests $E_{i}$ amount of energy at that block while the "off" state means that there is no energy harvested.

During each time block in which there is an event to report, the source node $N_{1}$ operates in one of two transmission modes; direct transmission or relay transmission. In direct transmission, the source transmits its event directly to the destination using $\delta_{1}$ amount of energy. In relay transmission, the source transmits its event to the destination using $\tilde{\delta}_{1}$ amount of energy, and it also makes use of the cooperating node $N_{2}$ to forward its transmission to the destination in the next time slot. The cooperating node $N_{2}$ also operates in one of two transmission modes; own-traffic transmission or relay transmission. In the own-traffic transmission, the cooperating node transmits its own packet to the destination using $\delta_{2}$ amount of energy. While in the relay transmission, the cooperating node forwards $N_{1}$ 's transmission to the destination using $\tilde{\delta}_{2}$ amount of energy. As in [53], we assume no packet/event transmission losses occurred. We also assume that sensors are deployed in a real-time monitoring scenario where no packets/events are buffered for later transmissions.

We assume that the cooperating node transmits its energy status $A$ to the source node as either 1 or 0 , representing whether the cooperating is energized or unenergized, respectively. 


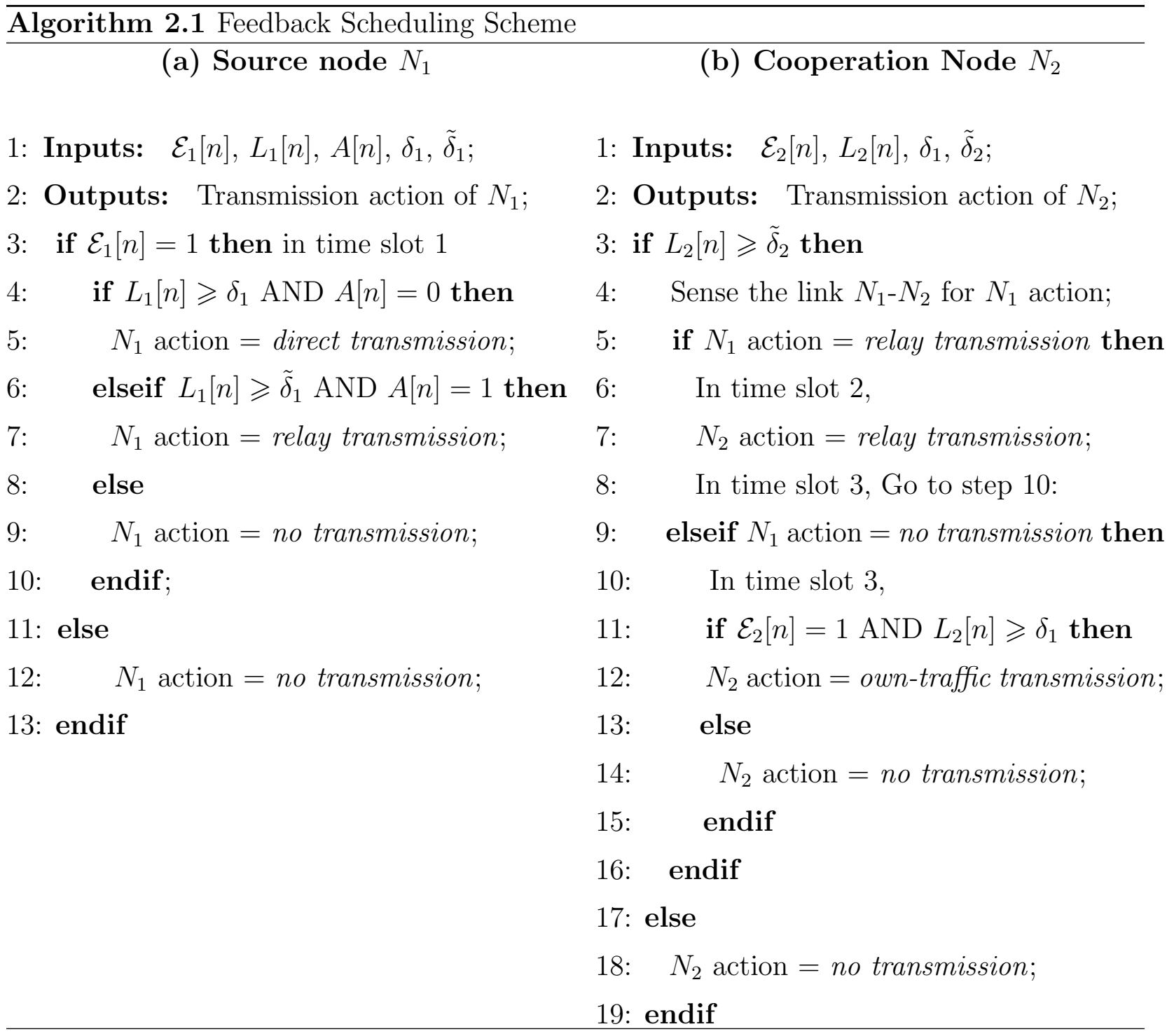

In the $n$-th time block, the cooperating node $N_{2}$ is said to be energized if it has sufficient energy to forward at least one event for the source, i.e, $L_{2}[n] \geqslant \tilde{\delta}_{2}$. Otherwise, it is said to be unenergized. We assume that the cooperating node status is transmitted only whenever it changes, i.e., if the cooperating node transmits its own packet or forwards the source packet and its status is still the same as before transmitting or forwarding, the cooperating node will not transmit its status. 


\subsection{Feedback Scheduling Scheme}

The feedback scheduling scheme proposed in this chapter is summarized in Algorithm 2.1. At the beginning of the time block $n \in\{1,2, \ldots, N\}$, the source node $N_{1}$ generates an event with probability modeled by the two-state process described earlier. And based on the event status $\mathcal{E}_{1}[n]$, the source in the first time slot takes one of the three transmission actions; direct transmission, relay transmission, or no transmission, as illustrated in part (a) of the algorithm. If $\mathcal{E}_{1}[n]=1$, the source takes direct transmission action if $A[n]=0$ and $L_{1}[n] \geqslant \delta_{1}$, i.e., if the cooperating node is unenergized and the source has sufficient energy to transmit directly. On the other hand, if $\mathcal{E}_{1}[n]=1$, the source takes relay transmission action if $A[n]=1$ and $L_{1}[n] \geqslant \tilde{\delta}_{1}$, i.e., if the cooperating node is energized and the source has sufficient energy to transmit cooperatively. In these two scenarios, the source's event is considered successfully delivered to the destination. If $\mathcal{E}_{1}[n]=1$ and the transmission action can neither be direct transmission nor relay transmission, the source takes the transmission action no transmission, and in this scenario the source's event is considered lost or not successfully reported to the destination. If $\mathcal{E}_{1}[n]=0$, the source takes the transmission action no transmission regardless of the energy availability at both the source and the cooperating node.

As for the cooperating node $N_{2}$, it takes a transmission action that is either own-traffic transmission, relay transmission, or no transmission, as illustrated in part (b) of the algorithm. In the first slot of the $n$-th time block, the cooperating node $N_{2}$ senses the $N_{1}-N_{2}$ link only if its latest energy status is energized. If there is a transmission coming from the source in that slot, which means that the transmission action taken at the source is a relay transmission, then the transmission action at the cooperating node will be taken as a relay transmission to forward the source's transmission in the second time slot. Otherwise, the transmission action will be taken as no transmission in the first two time slots. In the third time slot, the cooperating node takes the transmission action own-traffic to transmit its own event if $\mathcal{E}_{2}[n]=1$ and if $L_{2}[n] \geqslant \delta_{2}$. In these two scenarios, the event of the source and the event of the cooperating node are considered to be reported successfully to the destination. In the first two time slots, if the latest energy status of the cooperating node is unenergized, 
then the cooperating node will not sense the $N_{1}-N_{2}$ link since the transmission action at the source will not be taken as a relay transmission at that status. Hence, the transmission action will be taken as no transmission in the first two time slots. The transmission action will be taken as no transmission in the third time slot as well if the cooperating node has no event generated, or has no sufficient energy to transmit its event, i.e., $L_{2}[n]<\delta_{2}$. In this scenario, the cooperating node's event is considered lost. Note that in the algorithm, the default transmission action of each node is no transmission. If a node takes a transmission action that is not a no transmission in some time slot, the node will set its transmission action back to no transmission at the end of that time slot.

The feedback scheme is a distributed algorithm because each node performs some analysis and takes a decision on its transmission action by itself. As we will see later in the simulation results, the feedback algorithm maximizes the overall packet delivery ratio of the system, which is the ratio of the number of events that are successfully delivered for both the source and the cooperating node over the total number of events that are generated for both the source and the cooperating node over the $N$ time blocks. In the algorithm, however, the cooperating node prioritizes the source's transmission over its own, i.e., when both the source and the cooperating nodes have an event generated in the same time block, the cooperating node cooperates with the source first and then it transmits its own event if sufficient energy remained. If no sufficient energy remained, the cooperating node's event would be dropped. Consequently, the packet delivery ratio of the source will improve at the expense of the cooperating node. The problem can be reversed if the source priority condition $(A[n]=1$ if $\left.L_{2}[n] \geqslant \tilde{\delta}_{2}\right)$ is changed to the cooperating node priority condition $\left(A[n]=1\right.$ if $L_{2}[n] \geqslant \tilde{\delta}_{2}$ $\left.\& \mathcal{E}_{2}[n]=0\right)$. Under the cooperating node priority condition, the cooperating node will prioritize its transmission over the source's transmission which will improve its performance. Note that, the packet delivery ratio of a node is the ratio of the total events that are successfully delivered for that node to the total number of its generated events over the $N$ time blocks.

The flowcharts in Figure 2.3 illustrate the feedback and the threshold-based schemes. It can easily be noticed that the difference between them is only in the condition where the cooperating node is decided whether to be used or not. Our scheme bases its decision on 


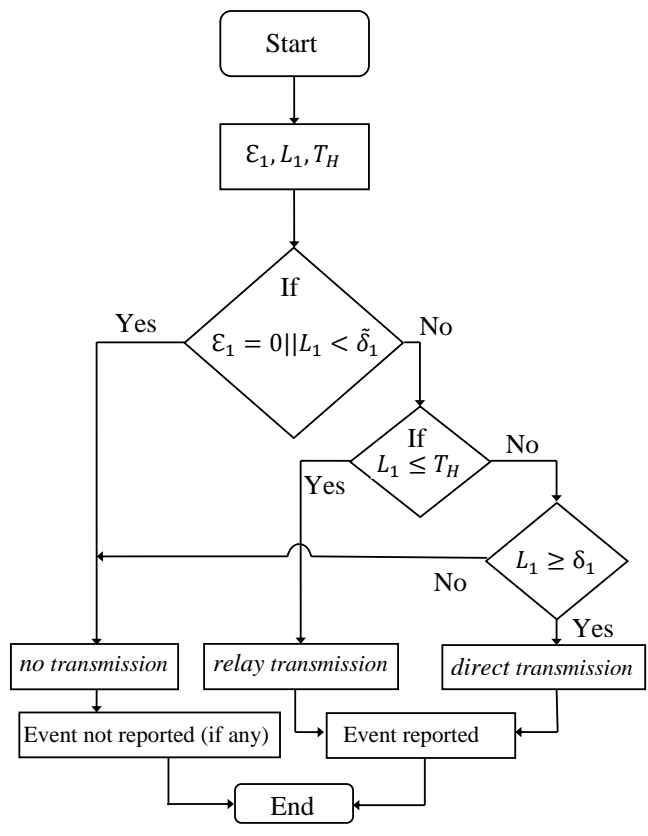

(a) Threshold-based scheme

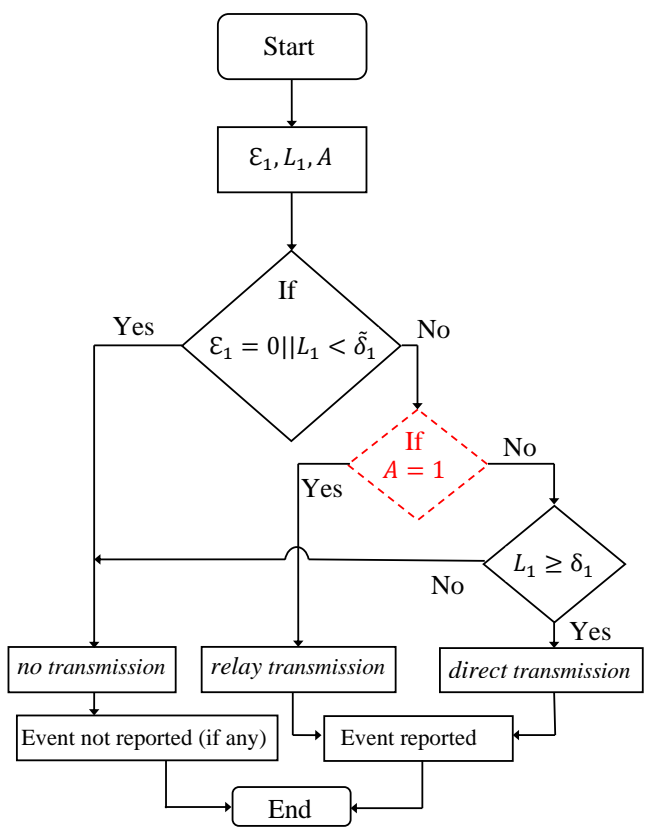

(b) Feedback scheme

Figure 2.3: Two flowcharts illustrate the feedback and the threshold-based schemes to easily notice the difference between them.

the actual energy status of the cooperating node while the threshold-based scheme bases its decision on the threshold value $T_{H}$. As stated before, the threshold value needs to be tuned for any given set of system parameters in order to maximize the system performance, and tuning it requires simulating the system at different energy battery levels of the source node, which is impractical in networks of unstable parameters or in networks of sensors with batteries of huge capacity.

\subsection{Simulation Results}

In this section, we evaluate the performance of the feedback scheme by comparing it with the performance of the threshold-based scheme. We simulate the two schemes using the same set of parameters that was used to simulate the threshold-based scheme in [53]. We simulate the feedback scheme for the two priority conditions to evaluate the performance of each node under each condition. In addition, we simulate the two schemes when one of the system parameters changes in order to show the sensitivity of the threshold-based scheme to 


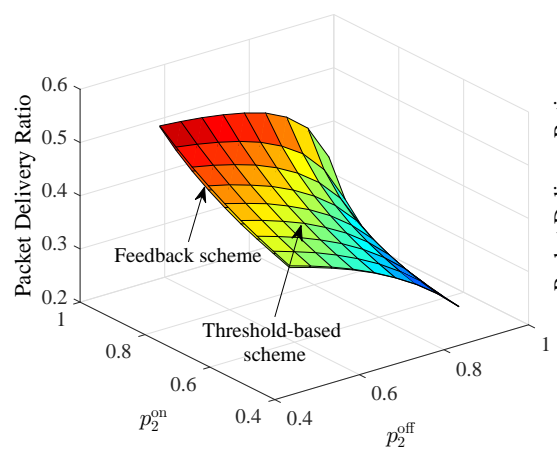

(a) Overall

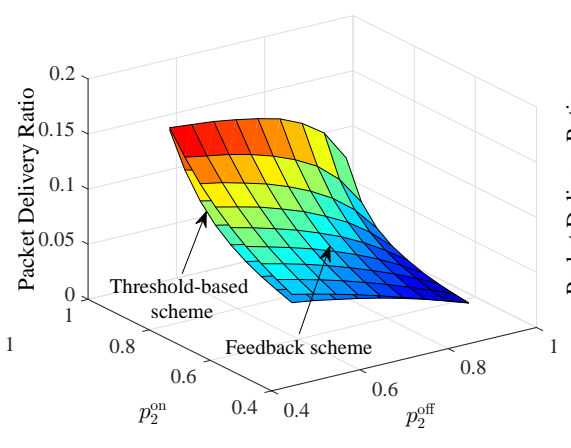

(b) Cooperating node

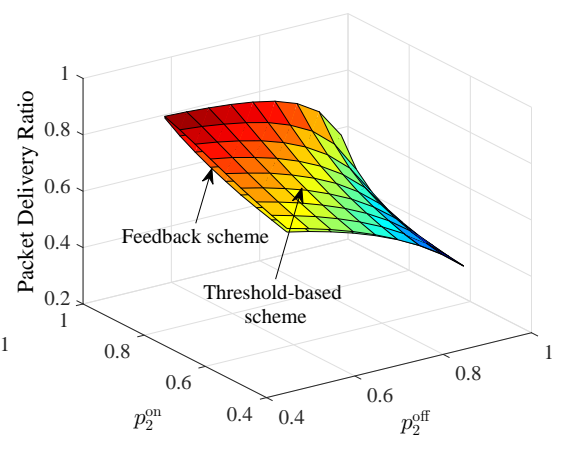

(c) Source

Figure 2.4: The overall, the cooperating node, and the source packet delivery ratios of the feedback and the threshold-based schemes. Parameters used: $q_{1}^{\text {on }}=0.9, q_{1}^{\text {off }}=0.7$, $p_{1}^{\text {on }}=q_{2}^{\text {on }}=0.85, p_{1}^{\text {off }}=q_{2}^{\text {off }}=0.7, E_{1}=E_{2}=1, \delta_{1}=\delta_{2}=3, \tilde{\delta}_{1}=\tilde{\delta}_{2}=1, K=20, T_{H}=10$.

the system parameters, and how our scheme stands in this case. The simulations were done using Matlab and they were run for a duration of $N=5 \times 10^{6}$ time blocks.

The performance is represented in terms of the packet delivery ratio versus energy harvesting probabilities of the cooperating node $p_{2}^{\text {on }}$ and $p_{2}^{\text {off }}$. Therefore, in the simulations, these two parameters are changing from 0.55 to 0.95 while the rest of the parameters are fixed. As in [53], the threshold value in the threshold-based scheme is set to the half of the battery capacity, i.e., $T_{H}=10$, which is the tunable value for the system parameters given in Figure 2.4 that maximizes the performance. The energy harvesting rate for both the source and the cooperating node $E_{1}$ and $E_{2}$ are set to 1 , where the source and the cooperating node each harvests 1 unit of energy in a time block if its energy harvesting state is "on" at that block. All the used parameters are given in the caption of the figures.

\subsubsection{Performance Evaluation}

Figure 2.4 illustrates the simulation results for the feedback and threshold-based schemes using the system parameters shown in the figure. The figure shows that the overall, the cooperating node, and the source packet delivery ratios for the two schemes are almost the same. In [53], it has been shown that the threshold-based scheme performs close to the optimum policy, meaning that, the feedback scheme performs also close to the optimum 


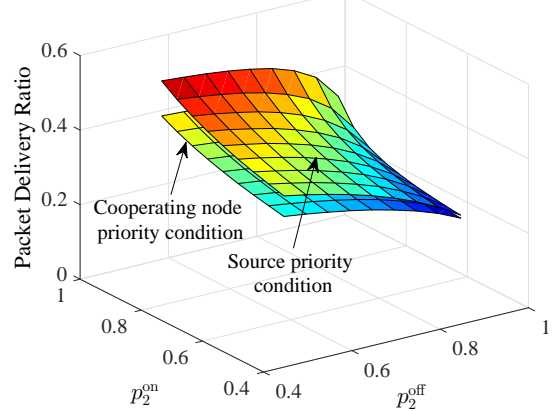

(a) Overall

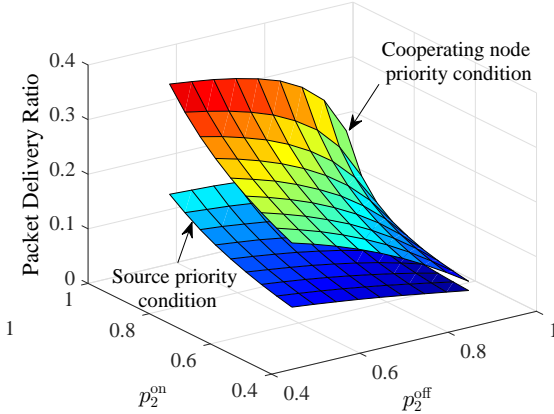

(b) Cooperating node

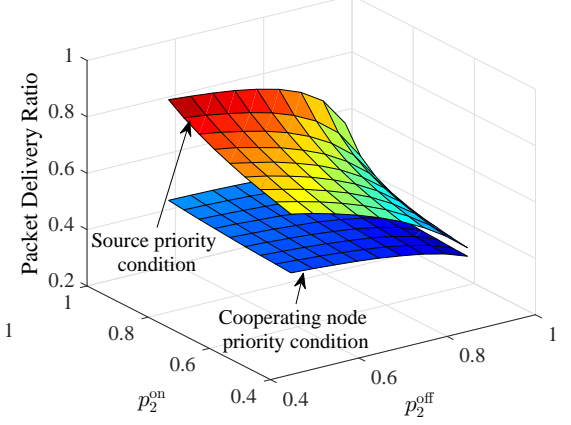

(c) Source

Figure 2.5: The overall, the cooperating node, and the source packet delivery ratios of the feedback scheme with both the source and the cooperating node priority conditions. Parameters used: $q_{1}^{\text {on }}=0.9, q_{1}^{\text {off }}=0.7, p_{1}^{\text {on }}=q_{2}^{\text {on }}=0.85, p_{1}^{\text {off }}=q_{2}^{\text {off }}=0.7, E_{1}=E_{2}=1$, $\delta_{1}=\delta_{2}=3, \tilde{\delta}_{1}=\tilde{\delta}_{2}=1, K=20, T_{H}=10$.

policy. However, the close performance to the optimum in the feedback scheme is achieved without requiring the optimization of system parameters.

From Figures 2.4 (a) and (b), it can be observed that the packet delivery ratio of the cooperating node is significantly lower than the source. In the two schemes, the cooperating node gives priority to the source's transmission over its own. Thus, the cooperating node spends most of its harvested energy on forwarding the source's packets instead of transmitting its own, which affects its packet delivery ratio. As we will see in the next simulation, the packet delivery ratio of the cooperating node can be improved in the feedback scheme by using the cooperating node priority condition mentioned in Section 2.3.

\subsubsection{Cooperating Node Priority Condition}

In this simulation, we simulate the feedback scheme using the cooperating node priority condition $\left(A=1\right.$ if $\left.L_{2} \geqslant \tilde{\delta}_{2} \& \mathcal{E}_{2}=0\right)$ and compare it with the case when the source priority condition $\left(A=1\right.$ if $L_{2} \geqslant \tilde{\delta}_{2}$ ) is used. Figure 2.5 (b) shows that the packet delivery ratio of the cooperating node improves when using the cooperating node priority condition compared to the source priority condition. However, the overall and the source packet delivery ratios decrease as can be noticed from Figures 2.5 (a) and (c). The packet delivery 


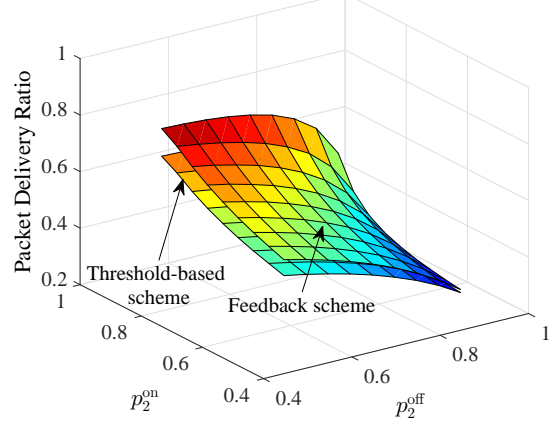

(a) Overall

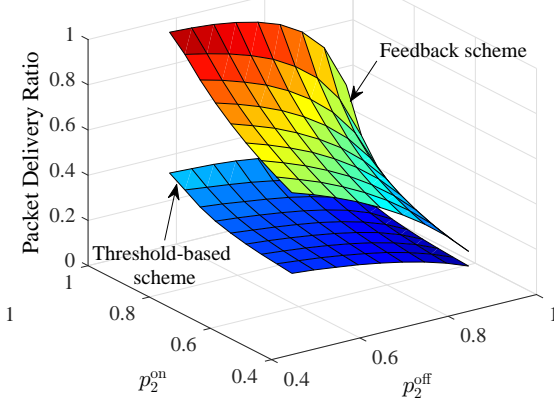

(b) Cooperating node

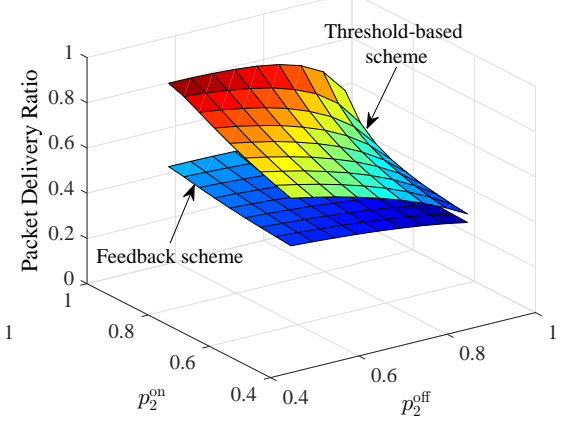

(c) Source

Figure 2.6: The overall, the cooperating node, and the source packet delivery ratios of the feedback and the threshold-based schemes when changing one of the parameters. Parameters used: $q_{1}^{\text {on }}=0.9, q_{1}^{\text {off }}=0.7, p_{1}^{\text {on }}=q_{2}^{\text {on }}=0.85, p_{1}^{\text {off }}=q_{2}^{\text {off }}=0.7, E_{1}=E_{2}=1, \delta_{1}=3$, $\delta_{2}=\tilde{\delta}_{1}=\tilde{\delta}_{2}=1, K=20, T_{H}=10$.

ratio of the source decreases because the cooperating node cooperates less with it when using the cooperating node priority condition. From Figures 2.5 (b) and (c), we can notice that the gap between the cooperating node and the source node packet delivery ratios decreases when using the cooperating node priority condition, which is more fair than when using the source priority condition.

\subsubsection{Threshold-based Scheme Sensitivity}

To show its sensitivity to the system parameters, we simulate the threshold-based scheme when $\delta_{2}$ changes from 3 to 1 while the threshold value $T_{H}$ is still the same, not tuned. We compare its performance with the feedback scheme. The simulation results in Figure 2.6 (a) show that the overall packet delivery ratio of the feedback scheme is better than the threshold-based scheme. This is because the performance in the threshold-based scheme is not maximized due to the fact that the threshold value $T_{H}=10$ is not the tunable value for the new set of parameters. Hence, we conclude that using the threshold-based scheme in networks of unstable parameters is impractical when the maximum packet delivery ratio of the system needs to be maintained. Finally, Figure 2.6 (c) illustrates that the packet delivery ratio of the source in the threshold-based scheme is better than the feedback scheme. This is 
because in the threshold-based scheme, the cooperating node in this new set of parameters cooperates more with the source, which results in better packet delivery ratio for the source, and worse packet delivery ratio for the cooperating node as can be seen from Figure 2.6 (b).

\subsection{Conclusions}

This chapter considers an energy harvesting three-node network of a source, a cooperating node, and a destination, and it proposes a simple practical scheme, called feedback scheme, that maximizes the packet delivery ratio of the system. The feedback scheme can be generalized to any network of many sensor nodes when each group of three nodes in the network can form a three-node relay channel. In the opposite to the threshold-based scheme, the feedback scheme does not require auxiliary parameter optimization in order to maximize the system performance. This advantage makes the feedback scheme easier and more practical to be used, especially in networks with unstable parameters or with sensors of huge battery capacity.

The feedback scheme can easily switch between two different conditions on the cooperating node energy status, cooperating node priority condition and source priority condition, in order to prioritize the transmissions of either the source or the cooperating node. Hence, if the energy harvesting rate of the cooperating node is higher than its event occurrence rate and the opposite holds true for the source, then the feedback scheme should use the source priority condition. Thus, the cooperating node cooperates more and more events will be delivered for the source. While if the energy harvesting rate of the cooperating node is lower than its event occurrence rate and the opposite holds true for the source, the feedback scheme should use the cooperating node priority condition. Thus, the cooperating node cooperates less and more events will be delivered for the cooperating node. The switch between these two conditions can easily be done by the cooperating node, assuming that the event occurrence probability and the energy harvesting rate of the source are known at the cooperating node. In the threshold based scheme, prioritizing the transmissions of either the source or the cooperating node is not easy. It requires tuning the threshold level at the optimal value, which requires simulating the system at different levels of the source's energy 
battery. Lastly, the feedback scheme is distributed as each node performs some analysis and makes a decision on its transmission action by itself. 


\section{Chapter 3}

\section{Multiple Cooperating Nodes Scheduling}

\subsection{Introduction}

In this chapter, the feedback scheme is upgraded to include the scenario of multiple cooperating nodes in one-way or two-way cooperative communications. In this scenario, we assume that the system has multiple cooperating nodes that assist cooperative communication between two transmitting nodes, as well as they transmit their own events to one of the transmitting nodes in the network. In the algorithm, the cooperating nodes are indexed increasingly, and each node is assumed to broadcast its energy status to the network as either energized or unenergized. When any of the two transmitting nodes has a transmission and there is more than one energized cooperating node, then the transmitting node uses the cooperating node of the highest index. In another scenario, the transmitting node uses the cooperating nodes sequentially in order to be fair between the cooperating nodes. Using a cooperating node definitely improves the performance of the transmitting nodes. However, it increases the power consumed by the cooperating node, which affects its packet delivery ratio. Therefore, we propose an adaptive scheduling algorithm that incorporates fairness constraints to ensure the same performance between the nodes.

Portion of this chapter appears in: A. Ammar and D. Reynolds, "An adaptive scheduling scheme for cooperative energy harvesting networks," Journal of Communications and Networks, vol.17, no.3, pp.256-264, June 2015. 


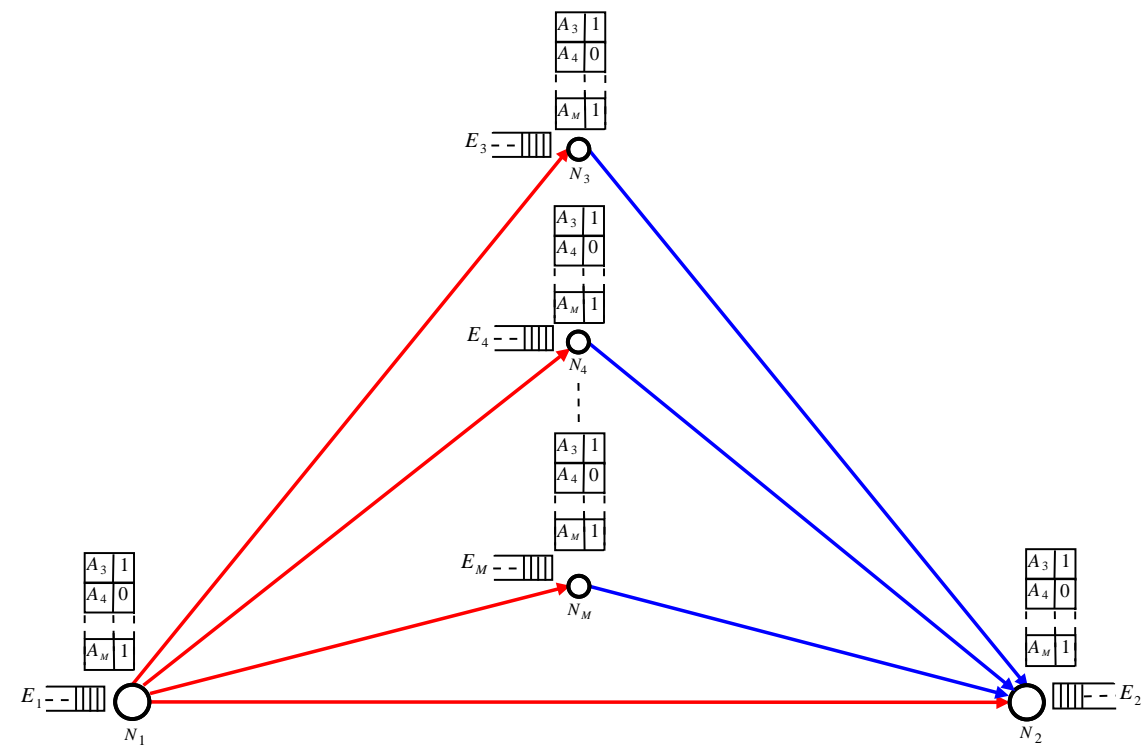

Figure 3.1: Energy harvesting sensor network of $M-2$ cooperating nodes that assist cooperative communication between two sensors $\left(N_{1}, N_{2}\right)$. Each node $N_{m}, m \in\{1,2, \ldots, M\}$ harvests $E_{m}$ amount of energy if its energy generation process is "on". Each node has a memory of $M-2$ slots to save the latest status, $A_{m}, m \in\{3,4, \ldots, M\}$, of the cooperating node $N_{m}$ as either 1 or 0 in the corresponding $m$-th slot.

\subsection{System Model}

We consider a wireless sensor network with $M$ sensor nodes, including $M-2$ cooperating nodes that assist cooperative communications between two nodes $\left(N_{1}\right.$ and $\left.N_{2}\right)$, as depicted in Figure 3.1. We allow for one-way or two-way cooperative scenarios. In the one-way scenario, $N_{1}$ and $N_{2}$ serve as a source and destination, respectively, and each cooperating node $N_{m}, m \in\{3,4, \ldots, M\}$ serves as a relay for $N_{1}$ and as a source to transmit its own packets to the destination. In the two-way scenario, $N_{1}$ and $N_{2}$ serve as both a source and a destination, and each cooperating node $N_{m}$ serves as a source and also as a relay for both $N_{1}$ and $N_{2}$. Each sensor node is equipped with energy harvesting devices and has a rechargeable battery with capacity $K$. The energy available at node $N_{m}, m \in\{1,2, \ldots, M\}$, is $L_{m} \in\{0,1,2, \ldots, K\}$. As in the previous chapter, we assume no errors or packet losses occurred and that sensors are deployed in real-time monitoring scenarios where no packets are buffered. Based on these assumptions, there is no need to use more than one cooperating 
node per transmission.

As in the previous chapter, we assume a discrete-time model, where time is divided into blocks. We also use a TDMA protocol to avoid collision, however, here each block is divided into a number of time slots that depends on the scenario. In the one-way scenario, there are $M$ time slots where the first slot is assigned for node $N_{1}$, the second is assigned for a cooperating node to forward $N_{1}$ 's transmission, and the rest are assigned for the cooperating nodes sequentially for their own transmissions. In the two-way scenario, there is $M+2$ time slots, i.e., two extra time slots. One of these extra slots is the third time slot, which is assigned for $N_{2}$, and the second extra slot is the fourth time slot, which is used for forwarding $N_{2}$ 's transmission.

During each time slot in which there is an event to report, the sensor node $N_{i}, i \in\{1,2\}$ operates in one of two transmission modes; direct transmission or relay transmission. In direct transmission, the sensor node $N_{i}$ transmits its packet directly to the destination using $\delta_{i}$ amount of energy. In relay transmission, the sensor node $N_{i}$ transmits its packet to the destination using $\tilde{\delta}_{i}$ amount of energy, and it also makes use of a cooperating node $N_{m}$, $m \in\{3, \ldots, M\}$ to forward its transmission to the destination in the next time slot. The cooperating node $N_{m}$ also operates in one of two transmission modes; own-traffic transmission or relay transmission. In the own-traffic transmission, the cooperating node transmits its own packet to the destination using $\delta_{m}$ amount of energy. While in the relay transmission, the cooperating node forwards the event of node $N_{i}$ to the destination using $\tilde{\delta}_{m}$ amount of energy.

As in Chapter 2, we assume that there is at most one event generated per transmitting sensor per block. Both the event generation and the energy harvesting processes at each node are modeled by a temporally correlated, two-state Markov process ("on", "off"). For node $N_{i}, i \in\{1,2, \ldots, M\}$, the transition probabilities of the event generation process are $q_{i}^{\text {on }}$ and $q_{i}^{\text {off }}$ while the transition probabilities of the energy generation process are $p_{i}^{\text {on }}$ and $p_{i}^{\text {off }}$. The event generation status in the $n$-th time block for node $N_{i}$ is denoted by $\mathcal{E}_{i}[n] \in\{1,2\}$, and the amount of harvested energy in a time block when the state is "on" is denoted by $E_{i}$.

The cooperating node $N_{m}, m \in\{3,4, \ldots, M\}$ is assumed to broadcast its energy status $A_{m} \in\{0,1\}$ to the network whenever it changes, and all the $M$ nodes are assumed to have 
a memory of $M-2$ slots, indexed as $S_{3}, S_{4}, \ldots, S_{M}$, where the most recent status of each cooperating node is stored. As stated in Chapter 2, the status of 1 corresponds to the cooperating node status energized, and the status of 0 corresponds to the cooperating node status unenergized. In the $n$-th time block, the cooperating node $N_{m}$ is said to be energized if it has sufficient energy to forward at least one event, i.e., it satisfies $L_{m}[n] \geqslant \tilde{\delta}_{m}$. Otherwise, it is said to be unenergized. In the $n$-th time block, we assume that $\mathcal{A}[n]$ is a set that includes all the energized cooperating nodes.

Broadcasting a cooperating node status whenever it changes increases the overhead of the energy statuses' transmissions, which could consume a significant amount of energy and increase the computational complexity modestly (linearly) with the number of cooperating nodes. This burden can be reduced when $p_{m}^{\text {on }}$, the energy harvesting probability of the cooperating node $N_{m}$, is low by adapting the energy status broadcasting of that node. Such that, if the cooperating node $N_{m}$ transmits, then all the $M$ sensors will automatically set the energy status of that node to unenergized. This is because node $N_{m}$ is more likely to be unenergized after transmitting due to its low rate of energy harvesting. If node $N_{m}$ is still energized after transmitting, the node will broadcast 1 to the network so that all the nodes set its status back to energized.

\subsection{Scheduling Algorithm}

Algorithm 3.1 describes the scheduling algorithm for the one-way scenario. As illustrated in part (a) of the algorithm, in the $n$-th time block, $n \in\{1,2, \ldots, N\}$, node $N_{1}$ generates an event $\mathcal{E}_{1}$ using the Markov process described earlier. If $\mathcal{E}_{1}[n]=1$, then the transmission action will be direct transmission if all the cooperating nodes are unenergized and $L_{1}[n] \geqslant$ $\delta_{1}$. If $\mathcal{E}_{1}[n]=1$, then the transmission action will be relay transmission if there is at least one energized cooperating node and $L_{1}[n] \geqslant \tilde{\delta}_{1}$. In these two scenarios, the event of $N_{1}$ is considered successfully reported. If $\mathcal{E}_{1}[n]=1$ and the transmission action can neither be direct transmission nor relay transmission, then the transmission action will be no transmission. In this scenario, the event of $N_{1}$ is considered not successfully reported or lost. If $\mathcal{E}_{1}[n]=0$, then the transmission action will also be no transmission. When 


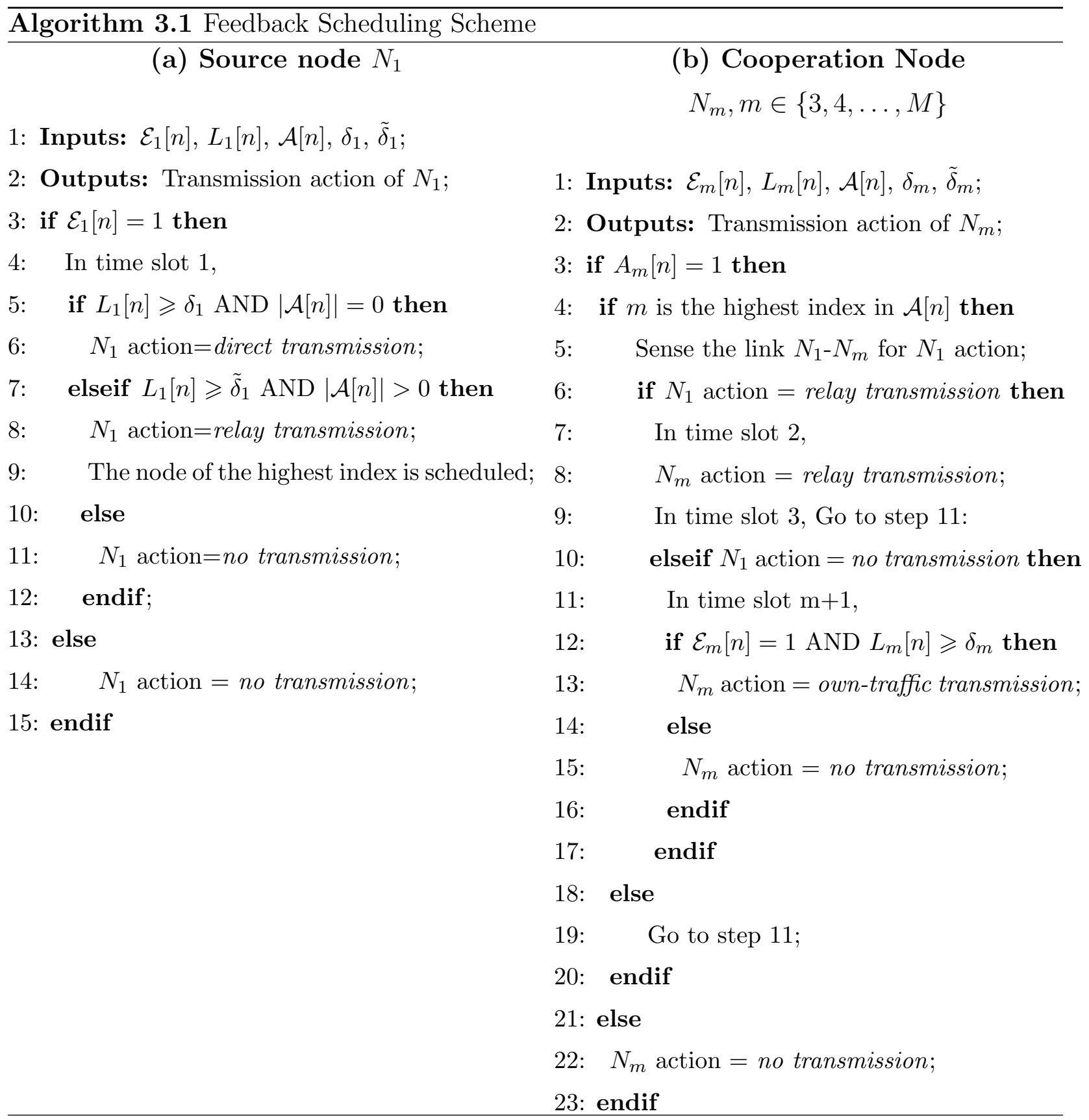




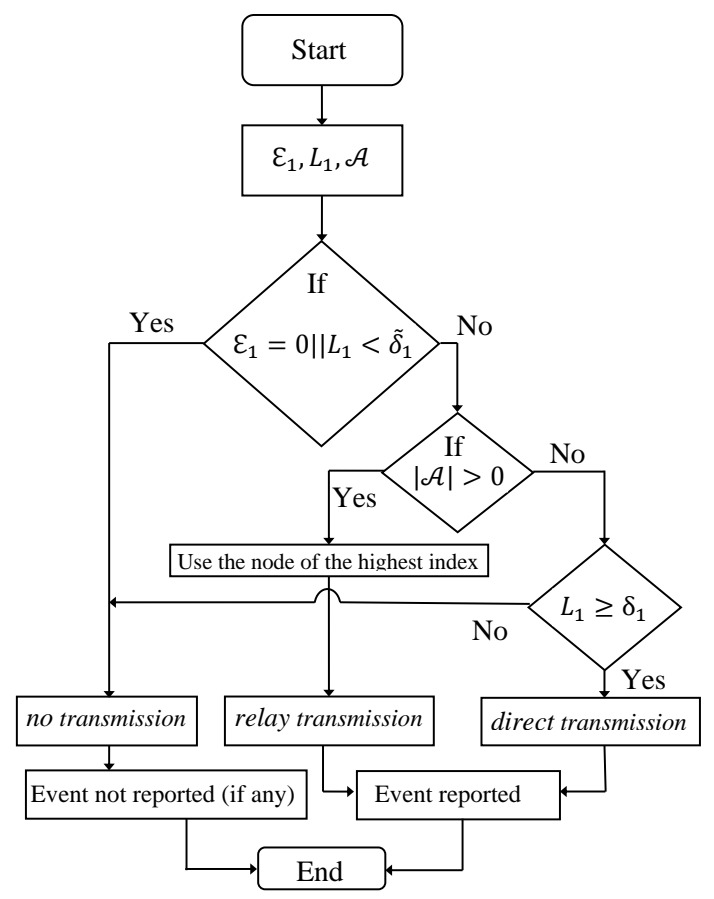

Figure 3.2: A flowchart illustrates the feedback scheme for the case of multiple cooperating nodes when the cooperating node of the highest index is selected for relay transmission.

the transmission action is relay transmission, there is no advantage to using more than one cooperating node because we assume no errors or packet losses occurred. If there is more than one energized cooperating node, the algorithm will arbitrarily choose the cooperating node with the highest index number. Note that, choosing the cooperating node with the highest index causes the cooperating node of the highest index to be scheduled more than the others, which is unfair. This problem could be solved, for example, by choosing the cooperating nodes sequentially instead of always choosing the cooperating node of the highest index, i.e., replace line 9 of part (a) of Algorithm 3.1 with "Schedule the cooperating nodes sequentially". However, scheduling the cooperating nodes sequentially may require the cooperating nodes to track the most recently used cooperating node to decide which cooperating node should cooperate next. The flowchart in Figure 3.2 illustrates how the decision is taken at the source node $N_{1}$ given the set of the energized cooperating nodes $\mathcal{A}[n]$ as well as its current energy battery level and event status.

With regard to the cooperating nodes, in the first time slot of the block, the energized cooperating node with the highest index number, say node $N_{m}, m \in\{3,4, \ldots, M\}$, will sense 
the $N_{1}-N_{m}$ link. If $N_{1}$ has transmitted, this means that the transmission action taken at $N_{1}$ is a relay transmission. Therefore, the transmission action at that cooperating node will be taken as a relay transmission to forward $N_{1}$ 's transmission in the next time slot. If not, the cooperating node will take no action and there will be no transmission in the first two slots. In the rest of the time slots, each cooperating node generates an event $\mathcal{E}_{m}$ with independent realizations of the same Markov process, and it takes a transmission action in its corresponding time slot based on its $\mathcal{E}_{m}[n]$ and $L_{m}[n]$. If $\mathcal{E}_{m}[n]=0$ or $L_{m}[n]<\delta_{m}$, then the transmission action will be taken as no transmission. If $\mathcal{E}_{m}[n]=1$ and $L_{m}[n] \geqslant \delta_{m}$, then the transmission action will be taken as an own-traffic transmission, and the event will be reported successfully. If $\mathcal{E}_{m}[n]=1$ and $L_{m}[n]<\delta_{m}$, the transmission action will be taken as a no transmission, and the event will be dropped.

For the two-way scenario, node $N_{2}$ can use part (a) of the algorithm to schedule one of the cooperating nodes if slot 1 in line 4 is changed to slot 3 . And the cooperating node of the highest index that will cooperate with $N_{2}$ can use part (b) of the algorithm if slot 2 in line 7 is changed to slot 4 , slot 3 in line 9 is changed to slot 5 , and slot $\mathrm{m}+1$ is changed to slot $\mathrm{m}+2$. As in Algorithm 2.1, the default transmission action of each node is no transmission. Thus, if a node takes a transmission action that is not a no transmission in some time slot, the node will set its transmission action back to no transmission at the end of that time slot.

\subsection{Adaptive Scheme for Absolute Fairness}

In Chapter 2, we saw that the feedback scheme can easily switch between the two conditions, source priority condition and cooperating node priority condition, in order to prioritize the transmissions of either the source or the cooperating node. Hence, the packet delivery ratio of the prioritized node over the $N$ time blocks becomes higher than the other, which may not be desired in some applications when the same performance needs to be achieved by the nodes. Therefore, we here propose an adaptive scheme that automatically switches between the two priority conditions such that the nodes achieve the same performance over the $N$ time blocks. Again, for the cooperating node $N_{m}, m \in\{3,4, \ldots, M\}$, the source prior- 
ity condition is $\left(A_{m}[n]=1\right.$ if $\left.L_{m}[n] \geqslant \tilde{\delta}_{m}\right)$, which prioritizes the transmissions of $N_{1}$ and $N_{2}$, and the cooperating node priority condition is $\left(A_{m}[n]=1\right.$ if $L_{m}[n] \geqslant \tilde{\delta}_{m}$ and $\left.E_{m}[n]=0\right)$, which prioritizes the cooperating nodes' transmissions. The algorithm switches adaptively between these two conditions based on the current relative performance of $N_{1}, N_{2}$, and the cooperating nodes. More specifically, we assume that the packet delivery ratio of, say $N_{1}$, is sent to the cooperating nodes after each interval of $T$ blocks. In the first interval, the decision on the cooperating nodes' energy status is made by the source priority condition for the first half of the blocks, and by the cooperating node priority condition for the second half. After the first interval, the cooperating nodes will change the number of blocks of each condition after receiving the packet delivery ratio of $N_{1}$. If the packet delivery ratio of $N_{1}$ is higher than the cooperating nodes, the cooperating nodes will increase the number of blocks of the cooperating node priority condition at the expense of the source priority condition time blocks, so that the cooperating nodes cooperate less with $N_{1}$ in the next interval which improves their performance. In the case of the opposite, however, the cooperating nodes will increase the number of the time blocks of the source priority condition at the expense of the cooperating node priority condition time blocks, so that the cooperating nodes cooperate more with $N_{1}$ in the next interval which improves $N_{1}$ 's performance. Changing the number of time blocks of the two conditions is done gradually, with a step size that can be chosen depending upon the divergence gap between $N_{1}$ and the cooperating nodes' packet delivery ratios and $T$.

As we will see in Section 3.5.4.2, the system will converge and ensure fairness for a fixed set of parameters. However, changing any of the parameters relatively changes the packet delivery ratio of $N_{1}$ and the cooperating nodes. Therefore, after converging, if any of the parameters changes, the system will diverge and the fairness problem will occur again. This indicates that changing any of the system parameters influences fairness, and as we will see in Section 3.5.4.2, the system will adapt as parameters change. With respect to the threshold-based scheme and to the best of our knowledge, there is no straightforward way to adapt the scheme for fairness. 


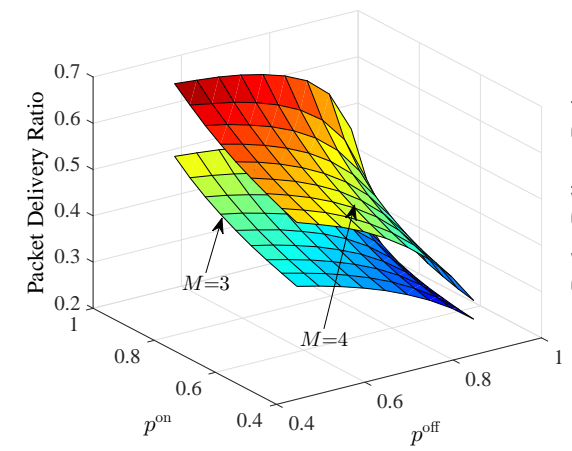

(a) Overall packet delivery ratio

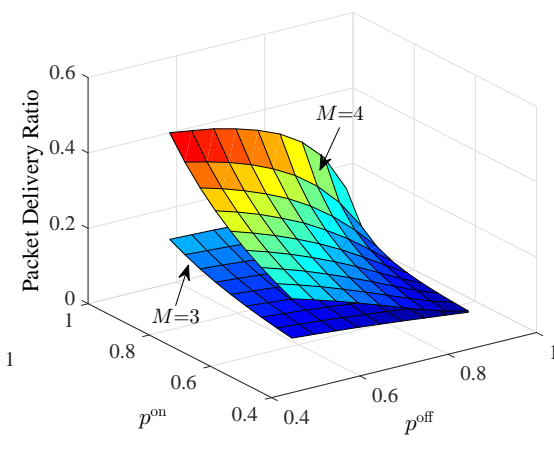

(b) $N_{3}$ packet delivery ratio

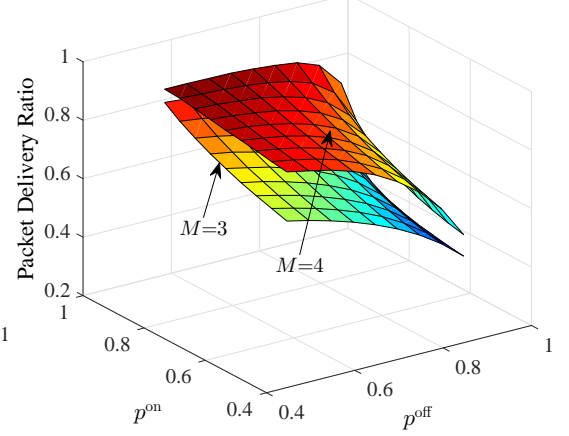

(c) $N_{1}$ packet delivery ratio

Figure 3.3: The packet delivery ratios of the one-way scenario when $M=3$ and $M=4$. Parameters used: $q_{1}^{\text {on }}=0.9, q_{1}^{\text {off }}=0.7, p_{1}^{\text {on }}=q_{3}^{\text {on }}=0.85, p_{1}^{\text {off }}=q_{3}^{\text {off }}=0.7, E_{1}=E_{3}=1$, $\delta_{1}=\delta_{3}=3, \tilde{\delta}_{1}=\tilde{\delta}_{3}=1, K=20$.

\subsection{Simulation results}

We simulate the proposed scheme in Section 3.3 for one-way and two-way scenarios when $M=3$ and $M=4$. We use the same set of parameters used in the previous chapter, which is shown in the figures. We also simulate the adaptive way of broadcasting the energy status described at the end of Section 3.2. Moreover, we simulate the adaptive scheduling scheme described in Section 3.4. In the simulations, the event occurrence parameters $\left(q_{i}^{\text {on }}\right.$, $\left.q_{i}^{\text {off }}\right)$ for $N_{i}, i \in\{1,2, \ldots, M\}$, and the energy harvesting process parameters $\left(p_{i}^{\text {on }}, p_{i}^{\text {off }}\right)$ for $N_{i}, i \in\{1,2\}$ are fixed while the energy harvesting process parameters $\left(p_{m}^{\text {on }}, p_{m}^{\text {off }}\right)$ for $N_{m}, m \in$ $\{3,4, \ldots, M\}$ are allowed to vary. We set $q_{i}^{\text {on }}, q_{i}^{\text {off }}, p_{i}^{\text {on }}$, and $p_{i}^{\text {off }}$ to be the same for all $i \in\{1,2\}$, and we also set $q_{m}^{\text {on }}$ and $q_{m}^{\text {off }}$ to be the same for all $m \in\{3,4, \ldots, M\}$. In order to plot the results, we set $p_{m}^{\text {on }}=p^{\text {on }}$ and $p_{m}^{\text {off }}=p^{\text {off }}$ for all $m \in\{3,4, \ldots, M\}$, and they both vary from 0.55 to 0.95 .

\subsubsection{One-Way Cooperative Scenario}

As stated in Section 3.2, in the one-way scenario node $N_{1}$ operates as a source while node $N_{2}$ operates as a destination, and the remaining $M-2$ nodes operate as cooperating nodes to relay for $N_{1}$ and as sources to transmit their own events to $N_{2}$. So, in this scenario, all the transmitted packets will flow only in one direction, from $N_{1}$ and $\left\{N_{m}\right\}_{m=3}^{M}$ towards $N_{2}$. 


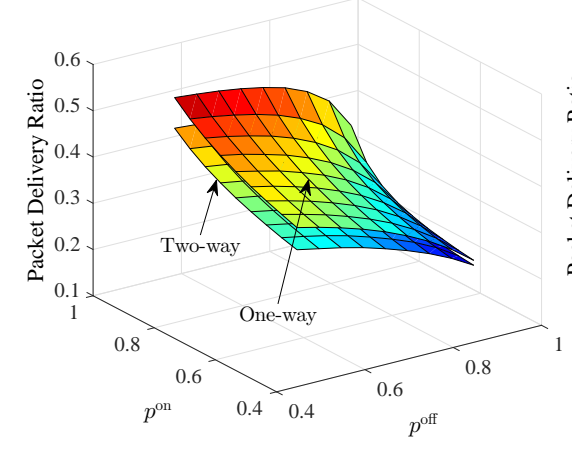

(a) Overall packet delivery ratio

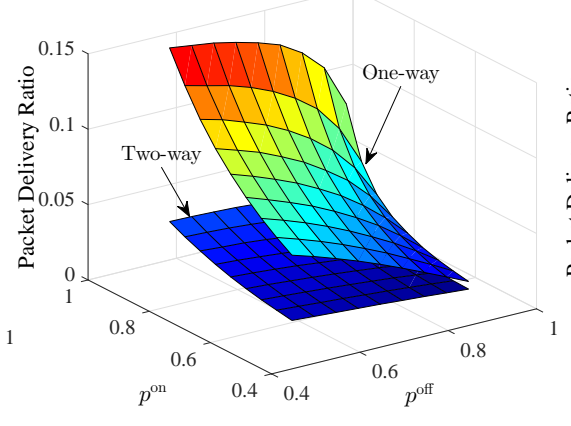

(b) $N_{3}$ packet delivery ratio

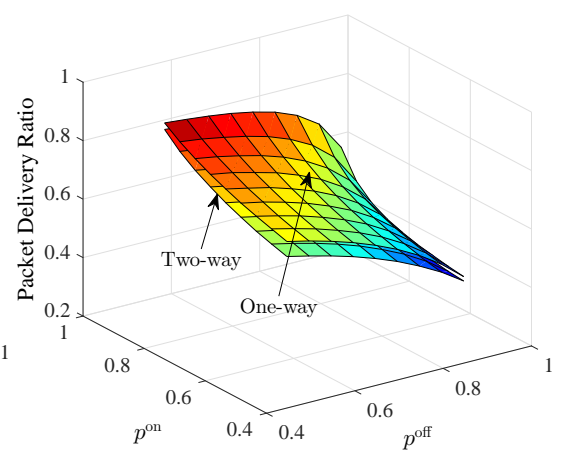

(c) $N_{1}$ packet delivery ratio

Figure 3.4: The packet delivery ratios of the one-way and two-way scenarios when $M=3$. Parameters used: $q_{1}^{\text {on }}=0.9, q_{1}^{\text {off }}=0.7, p_{1}^{\text {on }}=q_{3}^{\text {on }}=0.85, p_{1}^{\text {off }}=q_{3}^{\text {off }}=0.7, E_{1}=E_{3}=1$, $\delta_{1}=\delta_{3}=3, \tilde{\delta}_{1}=\tilde{\delta}_{3}=1, K=20$.

Based on $\left\{A_{m}\right\}_{m=3}^{M}, L_{1}$, and $\mathcal{E}_{1}, N_{1}$ schedules a cooperating node $N_{m}$ for usage in its time slot using Algorithm 3.1.

Using the parameters given in Figure 3.3, the scenario was simulated using Algorithm 3.1 for the case when the system has only one cooperating node $N_{3}$, i.e., $M=3$, and for the case when the system has two cooperating nodes $N_{3}$ and $N_{4}$, i.e., $M=4$. The simulation results in Figure 3.3 compare the performance of the two cases. To make a fair comparison, the overall performance in the case when $M=4$ was represented by the packet delivery ratio of $N_{1}$ and $N_{3}$. The results show that the performance of $N_{3}$ and the performance of $N_{1}$ improve when $M=4$. When $M=4, N_{1}$ has a better chance to transmit cooperatively than when $M=3$, so that more energy will be saved and more packets will be delivered for $N_{1}$. Also, when $M=4$, each cooperating node cooperates less with $N_{1}$ since the nodes share the cooperation between them. Less cooperation with $N_{1}$ means more packets delivered for $N_{3}$. Of course, increasing the number of delivered packets for $N_{1}$ and $N_{3}$ improves their performance, and the overall performance of the system.

\subsubsection{Two-Way Cooperative Scenario}

In the two-way scenario, $N_{1}$ and $N_{2}$ both operate as a source and destination, and the remaining $M-2$ nodes operate as cooperating nodes to relay for $N_{1}$ and $N_{2}$, and 


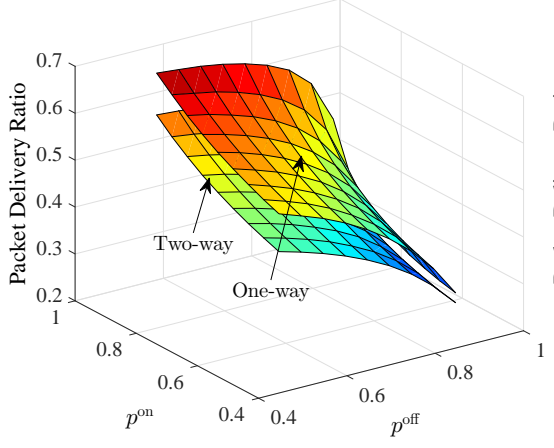

(a) Overall packet delivery ratio

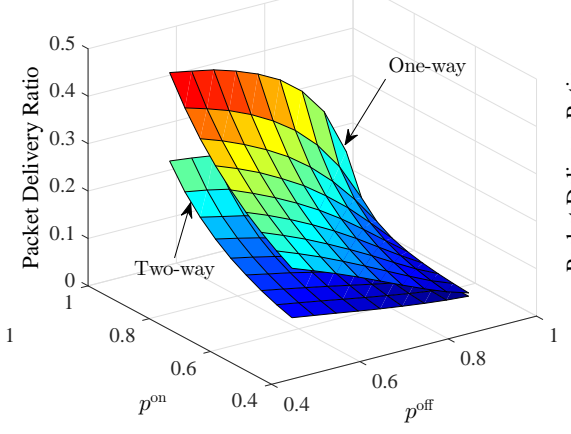

(b) $N_{3}$ packet delivery ratio

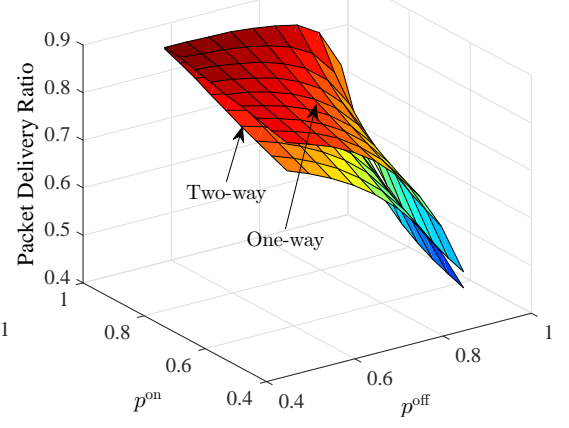

(c) $N_{1}$ packet delivery ratio

Figure 3.5: The packet delivery ratios of the one-way and two-way scenarios when $M=4$. Parameters used: $q_{1}^{\text {on }}=0.9, q_{1}^{\text {off }}=0.7, p_{1}^{\text {on }}=q_{3}^{\text {on }}=0.85, p_{1}^{\text {off }}=q_{3}^{\text {off }}=0.7, E_{1}=E_{3}=1$, $\delta_{1}=\delta_{3}=3, \tilde{\delta}_{1}=\tilde{\delta}_{3}=1, K=20$.

operate as sources as well. So, in this scenario, all the transmitted packets will flow in two directions, from $N_{1}$ and $\left\{N_{m}\right\}_{m=3}^{M}$ towards $N_{2}$, and from $N_{2}$ and $\left\{N_{m}\right\}_{m=3}^{M}$ towards $N_{1}$. Using Algorithm 3.1, $N_{1}$ and $N_{2}$ both schedule a node $N_{m}$ for cooperation using their parameters $L_{i}, \mathcal{E}_{i}, i \in\{1,2\}$, and the cooperating nodes energy statuses $\left\{A_{m}\right\}_{m=3}^{M}$ as described earlier.

The simulation results in Figure 3.4 illustrate the performance of the algorithm for the two-way scenario when $M=3$. The results also compare the performance of the two-way scenario with the one-way scenario when $M=3$. To make the overall performance of the two scenarios fairly comparable, the overall performance of the two-way scenario was represented by the packet delivery ratios of $N_{1}$ and $N_{3}$, meaning that the performance of $N_{2}$ was not included. The results show that the performance of the two-way scenario is worse than the one-way scenario. This is reasonable since $N_{3}$ cooperates with two nodes in the two-way scenario instead of one. Cooperating with two nodes increases the cooperation load on $N_{3}$ and thus affects its performance. Also, it decreases the cooperation chance of $N_{3}$ with $N_{1}$ which affects the performance of $N_{1}$. As a result, the overall performance of the system decreases due to the decrease in the performance of $N_{1}$ and $N_{3}$.

The algorithm was also simulated for the two-way scenario with two cooperating nodes $N_{3}$ and $N_{4}$, i.e., $M=4$. Figure 3.5 shows the simulation results for the two-way scenario compared with the one-way scenario when $M=4$. The overall performance of the two 


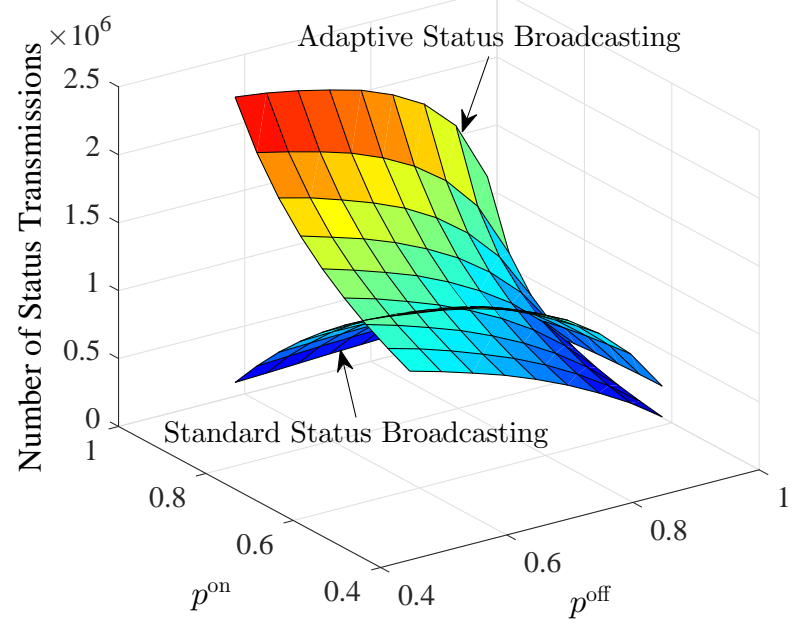

Figure 3.6: The number of status transmissions of the standard and adaptive status broadcastings for the one-way scenario when $M=3$. Parameters used: $q_{1}^{\text {on }}=0.9, q_{1}^{\text {off }}=0.7$, $p_{1}^{\text {on }}=q_{3}^{\text {on }}=0.85, p_{1}^{\text {off }}=q_{3}^{\text {off }}=0.7, E_{1}=E_{3}=1, \delta_{1}=\delta_{3}=3, \tilde{\delta}_{1}=\tilde{\delta}_{3}=1, K=20$.

scenarios is represented by the packet delivery ratio of $N_{1}$ and $N_{3}$.

\subsubsection{Overhead Reduction}

In Section 3.2, we mentioned that broadcasting a cooperating node status in the standard way (whenever it changes) increases status transmissions overhead, and we suggested an adaptive way of a cooperating node status broadcasting that could reduce this burden for low values of $p^{\text {on }}$. Here, we simulate the system when $M=3$ for the standard and adaptive ways of status broadcasting. Figure 3.6 illustrates the number of status transmissions of $N_{3}$ for the two ways. It can be noticed that the number of status transmissions is reduced when $p^{\text {on }} \leqslant 0.75$, where the energy harvesting probability of $N_{3}$ is considered low. Although this burden is reduced for some values of $p^{\text {on }}$, the burden is still relatively high. Therefore, in Chapter 5 we develop a statistical model that enables the source to estimate the energy status of the cooperating nodes. 


\subsubsection{Fairness}

\subsubsection{Between Cooperating Nodes}

In Algorithm 3.1, if there is more than one active cooperating node, the algorithm will arbitrarily select the node with the highest index number. As mentioned earlier in Section 3.3, this selection causes an unfairness problem between the cooperating nodes, and this problem could be solved by selecting the cooperating nodes sequentially instead of always selecting the cooperating node of the highest index. The algorithm was simulated for the two ways of selection for the one-way system when $M=4$. Figure 3.7 illustrates the simulation results, where Figure 3.7 (a) illustrates the overall packet delivery ratio which is represented by $N_{1}$ and $N_{3}$, Figure 3.7 (b) illustrates the packet delivery ratios of the cooperating nodes $N_{3}$ and $N_{4}$, and Figure 3.7 (c) illustrates the packet delivery ratio of node $N_{1}$.

It can be noticed from Figure 3.7 (b) that $N_{3}$ and $N_{4}$ have the same packet delivery ratio when the nodes are selected sequentially. This ensures fairness between the cooperating nodes in contrast to the highest node selection case where the packet delivery ratio of $N_{4}$ is less than $N_{3}$. Figure 3.7 (c) shows that the packet delivery ratio of $N_{1}$ is virtually the same for the two ways of selection. This is expected since the only difference between the two ways of selection is deciding which node should cooperate with $N_{1}$. Figure 3.7 (a) shows that the overall packet delivery ratio when the highest cooperating node is always selected is better than it when the cooperating nodes are selected sequentially. This is also expected since the cooperating node $N_{3}$ is used more often when the cooperating nodes are selected sequentially than when the highest cooperating node is always selected.

\subsubsection{Between Cooperating Nodes and $N_{1}$ and/or $N_{2}$}

The adaptive scheduling scheme described in Section 3.4 is simulated for the one and two-way scenarios with one cooperating node $N_{3}$, i.e., $M=3$. In the two scenarios and for the given set of parameters shown in Figure 3.8, the step size of adjusting the number of blocks of the two conditions is set to 900 for an interval length $T=5000$ blocks. When the divergence gap becomes less than 0.1 , the step size is changed to 200 , where the gap stays in the range between 0.005 to about 0.05 . At this small range, the performance of $N_{1}$ and 


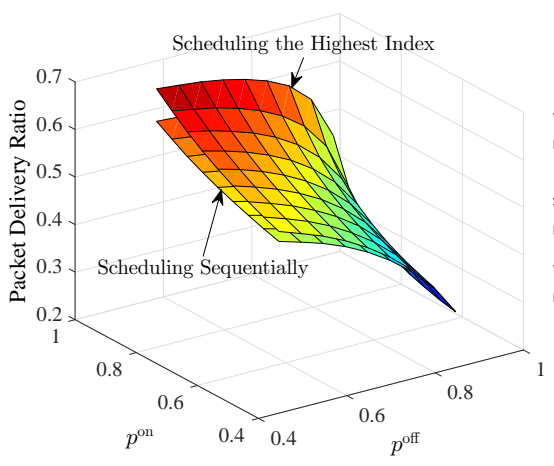

(a) Overall packet delivery ratio

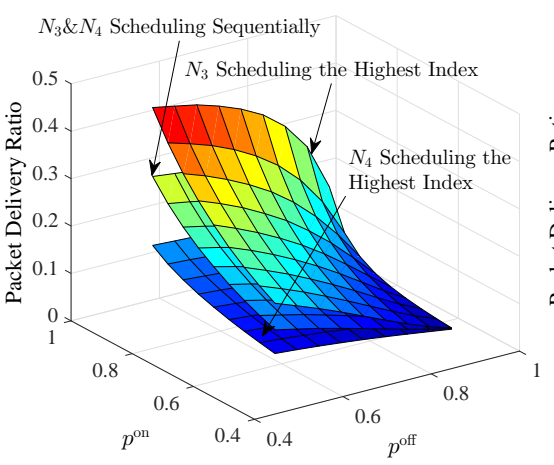

(b) $N_{3}$ and $N_{4}$ packet delivery ratios

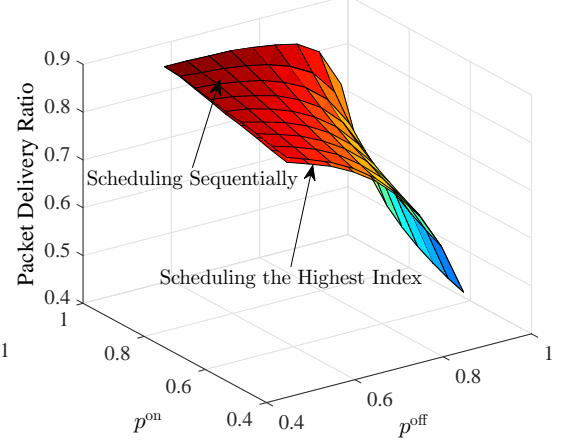

(c) $N_{1}$ packet delivery ratio

Figure 3.7: The packet delivery ratios of the two ways of cooperating nodes selection (selecting the node of the highest index and selecting the nodes sequentially) for the one-way scenario when $M=4$. Parameters used: $q_{1}^{\text {on }}=0.9, q_{1}^{\text {off }}=0.7, p_{1}^{\text {on }}=q_{3}^{\text {on }}=0.85, p_{1}^{\text {off }}=q_{3}^{\text {off }}=0.7$, $E_{1}=E_{3}=1, \delta_{1}=\delta_{3}=3, \tilde{\delta}_{1}=\tilde{\delta}_{3}=1, K=20$.

the performance $N_{3}$ are considered to be converged.

Figure 3.8 (a) illustrates the convergence between $N_{1}$ and $N_{3}$ performances of the oneway scenario. It can be noticed that the performance of the two nodes converges in the second interval. At interval 41, the performance diverges again due to the change in one of the system parameters, $\tilde{\delta}_{1}$ is changed to 3 . After two intervals, i.e., at interval 43 , the performance gets converged again by repeating the same procedures. Before the parameter change, the performance converges one block faster than after the parameter change. This is because the divergence gap between $N_{1}$ and $N_{3}$ performances in the first interval before the parameter change is smaller than it after the parameter change.

As for the two-way scenario, Figure 3.8 (b) shows the convergence in the performance between the three nodes $N_{1}, N_{2}$, and $N_{3}$. The figure shows that the performance converges twice, before and after the change of $\tilde{\delta}_{1}$. Because of the same reason in the one-way system, the convergence before the parameter change is faster than after the parameter change.

\subsection{Conclusions}

We upgrade the feedback scheme proposed in Chapter 2 for the scenarios of multiple cooperating nodes, and for one-way and two-way cooperative communications. The upgraded 


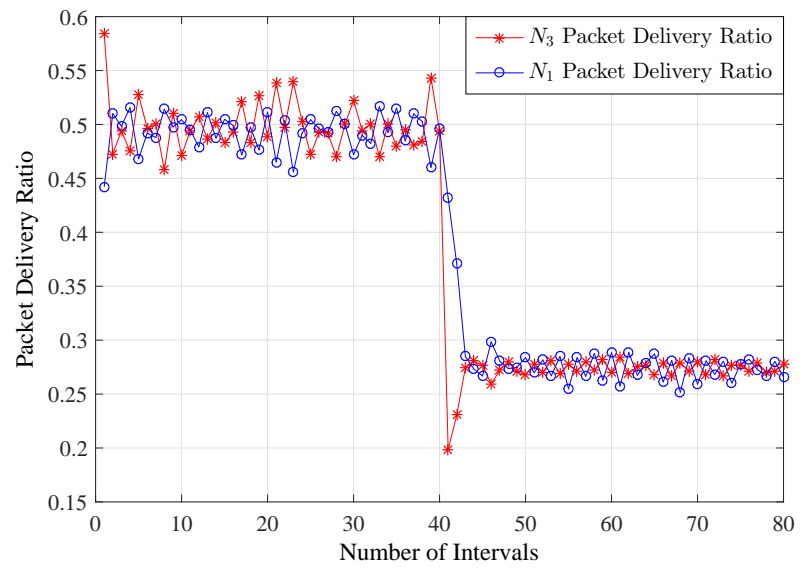

(a) One-way cooperative scenario

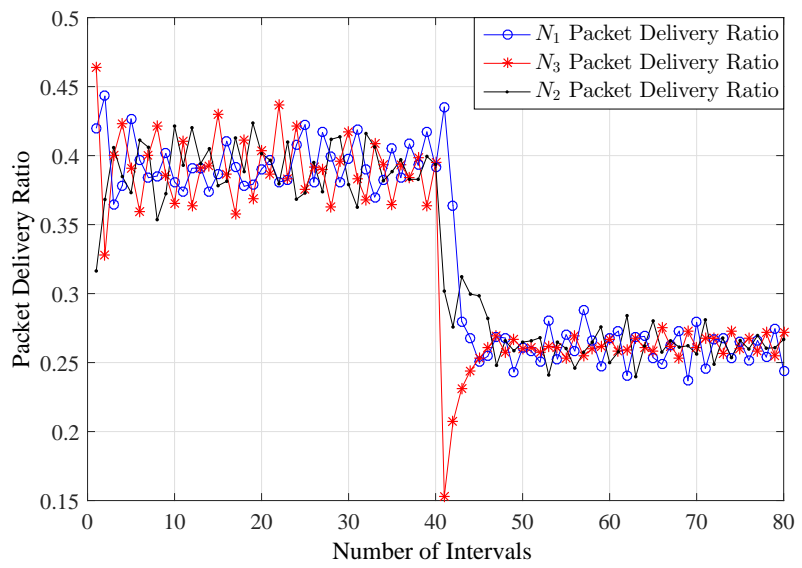

(b) Two-way cooperative scenario

Figure 3.8: The convergence of the packet delivery ratios for the one-way and two-way scenarios when $M=3$. Parameters used: $q_{1}^{\text {on }}=q_{3}^{\text {on }}=1, q_{1}^{\text {off }}=q_{3}^{\text {off }}=0, p_{1}^{\text {on }}=0.85$, $p_{3}^{\text {on }}=0.95, p_{3}^{\text {off }}=0.55, p_{1}^{\text {off }}=0.7, E_{1}=E_{3}=1, \delta_{1}=3, \tilde{\delta}_{1}=\tilde{\delta}_{3}=\delta_{3}=1, K=20$.

algorithm communicates in one or two hops, maximizing the packet delivery ratio of the system. It can be extended to three or more hops where in each hop the transmitting node selects a cooperating node from the next hop using the same algorithm. However, this requires some changes in the system model. For example, the number of time slots per a time block would need to be adjusted. In this chapter, we also propose an adaptive scheme that ensures fairness between the nodes and adapts as the parameters change. In addition, we propose a method to decrease the overhead caused by energy statuses broadcasting. Although this method reduces the overhead, it is still relatively high and might be untolerated in some applications. As a solution, in Chapter 5 we develop a statistical model that enables the source in the network to estimate the cooperating node energy status. Lastly, in the adaptive scheme, fairness is considered ensured when the nodes achieve the same performance. This might be unfair in some scenarios, for example, when the penalty on the cooperating node's performance has to be high in order to achieve the same performance as the other node. Moreover, since each transmitting node in the system is fixed and categorized a priori as either a source or a cooperating node, the adaptive scheme will not achieve fairness if the source node achieves higher performance than the cooperating node. Therefore, in the next chapter, we generalize the system model and we propose an analytical-based scheme 
that ensures fairness depending on a given penalty function while maximizing the overall performance of the system. 


\section{Chapter 4}

\section{Generalized Fairness and Optimal Scheduling}

\subsection{Introduction}

In Chapter 2, we proposed a scheduling scheme, called feedback scheme, for a network of a single cooperating node. We then upgraded this scheme in Chapter 3 to include the scenario of multiple cooperating nodes as well as the scenarios of one-way and two-way cooperative communications. In Chapter 3, we also proposed an adaptive scheduling scheme that incorporates fairness in the performance between the transmitting nodes. However, the adaptive scheme considers only the case of absolute fairness, which might be unfair in the scenarios when the penalty on the cooperating node performance has to be high in order to achieve the same performance of the source. Also, because each transmitting node in the system is fixed and categorized a priori as either a source or a cooperating node, the adaptive scheme cannot achieve fairness if the source node achieves higher performance than the cooperating node. Therefore, in this chapter, we first generalize the system model of the three-node network, where each transmitting node can be either a source, a cooperating, or a relay node depending on the system parameters. And then we propose an analyticalbased approach scheme, called fair scheduling (FS), that 1) ensures fairness between the performance of the transmitting nodes while maximizing the sum of their packet delivery ratios, and 2) considers not only the case of absolute fairness, but also the case when fairness 


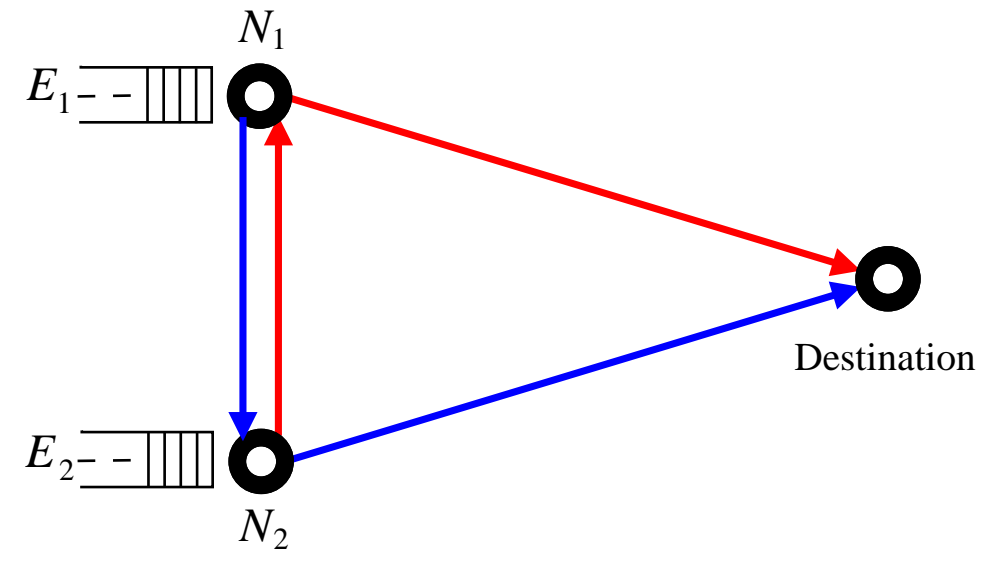

Figure 4.1: A cooperative energy harvesting sensor network of two transmitting nodes $\left(N_{1}\right.$ and $N_{2}$ ) and a destination. $E_{1}$ and $E_{2}$ are queues representing the energy harvested by $N_{1}$ and $N_{2}$, respectively, in the $n$-th time block, $n \in\{1,2, \ldots, N\}$.

is ensured when the transmitting nodes achieve performance with some difference that is determined by a given penalty function. We also propose a constraint scheduling (CS) scheme that constrains one of the transmitting nodes to achieve a certain performance.

To build the FS and CS schemes, we optimally design the system to operate in a mixed mode which is a combination of two of the three operating modes: noncooperation, cooperation, and relay. For a given penalty function, the simulation results show that the FS scheme provides the optimal operating mode for the system such that fairness is ensured for the transmitting nodes while maximizing the sum of their performances. In addition, the results show that the CS scheme provides the optimal operating mode for the system in order for a transmitting node to achieve a certain performance, if possible.

\subsection{System Model}

We consider a network of three nodes: two transmitting nodes $\left(N_{1}\right.$ and $\left.N_{2}\right)$ and a destination, as depicted in Figure 4.1. Depending on the scheduling purpose, the system operates in a mixed mode which is optimally combined of two of the three operating modes: noncooperation, cooperation, and relay, that are illustrated in Figure 4.2. In noncooperation mode, each transmitting node operates as a Source type $1\left(S_{1}\right)$ node, which transmits on its own 


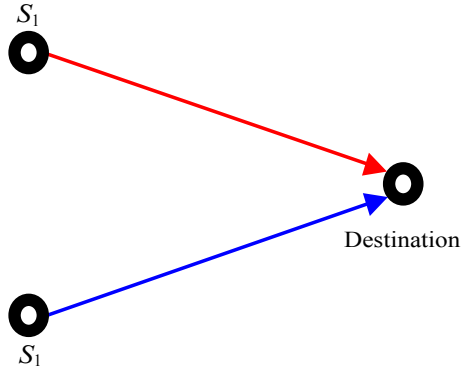

(a) Noncooperation mode

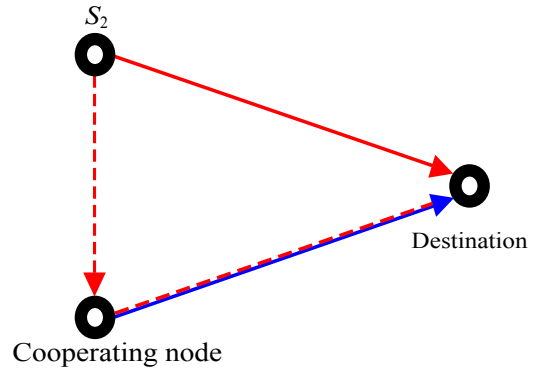

(b) Cooperation mode

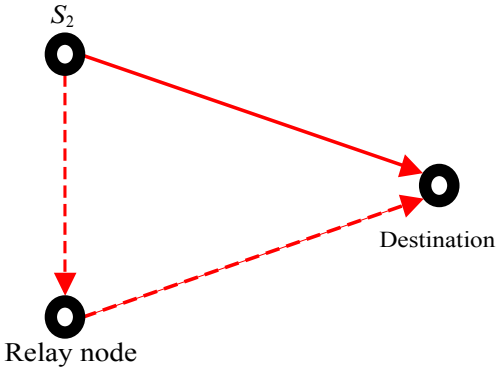

(c) Relay mode

Figure 4.2: The figure illustrates the noncooperation, cooperation, and relay modes, which are used in appropriate combinations to build the FS and CS scheduling schemes. The solid line between $S_{1}, S_{2}$, or cooperating node and destination represents the direct transmission of the event of that node to the destination. The dashed path in the cooperation and relay modes represents the relay transmission of $S_{2}$ to the destination using cooperating and relay nodes, respectively. In the relay mode, there is no solid line between the relay node and the destination because the relay node has no own transmissions where it drops all its transmissions to help with $S_{2}$ transmissions.

directly to the destination. In cooperation mode, one of the transmitting nodes operates as a Source type $2\left(S_{2}\right)$ node while the other operates as a cooperating node. The $S_{2}$ node transmits its events to destination either directly or through the cooperating node, and the cooperating node assists cooperative communication between $S_{2}$ and destination, as well as it transmits its own events directly to the destination. In relay mode, one of the transmitting nodes operates as an $S_{2}$ node similar to the cooperation mode. In the relay mode, however, the $S_{2}$ node transmits cooperatively using the other transmitting node that operates as a relay node, which discards all of its own events to help with the transmissions of the $S_{2}$ node to the destination.

The main assumptions and notations that are used for the system model throughout this chapter are:

1. Each transmitting node is equipped with energy harvesting devices and a rechargeable battery with capacity $K$.

2. We use a discrete-time model where time is divided into $N$ blocks. 
3. There is at most one event generated per transmitting node per block. We assume that sensors are monitoring different events so that their event generation processes are independent. As in [65], the event generation process of each node is modeled as a Bernoulli process, where in the time block $n, n \in\{1,2, \ldots, N\}$, node $N_{i}, i \in\{1,2\}$ generates an event $\mathcal{E}_{i}[n]=1$ with probability $q_{i}$.

4. The energy arrival process is modeled as a discrete random process. In the $n$-th block, $N_{i}, i \in\{1,2\}$, harvests $E_{i}$ amount of energy, respectively, where $E_{i} \in\{0,1, \ldots, \tilde{K}\}$ and $\tilde{K} \leqslant K$ is the maximum amount of energy that can be harvested in one block. Because a cooperation scenario is considered meaning that sensors locate close to each other and thus experience a similar environment, the energy harvesting processes of the two nodes are assumed to be correlated, where the joint probability of any two energy arrivals $E_{1}$ and $E_{2}$ is $p\left(E_{1}, E_{2}\right)$. The marginal probability of $E_{i}, i \in\{1,2\}$ is denoted by $p^{(i)}\left(E_{i}\right)$.

5. The energy harvested in the $n$-th block can be used only in a later time block.

6. We consider a real-time monitoring deployment scenario where no packets are buffered.

7. We assume that the length of each time block is enough to transmit only one event. So that in the cooperation mode, if $S_{2}$ and the cooperating node both have an event generated and sufficient energy for transmission in the same time block, the cooperating node will cooperate with $S_{2}$ and drop its transmission.

8. As in [13] and [14], the battery of a transmitting node is modeled as a buffer, and that each slot in the buffer can hold one energy unit. The maximum number of quanta that can be stored is $K$ so that the set of possible energy levels of node $N_{i}$ is $L_{i}^{x}=$ $\{0,1, \ldots, K\}, x \in\{\mathrm{n}, \mathrm{c}, \mathrm{r}\}$, where the superscript $\mathrm{n}$ is to indicate the noncooperation mode, the superscript $\mathrm{c}$ is to indicate the cooperation mode, and the superscript $\mathrm{r}$ is to indicate the relay mode.

9. We assume that $\delta_{i}$ is the energy required to reliably transmit information about the event $\mathcal{E}_{i}$ from the transmitting node $N_{i}$ to the destination, and $\tilde{\delta}$ is the energy required 
for reliable transmission between $N_{1}$ and $N_{2}$ in the cooperation and relay modes, where $\tilde{\delta}<\delta_{i}$ and $\tilde{\delta}, \delta_{i} \in\{0,1, \ldots, K\}$.

10. We denote the achieved packet delivery ratio of node $N_{i}, i \in\{1,2\}$ by $\triangle_{i} \in\left\{\triangle_{i}^{\text {n }}\right.$, $\left.\triangle_{i}^{\mathrm{c}}, \triangle_{i}^{\mathrm{r}}, \triangle_{i}^{\mathrm{m}}\right\}$, where the superscripts $\mathrm{n}, \mathrm{c}$, and $\mathrm{r}$ are as defined in Assumption 9 and the superscript $\mathrm{m}$ is to indicate the mixed mode.

\subsection{Operating Modes}

As stated earlier, the system operates in a mixed mode which is a combination of two of the three operating modes; noncooperation, cooperation, and relay. In order to design the appropriate mixed mode, depending on the scheduling purpose, we first need to study these three modes and calculate the packet delivery ratio achieved by each transmitting node in each mode.

\subsubsection{Noncooperation Mode}

In this operating mode, the transmitting node $N_{i}, i \in\{1,2\}$ operates as an $S_{1}$ node which transmits its generated event $\mathcal{E}_{i}[n], n \in\{1,2, \ldots, N\}$ on its own to the destination over the $N$ time blocks. The event $\mathcal{E}_{i}[n]$ is considered to be successfully delivered to the destination if $N_{i}$ is energized in the $n$-th time block, i.e., its energy battery level $L_{i}^{\mathrm{n}}[n] \geqslant \delta_{i}$. Thus, the packet delivery ratio $\triangle_{i}^{\mathrm{n}}$ of node $N_{i}$ over the $N$ time blocks is

$$
\triangle_{i}^{\mathrm{n}}=\frac{P\left(\mathcal{E}_{i}[n]=1\right) \operatorname{Pr}\left(L_{i}^{\mathrm{n}}[n] \geqslant \delta_{i}\right) N}{\operatorname{Pr}\left(\mathcal{E}_{i}[n]=1\right) N}=\operatorname{Pr}\left(L_{i}^{\mathrm{n}}[n] \geqslant \delta_{i}\right),
$$

and the energy battery level of the node is

$$
L_{i}^{\mathrm{n}}[n]=\min \left\{L_{i}^{\mathrm{n}}[n-1]+E_{i}[n-1]-\mathbb{1}_{\left\{L_{i}^{\mathrm{n}}[n-1] \geqslant \delta_{i}\right\}} \mathcal{E}_{i}[n-1], K\right\}
$$

where $\mathbb{1}_{\{\cdot\}}$ is the indicator function. $L_{i}^{\mathrm{n}}[n]$ is set to equal to the minimum between the two terms shown in (4.2) because of battery overflow, which might occur due to the assumption that the battery capacity of the transmitting nodes is finite. Because $L_{i}^{\mathrm{n}}[n]$ depends only on the previous energy battery level $L_{i}^{\mathrm{n}}[n-1], L_{i}^{\mathrm{n}}[n]$ is modeled as a Markov chain with 
state space $\{0,1, \ldots, K\}$, where the transition between any two states depends on $E_{i}[n-1]$ and $\mathcal{E}_{i}[n-1]$. Let $m$ and $n$ be the current and the previous states of energy battery level, respectively, and let $z=m-n$ and $w=m-n+\delta_{i}$. Then given $q_{i}$ and $p^{(i)}\left(E_{i}\right), E_{i} \in$ $\{0,1, \ldots, \tilde{K}\}$, the transition probability $P_{n, m}^{(i)}=\operatorname{Pr}\left(L_{i}[n]=m \mid L_{i}[n-1]=n\right)$ of node $N_{i}$, $i \in\{1,2\}$ between any two states $n, m \in\{0,1, \ldots, K\}$ is

$$
P_{n, m}^{(i)}= \begin{cases}p^{(i)}(z) & n<\delta_{i}, m<K \\ p^{(i)}(w) q_{i}+p^{(i)}(z)\left(1-q_{i}\right) & n \geqslant \delta_{i}, m<K \\ \sum_{k=z}^{\tilde{K}} p^{(i)}(k) I_{1} & n<\delta_{i}, m=K \\ w-1 & \\ \sum_{k=z} p^{(i)}(k)\left(1-q_{i}\right) I_{2}+\sum_{k=w}^{\tilde{K}} p^{(i)}(k) I_{3} & n \geqslant \delta_{i}, m=K,\end{cases}
$$

where $I_{1}=\mathbb{1}_{\{z \leqslant \tilde{K}\}}, I_{2}=\mathbb{1}_{\{z \leqslant w-1\}}$, and $I_{3}=\mathbb{1}_{\{w \leqslant \tilde{K}\}}$ are the indicator functions. Let $\mathbf{P}^{(i)}$ be the transition matrix of the Markov chain where its $(n, m)$-th element is given by $P_{n, m}^{(i)}$. Since this Markov chain is finite, irreducible, and aperiodic, it has average (steady state) probabilities for all states, energy battery levels. As in [65], we can obtain these average probabilities using the eigen decomposition of the matrix $\mathbf{P}^{(i)}$. Such that, the eigen vector $\boldsymbol{\pi}^{(i)}=\left[\pi_{0}^{(i)}, \pi_{1}^{(i)}, \ldots, \pi_{K}^{(i)}\right]^{\top}$ of $\mathbf{P}^{(i)}$ that corresponds to its unity eigen value represents the average state probabilities, where $\pi_{i, k}, k=\{0, \ldots, K\}$ is the average probability of the $k$ th energy battery level of node $N_{i}, i \in\{1,2\}$. Using the average probabilities, the packet delivery ratio of $N_{i}$ in (4.1) becomes

$$
\triangle_{i}^{\mathrm{n}}=\operatorname{Pr}\left(L_{i}^{\mathrm{n}} \geqslant \delta_{i}\right)=\sum_{k=\delta_{i}}^{K} \pi_{k}^{(i)} .
$$

\subsubsection{Cooperation Mode}

In cooperation mode, one of the transmitting nodes, say $N_{i}, i \in\{1,2\}$, operates as an $S_{2}$ while the other transmitting node $N_{j}, j \in\{1,2\}, i \neq j$, operates as a cooperating node. As stated in Section 4.2, the $S_{2}$ node transmits its events to destination either directly or through the cooperating node, and the cooperating node assists cooperative communication between $S_{2}$ and destination, as well as it transmits its own events to the destination. Algorithm 4.1 explains the cooperation scenario of $N_{j}$ with $N_{i}$. It is similar to Algorithm 2.1, however, 


\begin{tabular}{|c|c|}
\hline \multicolumn{2}{|l|}{ Algorithm 4.1 Cooperation Mode } \\
\hline \multicolumn{2}{|l|}{ (a) $S_{2}$ node $N_{i}, i \in\{1,2\}$} \\
\hline 1: Inputs: $\mathcal{E}_{i}[n], L_{i}^{\mathrm{c}}[n], A_{j}^{\mathrm{c}}[n], \tilde{\delta}, \delta_{i}$ & 1: Inputs: $\mathcal{E}_{j}[n], L_{j}^{\mathrm{c}}[n], \delta_{j}$ \\
\hline 2: Outputs: Transmission action of $N_{i}$; & 2: Outputs: Transmission action of $N_{j}$; \\
\hline $3: \quad$ if $\mathcal{E}_{i}[n]=1$ then & $3:$ if $L_{j}^{\mathrm{c}}[n] \geqslant \delta_{j}$ then \\
\hline if $L_{i}^{\mathrm{c}}[n] \geqslant \delta_{i}$ AND $A_{j}^{\mathrm{c}}[n]=0$ then & 4: $\quad$ Sense the link $N_{i}-N_{j}$ for $N_{i}$ action; \\
\hline$N_{i}$ action $=$ direct transmission & $5: \quad$ if $N_{i}$ action $=$ relay transmission then \\
\hline elseif $L_{i}^{\mathrm{c}}[n] \geqslant \tilde{\delta} \operatorname{AND} A_{j}^{\mathrm{c}}[n]=1$ then & $N_{j}$ action $=$ relay transmission \\
\hline$N_{i}$ action $=$ relay transmission & $N_{j}^{\prime}$ 's event will be discarded, if any; \\
\hline else & elseif $N_{i}$ action $=$ no transmission then \\
\hline$N_{i}$ action $=$ no transmission & if $\mathcal{E}_{j}[n]=1$ then \\
\hline 10: endif; & $N_{j}$ action $=$ own-traffic transmission \\
\hline 11: else & else \\
\hline$N_{i}$ action $=$ no transmission & $N_{j}$ action $=$ no transmission \\
\hline 13: endif & endif \\
\hline & 14: endif \\
\hline & 15: else \\
\hline & 16: $\quad N_{j}$ action $=$ no transmission \\
\hline & 17: endif \\
\hline
\end{tabular}


here there is no need for using a TDMA protocol because of the asumption that only one event is reported per time block. As can be seen in Algorithm 4.1, the decision on the $S_{2}$ transmission action in the $n$-th time block is made based on the energy status $A_{j}^{\mathrm{c}}$ of the cooperating node $N_{j}$, which is given by

$$
A_{j}^{\mathrm{c}}[n]= \begin{cases}1 & \text { if } L_{j}^{\mathrm{c}}[n] \geqslant \delta_{j} \\ 0 & \text { otherwise }\end{cases}
$$

Using $A_{j}^{\mathrm{c}}[n]$ as shown in part (a) of the algorithm, the $S_{2}$ node $N_{i}$ in each time block takes one of three transmission actions: direct transmission, relay transmission, or no transmission. In direct transmission action, $S_{2}$ transmits its event directly to the destination if it has an event generated $\mathcal{E}_{i}[n]=1$, it is energized $L_{i}^{\mathrm{c}}[n] \geqslant \delta_{i}$, and the cooperating node $N_{j}$ is not energized $A_{j}^{\mathrm{c}}[n]=0$. In relay transmission action, $S_{2}$ transmits its event to the destination using the cooperating node if it has an event generated $\mathcal{E}_{i}[n]=1$, it is energized for relay transmission $L_{i}^{\mathrm{c}}[n] \geqslant \tilde{\delta}$, and the cooperating node $N_{j}$ is energized $A_{j}^{\mathrm{c}}[n]=1$. In both of these two transmission actions, the event of $S_{2}$ is reported successfully to the destination. If none of these two transmission actions is taken, the transmission action of $S_{2}$ will be no transmission where no event will be reported for $S_{2}$.

As shown in part (b) of the algorithm, the cooperating node $N_{j}$ also takes one of three transmission actions: own-traf fic transmission, relay transmission, or no transmission. In each time block, if $L_{j}^{\mathrm{c}}[n] \geqslant \delta_{j}$, the cooperating node senses the transmission link $N_{i}-N_{j}$. If there is a transmission, i.e., $S_{2}$ has a relay transmission, the cooperating node takes relay transmission action to forward the transmission of $S_{2}$. In this scenario, if the cooperating node has an event generated, its event will be discarded due to the assumption that only one event is reported per time block. If there is no transmission, the cooperating node takes own - traffic transmission action if it has an event generated $\mathcal{E}_{j}[n]=1$, or no transmission action if it has no event generated $\mathcal{E}_{j}[n]=0$. If the own-traffic transmission action is taken, the event of the cooperating node is reported successfully to the basestation. If $L_{j}^{\mathrm{c}}[n]<\delta_{j}$, the cooperating node does not need to sense the transmission link $N_{i}-N_{j}$ and takes no transmission action, where no event will be reported for the cooperating node.

Let $d_{1}^{\mathrm{c}}$ and $d_{2}^{\mathrm{c}}$ be the events of the direct and relay transmission actions of the $S_{2}$ node 
$N_{i}$, respectively, and let $d_{3}^{\mathrm{c}}$ and $d_{4}^{\mathrm{c}}$ be the events of the own-traffic and relay transmission actions of the cooperating node $N_{j}$, where from Algorithm 4.1

$$
\begin{gathered}
d_{1}^{\mathrm{c}}[n]= \begin{cases}1 & \text { if }\left(\mathcal{E}_{i}[n]=1 \cap L_{i}^{\mathrm{c}}[n] \geqslant \delta_{i} \cap A_{j}^{\mathrm{c}}[n]=0\right) \\
0 & \text { otherwise, }\end{cases} \\
d_{2}^{\mathrm{c}}[n]= \begin{cases}1 & \text { if }\left(\mathcal{E}_{i}[n]=1 \cap L_{i}^{\mathrm{c}}[n] \geqslant \tilde{\delta} \cap A_{j}^{\mathrm{c}}[n]=1\right) \\
0 & \text { otherwise, }\end{cases} \\
d_{3}^{\mathrm{c}}[n]= \begin{cases}1 & \text { if }\left(\mathcal{E}_{j}[n]=1 \cap L_{j}^{\mathrm{c}}[n] \geqslant \delta_{j}\right) \cap\left(\mathcal{E}_{i}[n]=0 \cup L_{i}^{\mathrm{c}}[n]<\tilde{\delta}\right) \\
0 & \text { otherwise, }\end{cases}
\end{gathered}
$$

and $d_{4}^{\mathrm{c}}=d_{2}^{\mathrm{c}}$. Then, the energy battery level $L_{i}^{\mathrm{c}}$ of $N_{i}$ is

$$
L_{i}^{\mathrm{c}}[n]=\min \left\{L_{i}^{\mathrm{c}}[n-1]+E_{i}[n-1]-\tilde{\delta} d_{2}^{\mathrm{c}}[n-1]-\delta_{i} d_{1}^{\mathrm{c}}[n-1], K\right\},
$$

and the energy battery level $L_{j}^{\mathrm{c}}$ of $N_{j}$ is

$$
L_{j}^{\mathrm{c}}[n]=\min \left\{L_{j}^{\mathrm{c}}[n-1]+E_{j}[n-1]-\delta_{j} d_{4}^{\mathrm{c}}[n-1]-\delta_{j} d_{3}^{\mathrm{c}}[n-1], K\right\}
$$

The current energy battery levels $L_{i}^{\mathrm{c}}[n]$ and $L_{j}^{\mathrm{c}}[n]$ in (4.9) and (4.10), respectively, are dependent, and each depends only on the energy battery levels of the previous time block $L_{i}^{\mathrm{c}}[n-1]$ and $L_{j}^{\mathrm{c}}[n-1]$, so that their joint distribution is modeled as a Markov chain with a state space $\{(0,0),(0,1), \ldots,(0, K),(1,0), \ldots,(1, K), \ldots,(K, 0), \ldots,(K, K)\}$. Let $m$ and $n$, $m, n \in\{0,1, \ldots, K\}$ be the previous energy battery levels of the source and cooperating nodes $L_{i}^{\mathrm{c}}[n-1]$ and $L_{j}^{\mathrm{c}}[n-1]$, respectively, and let $u$ and $v, u, v \in\{0,1, \ldots, K\}$ be their current energy battery levels $L_{i}^{\mathrm{c}}[n]$ and $L_{j}^{\mathrm{c}}[n]$, respectively. Define $w=u-m$ and $z=v-n$. Then from (4.9) and (4.10), the transition probability $P_{s_{1}, s_{2}}=\operatorname{Pr}\left(s_{1}=(u, v) \mid s_{2}=(m, n)\right)$ from state $(m, n)$ to state $(u, v)$ if $u<K$ and $v<K$ is

$$
P_{s_{1}, s_{2}}= \begin{cases}p(w, z) & m<\delta_{i}, n<\delta_{j}, \\ p\left(w, z+\delta_{j}\right) q^{r}+p(w, z)\left(1-q^{r}\right) & m<\tilde{\delta}, n \geqslant \delta_{j}, \\ p\left(w+\tilde{\delta}, z+\delta_{j}\right) q^{s}+\left(p\left(w, z+\delta_{j}\right) q^{r}+p(w, z)\left(1-q^{r}\right)\right)\left(1-q^{s}\right) & m \geqslant \tilde{\delta}, n \geqslant \delta_{j}, \\ p\left(w+\delta_{i}, z\right) q^{s}+p(w, z)\left(1-q^{s}\right) & m \geqslant \delta_{i}, n<\delta_{j},\end{cases}
$$


where the first case is for the scenario when none of the nodes has sufficient energy for any transmission; the second case is for the scenario when the source node has no sufficient energy for any transmission while the cooperating node does; the third case is for the scenario when the source node and cooperating node both have has sufficient energy for cooperative transmission, and the fourth case is for the scenario when the source has sufficient energy for direct transmission and the cooperating node has no sufficient energy for any transmission. In each of these four cases, if $u=K$ and/or $v=K$, the transition from state $(m, n)$ to state $(u, v)$ will include all events that energy arrival in the previous time block can cause battery overflow. For example, in the first case when $m<\delta_{i}$ and $n<\delta_{j}, P_{s_{1}, s_{2}}=\sum_{x=z}^{\tilde{K}} p_{(w, x)}$ if $u<K$ and $v=K, P_{s_{1}, s_{2}}=\sum_{y=w}^{\tilde{K}} p_{(y, z)}$ if $u=K$ and $v<K$, and $P_{s_{1}, s_{2}}=\sum_{y=w}^{\tilde{K}} \sum_{x=z}^{\tilde{K}} p_{(y, x)}$ if $u=K$ and $v=K$.

Let $\mathbf{P}$ be the transition matrix of the Markov chain where its elements are given by $P_{s_{1}, s_{2}}$. Then, we can obtain the marginal probabilities $\operatorname{Pr}\left(L_{i}^{\mathrm{c}}\right)$ and $\operatorname{Pr}\left(L_{j}^{\mathrm{c}}\right)$ as well as the joint probability $\operatorname{Pr}\left(L_{i}^{\mathrm{c}}, L_{j}^{\mathrm{c}}\right)$ using eigen decomposition of $\mathbf{P}$. Using these probabilities, the packet delivery ratio $\triangle_{i}^{\mathrm{c}}$ of $S_{2}$ is

$$
\begin{gathered}
\triangle_{i}^{\mathrm{c}}=\frac{\left[\operatorname{Pr}\left(d_{1}^{\mathrm{c}}=1\right)+\operatorname{Pr}\left(d_{2}^{\mathrm{c}}=1\right)\right] N}{\operatorname{Pr}\left(\mathcal{E}_{i}=1\right) N}, \\
\triangle_{i}^{\mathrm{c}}=\operatorname{Pr}\left(L_{i}^{\mathrm{c}} \geqslant \delta_{i} \cap L_{j}^{\mathrm{c}}<\delta_{j}\right)+\operatorname{Pr}\left(L_{i}^{\mathrm{c}} \geqslant \tilde{\delta} \cap L_{j}^{\mathrm{c}} \geqslant \delta_{j}\right),
\end{gathered}
$$

and the packet delivery ratio $\triangle_{j}^{\mathrm{c}}$ of the cooperating node is

$$
\begin{gathered}
\triangle_{j}^{\mathrm{c}}=\frac{\operatorname{Pr}\left(d_{3}^{\mathrm{c}}=1\right) N}{\operatorname{Pr}\left(\mathcal{E}_{i}=1\right) N}, \\
\triangle_{j}^{\mathrm{c}}=\left(1-q_{i}\right) \operatorname{Pr}\left(L_{j}^{\mathrm{c}} \geqslant \delta_{j}\right)+\operatorname{Pr}\left(L_{i}^{\mathrm{c}}<\tilde{\delta} \cap L_{j}^{\mathrm{c}} \geqslant \delta_{j}\right)- \\
\left(1-q_{i}\right) \operatorname{Pr}\left(L_{i}^{\mathrm{c}}<\tilde{\delta} \cap L_{j}^{\mathrm{c}} \geqslant \delta_{j}\right) .
\end{gathered}
$$

\subsubsection{Relay mode}

The relay mode is similar to the cooperation mode. However, instead of operating as a cooperating node, node $N_{j}$ in the relay mode operates as a relay node, which drops all its own transmissions to help with the transmissions of node $N_{i}$, the $S_{2}$ node. Algorithm 
4.1 can represent the relay mode if the lines (9-13) in part (b) are replaced by the line: $N_{j}$ action $=$ no transmission, and superscript $\mathrm{c}$ is replaced by $\mathrm{r}$ in the two parts. Consequently, the $S_{2}$ node $N_{i}$ will still have the same three transmission actions, while the relay node $N_{j}$ will have two transmission actions instead of the three that are relay transmission and no transmission.

Let $A_{j}^{\mathrm{r}}$ be the energy status of the relay node, then the events of the direct and relay transmission actions $d_{1}^{\mathrm{r}}$ and $d_{2}^{\mathrm{r}}$, respectively, of the $S_{2}$ node $N_{i}$ in the $n$-th time block are

$$
\begin{aligned}
& d_{1}^{\mathrm{r}}[n]= \begin{cases}1 & \text { if }\left(\mathcal{E}_{i}[n]=1 \cap L_{i}^{\mathrm{r}}[n] \geqslant \delta_{i} \cap A_{j}^{\mathrm{r}}[n]=0\right) \\
0 & \text { otherwise, }\end{cases} \\
& d_{2}^{\mathrm{r}}[n]= \begin{cases}1 & \text { if }\left(\mathcal{E}_{i}[n]=1 \cap L_{i}^{\mathrm{r}}[n] \geqslant \tilde{\delta} \cap A_{j}^{\mathrm{r}}[n]=1\right) \\
0 & \text { otherwise, }\end{cases}
\end{aligned}
$$

and the event of the relay transmission action of the relay node $N_{j} d_{4}^{\mathrm{r}}[n]=d_{2}^{\mathrm{r}}[n]$. As a result, the energy battery level $L_{i}^{\mathrm{r}}$ of $N_{i}$ is

$$
L_{i}^{\mathrm{r}}[n]=\min \left\{L_{i}^{\mathrm{r}}[n-1]+E_{i}[n-1]-\tilde{\delta} d_{2}^{\mathrm{r}}[n-1]-\delta_{i} d_{1}^{\mathrm{r}}[n-1], K\right\},
$$

and the energy battery level $L_{j}^{\mathrm{r}}$ of $N_{j}$ is

$$
L_{j}^{\mathrm{r}}[n]=\min \left\{L_{j}^{\mathrm{r}}[n-1]+E_{j}[n-1]-\delta_{j} d_{4}^{\mathrm{r}}[n-1], K\right\},
$$

As in cooperation mode, the joint distribution of $L_{i}^{\mathrm{r}}[n]$ and $L_{j}^{\mathrm{r}}[n]$ is modeled as a Markov chain with the same state space. The transition matrix of $L_{i}^{\mathrm{c}}$ and $L_{j}^{\mathrm{c}}$ described in (4.11) can represent the transition matrix of $L_{i}^{\mathrm{r}}$ and $L_{j}^{\mathrm{r}}$ if we set $q^{r}=1$ and replace $z+\delta_{j}$ by $z$ only in the terms that have $q^{r}$. From the transition matrix, we can find the probabilities $\operatorname{Pr}\left(L_{i}^{\mathrm{r}}\right)$, $\operatorname{Pr}\left(L_{j}^{\mathrm{r}}\right)$, and $\operatorname{Pr}\left(L_{i}^{\mathrm{r}}, L_{j}^{\mathrm{r}}\right)$. Using these probabilities, the packet delivery ratio $\triangle_{i}^{\mathrm{r}}$ of $S_{2}$ is

$$
\begin{gathered}
\triangle_{i}^{\mathrm{r}}=\frac{\left[\operatorname{Pr}\left(d_{1}^{\mathrm{r}}=1\right)+\operatorname{Pr}\left(d_{2}^{\mathrm{r}}=1\right)\right] N}{\operatorname{Pr}\left(\mathcal{E}_{i}=1\right) N}, \\
\triangle_{i}^{\mathrm{r}}=\operatorname{Pr}\left(L_{i}^{\mathrm{r}} \geqslant \delta_{i} \cap L_{j}^{\mathrm{r}}<\delta_{j}\right)+\operatorname{Pr}\left(L_{i}^{\mathrm{r}} \geqslant \tilde{\delta} \cap L_{j}^{\mathrm{r}} \geqslant \delta_{j}\right),
\end{gathered}
$$

and the packet delivery ratio of the relay node $\triangle_{j}^{\mathrm{r}}=0$, which is due to the fact that the relay node drops all its own transmissions to help with the transmissions of $S_{2}$. 
To compare the packet delivery ratios achieved by each transmitting node in the three previous modes, assume that the transmitting node $N_{i}, i \in\{1,2\}$ and the transmitting node $N_{j}, j \in\{1,2\}, j \neq i$, each operates as an $S_{1}$ node in the noncooperation mode, and $N_{i}$ operates as an $S_{2}$ node in cooperation and relay modes and $N_{j}$ operates as a cooperating node and as a relay node in cooperation and relay modes, respectively. Then for any given set of parameters, the performance of node $N_{i}$ in relay mode is higher than or equal to its performance in cooperation mode, and this is higher than or equal to its performance in noncooperation mode, i.e., $\triangle_{i}^{\mathrm{r}} \geqslant \triangle_{i}^{\mathrm{c}} \geqslant \triangle_{i}^{\mathrm{n}}$. This holds the opposite in the case of node $N_{j}$ where its performance in noncooperation mode is higher than or equal to its performance in cooperation mode, and this is higher than or equal to its performance in relay mode, i.e., $\triangle_{j}^{\mathrm{n}} \geqslant \triangle_{j}^{\mathrm{c}} \geqslant \triangle_{j}^{\mathrm{r}}=0$. This is because in cooperation mode $S_{2}$ sometimes transmits with lower energy via node $N_{j}$, which saves $S_{2}$ some amount of energy that can be used to transmit more events than in noncooperation mode. In relay mode, $S_{2}$ transmits more frequently via node $N_{j}$ than in cooperation mode. Thus, more energy will be saved and more events will be transmitted. As for node $N_{j}$, it achieves lower performance in cooperation mode than in noncooperation mode for two reasons. First, some of its harvested energy will rather be used to forward some events of $S_{2}$ than transmitting its own. Second, some events of $N_{j}$ will be dropped even when enough energy is available due to Assumption 8. In relay mode, node $N_{j}$ spends all its harvested energy to forward the events of $S_{2}$ by dropping all its generated events. Therefore, its performance in relay mode is lower than cooperation and noncooperation modes.

\subsubsection{Mixed mode}

In mixed mode, the system operates in two of the three previous modes over the $N$ time blocks. Such that, the system operates in the first mode in the first $n$ time blocks. After that, i.e., in the remaining $N-n$ blocks, the system operates in the second mode, so that the packet delivery ratio of each node will be the summation of its successfully delivered events in the two modes over its total generated events in the $N$ time blocks. For any mixed mode, let $x, y \in\{\mathrm{n}, \mathrm{c}, \mathrm{r}\}, x \neq y$, be the first and the second operating modes, respectively, 
where $\mathrm{n}, \mathrm{c}$, and $\mathrm{r}$ are as defined in Assumption 9. Then, the packet delivery ratio of the transmitting node $N_{i}, i \in\{1,2\}$ of that mixed mode will be

$$
\triangle_{i}^{\mathrm{m}}(x, y, n)=\frac{\triangle_{i}^{x} n+(N-n) \triangle_{i}^{y}}{N} .
$$

From (4.22) if $n=N$, the system will operate in $x$ mode regardless of $y$, and the node will achieve the packet delivery ratio $\triangle_{i}^{x}$. In contrast, if $n=0$, the system will operate in $y$ mode regardless of $x$, and the node will achieve the packet delivery ratio $\triangle_{i}^{y}$. In the first case $y$ is considered do not care while in the second case $x$ is considered do not care.

\subsection{Scheduling Schemes}

\subsubsection{Fair Scheduling (FS) Scheme}

In noncooperation mode, one of the transmitting nodes may achieve lower packet delivery ratio than the other. Thus, it will successfully deliver lower percentage of its total generated events to the destination over the $N$ time blocks. Therefore, here we address the issue of event delivering with fairness from the transmitting nodes to the destination while providing the optimal sum of their packet delivery ratios. The optimal or fair packet delivery ratios can be achieved by providing the optimal scheduling or the optimal operating mode for the system.

Assume that in noncooperation mode, node $N_{j}, j \in\{1,2\}$ achieves a packet delivery ratio that is higher than or equal to node $N_{i}, i \in\{1,2\}, i \neq j$. Then for fairness, intuitively node $N_{j}$ should cooperate with or relay for node $N_{i}$, but by how much? Let the system operate in mixed $x$-n mode, where $x \in\{\mathrm{c}, \mathrm{r}\}$, and let node $N_{i}$ operate as an $S_{2}$ in $x$ mode as it achieves lower performance in noncooperation mode. Using (4.22), the packet delivery ratios $\triangle_{i}$ and $\triangle_{j}$ of $N_{i}$ and $N_{j}$, respectively, achieved in the mixed $x$-n mode over the $N$ time blocks can be expressed as

$$
\begin{aligned}
& \triangle_{i}=\triangle_{i}^{\mathrm{n}}+\frac{n\left(\triangle_{i}^{x}-\triangle_{i}^{\mathrm{n}}\right)}{N}, \\
& \triangle_{j}=\triangle_{j}^{\mathrm{n}}+\frac{n\left(\triangle_{j}^{x}-\triangle_{j}^{\mathrm{n}}\right)}{N} .
\end{aligned}
$$


Because in $x$ mode node $N_{i}$ operates as an $S_{2}$ and node $N_{j}$ operates as a cooperating or relay node depending on $x$, we have that $\triangle_{i}^{\mathrm{r}} \geqslant \triangle_{i}^{\mathrm{c}} \geqslant \triangle_{i}^{\mathrm{n}}$ and $\triangle_{j}^{\mathrm{n}} \geqslant \triangle_{j}^{\mathrm{c}} \geqslant \triangle_{j}^{\mathrm{r}}$ as explained in Section 4.3.3. Thus, from (4.23) and (4.24), as $n$ increases for a given $x \in\{\mathrm{c}, \mathrm{r}\}$, the packet delivery ratio of node $N_{i}$ increases linearly while the packet delivery ratio of $N_{j}$ decreases linearly. This implies that as $n$ increases, the difference between the performance of the two nodes decreases until it reaches to its minimum at the value of $n$ where $\triangle_{i}$ and $\triangle_{j}$ intersect. After that, the difference will start increasing because the performance of $N_{j}$ becomes lower than $N_{i}$. Hence, before the difference is minimized, the more node $N_{j}$ cooperates with or relays for node $N_{i}$, the better fairness is achieved in the performance of the two nodes. However, the higher penalty is in the decrease of node $N_{j}$ 's performance. Therefore, in order to compromise, we achieve fairness based on a given penalty function while maximizing the sum of the packet delivery ratios of the nodes. Define the penalty function $\Gamma\left(\left|\triangle_{j}-\triangle_{i}\right|\right)$ as a monotonic increasing function in the absolute difference between the performance of the two nodes, where as $\Gamma\left(\left|\triangle_{j}-\triangle_{i}\right|\right)$ decreases, the better fairness is achieved and the higher penalty is in the decrease of node $N_{j}$ 's performance. For a given $\Gamma\left(\left|\triangle_{j}-\triangle_{i}\right|\right)$, the problem of fairness under the constraints that $x \in\{\mathrm{c}, \mathrm{r}\}$ and $0 \leqslant n \leqslant N$ is formulated as

$$
\begin{array}{ll}
\max _{\triangle_{j}, \triangle_{i}} & \max _{n, x} \quad \triangle_{j}+\triangle_{i}-\Gamma\left(\left|\triangle_{j}-\triangle_{i}\right|\right), \\
\text { subject to } & x \in\{\mathrm{c}, \mathrm{r}\}, \\
& 0 \leqslant n \leqslant N .
\end{array}
$$

For a given $x \in\{\mathrm{c}, \mathrm{r}\}$, we have from (4.23) and (4.24) that $\left(\triangle_{j}+\triangle_{i}\right)$ is a linear function that decays as $n$ increases. Therefore, because $\Gamma\left(\left|\triangle_{j}-\triangle_{i}\right|\right)$ is a convex function that monotonically increases from both sides of its minimum, the cost function in (4.25) will be concave over $n$ for a given $x$, and its maximum is at some value of $n$ that is less than the value of $n$ at which $\triangle_{i}$ and $\triangle_{j}$ intersect. Note that because the cost function is concave in both when $x=\mathrm{c}$ and $x=\mathrm{r}$, it is also maximized over $\triangle_{i}$ and $\triangle_{j}$ as can be seen from (4.25).

Define $n^{\mathrm{c}}$ and $n^{\mathrm{r}}$ as the values of $n$ that maximizes the cost function when $x=\mathrm{c}$ and when $x=\mathrm{r}$, respectively, and these values can be solved for by taking the derivative of the cost function and setting it to 0 . For some penalty functions, $n^{\mathrm{c}}$ can be a value that is higher than $N$. This happens when node $N_{i}$ harvests energy with a rate that is much lower than its 


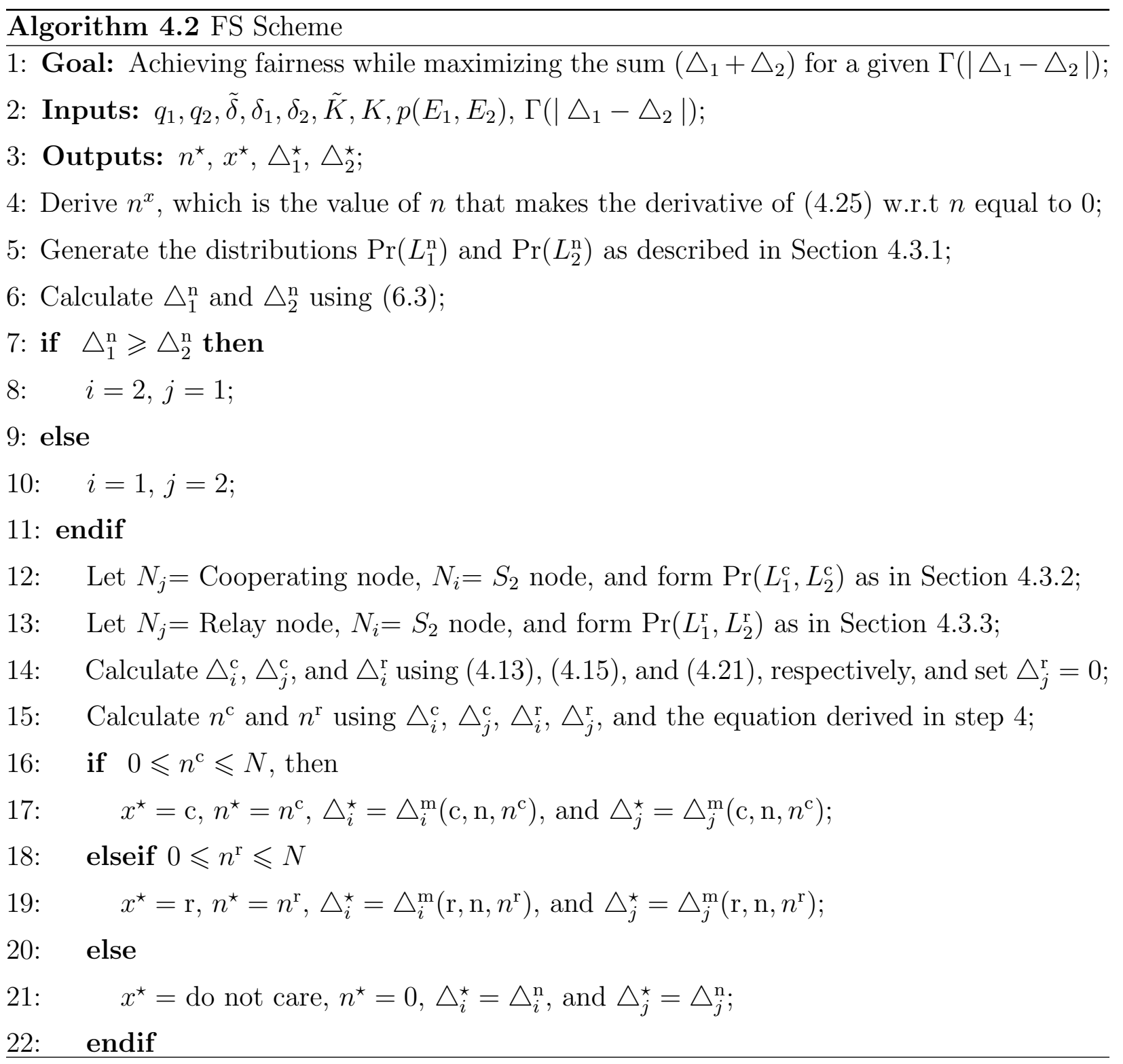


event generation rate, where $\triangle_{i}$ will still be lower than $\triangle_{j}$ even when $N_{j}$ cooperates with $N_{i}$ the all $N$ time blocks. In this case, $\triangle_{i}$ and $\triangle_{j}$ will intersect at a value of $n>N$, which results in $n^{\mathrm{c}}$ to be higher than $N$. This holds the opposite for $n^{\mathrm{r}}$, which is always a value that is less than $N$, and this is because $\triangle_{i}$ and $\triangle_{j}$ will always intersect at some value of $0 \leqslant n \leqslant N$ due to the fact that in relay mode $\triangle_{j}^{\mathrm{r}}=0$. On the other hand, for some penalty functions, $n^{x}, x \in\{\mathrm{c}, \mathrm{r}\}$ can be a value that is less than 0 , which occurs when fairness for the given penalty function needs to be achieved when the difference between $\triangle_{j}$ and $\triangle_{i}$ is higher than $\triangle_{j}^{\mathrm{n}}-\triangle_{i}^{\mathrm{n}}$. Because $\triangle_{j}^{\mathrm{n}}-\triangle_{i}^{\mathrm{n}}$, which occurs when $n=0$, is the maximum difference over the range that is less than the value of $n$ at which $\triangle_{i}$ and $\triangle_{j}$ intersect, achieving a difference that is higher than $\triangle_{j}^{\mathrm{n}}-\triangle_{i}^{\mathrm{n}}$ can happen only if $n^{x}$ is less than 0 .

The optimal solution $n^{\star}$ and $x^{\star}$ for the cost function in (4.25) is determined based on the location of its maximum when $x=\mathrm{c}$ and its maximum when $x=\mathrm{r}$, i.e., $n^{\mathrm{c}}$ and $n^{\mathrm{r}}$. If $n^{\mathrm{c}} \in[0, N]$, then $x^{\star}=\mathrm{c}$ and $n^{\star}=n^{\mathrm{c}}$ regardless of where the cost function is maximized at when $x=\mathrm{r}$. This is because when $x=\mathrm{c},\left(\triangle_{j}+\triangle_{i}\right)$ decays slower than when $x=\mathrm{r}$ due the fact that in relay mode $\triangle_{j}^{\mathrm{r}}=0$. Thus, the maximum of the cost function when $x=\mathrm{c}$ will be higher than when $x=\mathrm{r}$ for the same penalty function, so that the optimal solution is when $x^{\star}=\mathrm{c}$ and $n^{\star}=n^{\mathrm{c}}$. If $n^{\mathrm{r}} \in[0, N]$ and $n^{\mathrm{c}} \notin[0, N]$, then $x^{\star}=\mathrm{r}$ and $n^{\star}=n^{\mathrm{r}}$. If $n^{\mathrm{c}}, n^{\mathrm{r}}<0$, then $n^{\star}=0$ and $x^{\star}$ is do not care. In this case, the system will operate in noncooperation mode where the closest performance to the optimal will be achieved. The values $x^{\star}$ and $n^{\star}$ provide the optimal mixed mode of the system for the given penalty function, and by using them, the optimal performance of the two nodes $\triangle_{i}^{*}$ and $\triangle_{j}^{*}$ can be calculated using (4.23) and (4.24), respectively. Algorithm 4.2 summarizes the process of how to find the optimal solution for the cost function in (4.25).

As a simple example, we can set $\Gamma=\left|\triangle_{j}-\triangle_{i}\right|^{\alpha}$, where $\alpha>1$. By taking the derivative of the cost function in (4.25) containing this monotonic increasing penalty function, the value of $n$ that maximizes the cost function for a given $x \in\{\mathrm{c}, \mathrm{r}\}$ is

$$
n^{x}=\frac{N}{\left(\triangle_{j}^{x}-\triangle_{j}^{\mathrm{n}}\right)-\left(\triangle_{i}^{x}-\triangle_{i}^{\mathrm{n}}\right)}\left[\left(\frac{\left(\triangle_{j}^{x}-\triangle_{j}^{\mathrm{n}}\right)+\left(\triangle_{i}^{x}-\triangle_{i}^{\mathrm{n}}\right)}{\alpha\left[\left(\triangle_{j}^{x}-\triangle_{j}^{\mathrm{n}}\right)-\left(\triangle_{i}^{x}-\triangle_{i}^{\mathrm{n}}\right)\right]}\right)^{\alpha-1}-\left(\triangle_{j}^{n}-\triangle_{i}^{n}\right)\right] .
$$

By following Algorithm 4.2, the optimal value of $x$ and the optimal value of $n$ can be found for any given set of system parameters as we will see later in the simulation results. 


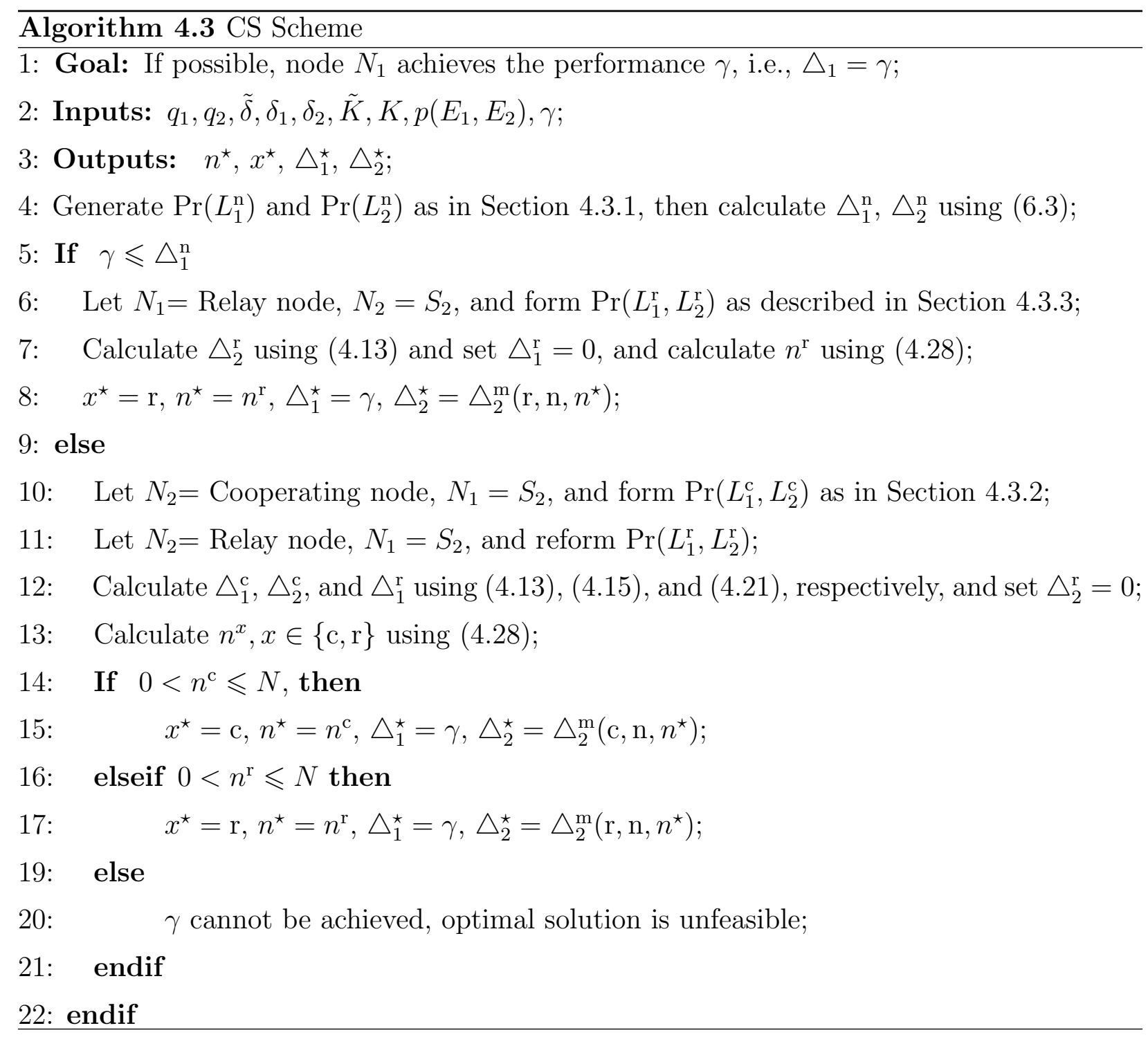




\subsubsection{Constrained Scheduling (CS) Scheme}

In some applications, one of the transmitting nodes, say node $N_{i}, i \in\{1,2\}$ may need to successfully report the average $0 \leqslant \gamma \leqslant 1$ of its total generated events to the destination over the $N$ time blocks, which can happen only if node $N_{i}$ achieves the packet delivery ratio $\triangle_{i}=\gamma$. Depending on its energy harvesting rate, node $N_{i}$ might be able to achieve the performance $\gamma$ either by its own or by the help of the other node $N_{j}, j \in\{1,2\}, i \neq j$, or node $N_{i}$ might not be able to achieve the performance $\gamma$ neither by its own nor by the help of node $N_{j}$. Therefore, if $\gamma$ is achievable, we provide the optimal operating mode of the system. If not, we consider that the optimal operating mode of the system is unfeasible.

Assume that the system operates in mixed $x$-n mode, where $x \in\{\mathrm{c}, \mathrm{r}\}$. As node $N_{i}$ may need the help of node $N_{j}$ to achieve $\gamma$, in $x$ mode node $N_{i}$ will operate as an $S_{2}$ while $N_{j}, j \neq i$ will operate as a cooperating or relay node depending on $x$. As stated earlier, when node $N_{i}$ operates as an $S_{2}$ in $x$ mode, its performance $\triangle_{i}$ will increase linearly over $n$. Therefore, because $\gamma$ is a value from 0 to 1 , the absolute difference between $\gamma$ and $\triangle_{i}$ will be convex over $n$ that increases linearly from both sides of its minimum, which is 0 . Finding the optimal $n$ and $x$ that minimize the absolute difference between $\gamma$ and $\triangle_{i}$ will provide the optimal operating mode of the system in which node $N_{i}$ achieves the performance $\gamma$. Under the constraints that $0 \leqslant n \leqslant N$ and $x \in\{\mathrm{c}, \mathrm{r}\}$, the problem can be formulated as

$$
\begin{array}{ll}
\min _{n, x} & \left|\gamma-\left(\triangle_{i}^{\mathrm{n}}+\frac{n\left(\triangle_{i}^{x}-\triangle_{i}^{\mathrm{n}}\right)}{N}\right)\right|, \\
\text { subject to } & x \in\{\mathrm{c}, \mathrm{r}\}, \\
& 0 \leqslant n \leqslant N .
\end{array}
$$

Define $n^{\mathrm{c}}$ and $n^{\mathrm{r}}$ as the values of $n$ that minimizes the cost function in (4.27) when $x=\mathrm{c}$ and when $x=\mathrm{r}$, respectively. Then by setting (4.27) equal to 0 and solving for $n$, the value of $n^{x}$ is

$$
n^{x}=\frac{\left(\gamma-\triangle_{i}^{\mathrm{n}}\right) N}{\triangle_{i}^{x}-\triangle_{i}^{\mathrm{n}}}
$$

From (4.28), $n^{x}$ will be in the range from $(0, N]$ if $\triangle_{i}^{\mathrm{n}}<\gamma \leqslant \triangle_{i}^{x}$, i.e., if node $N_{i}$ can achieve the performance $\gamma$ only with the help of node $N_{j}$ either by cooperation or relaying depending on $x$. $n^{x}$ will be higher than $N$ if $\gamma>\triangle_{i}^{x}$, i.e., $N_{i}$ cannot achieve the performance 
$\gamma$ neither by its own nor by the help of node $N_{j}$. Thus, due to the fact that $\triangle_{i}$ increases faster when $x=\mathrm{r}$ than when $x=$ c over $n$, we imply that $n^{\mathrm{r}}$ will definitely be in the range from $[0, N]$ if $n^{\mathrm{c}}$ is in the range from $[0, N]$, and not necessarily vice versa. In addition, we imply that $n^{\mathrm{c}}$ will definitely be higher than $N$ if $n^{\mathrm{r}}$ is higher than $N$, and not necessarily vice versa. This can be interpreted as that $\gamma$ will definitely be achieved when $x=\mathrm{r}$ if it is achieved when $x=\mathrm{c}$, and $\gamma$ will definitely not be achieved when $x=\mathrm{c}$ if it is not achieved when $x=$ r. Lastly, $n^{x}$ will be less than or equal to 0 if $\gamma \leqslant \triangle_{i}^{\mathrm{n}}$, which means that node $N_{i}$ can achieve the performance $\gamma$ on its own.

Based on $\triangle_{i}^{\mathrm{n}}$ and $n^{x}, x \in\{\mathrm{c}, \mathrm{r}\}$, we find the optimal solution $x^{\star}$ and $n^{\star}$ for (4.27). For a given $\gamma$, we first calculate $\triangle_{i}^{\mathrm{n}}$. If $\gamma \leqslant \triangle_{i}^{\mathrm{n}}$, i.e., node $N_{i}$ can achieve $\gamma$ by its own and $n^{\mathrm{r}}, n^{\mathrm{c}} \leqslant 0$, then the optimal solution is when $x^{\star}=\mathrm{r}$ and $n^{\star}=n^{\mathrm{r}}$. However, here node $N_{i}$ will operate as a relay to improve $N_{j}$ 's performance while achieving the performance $\gamma$. If $\gamma>\triangle_{i}^{\mathrm{n}}$, then we first calculate $n^{\mathrm{c}}$ and $n^{\mathrm{r}}$ using (4.28). If $n^{\mathrm{c}}, n^{\mathrm{r}} \in(0, N]$, i.e., $\gamma$ can be achieved when $x=\mathrm{c}$ and when $x=\mathrm{r}$, then there will be two optimal solutions. First is when $x^{\star}=\mathrm{c}$ and $n^{\star}=n^{\mathrm{c}}$ and second is when $x^{\star}=\mathrm{r}$ and $n^{\star}=n^{\mathrm{r}}$. We select the first solution because $N_{j}$ will achieve higher performance due to the fact that in relay mode $\triangle_{j}^{\mathrm{r}}=0$. If $n^{\mathrm{r}} \in(0, N]$ and $n^{\mathrm{c}} \notin(0, N]$, i.e., $\gamma$ can be achieved only when $x=\mathrm{r}$, then $x^{\star}=\mathrm{r}$ and $n^{\star}=n^{\mathrm{r}}$. If $n^{\mathrm{r}}, n^{\mathrm{c}}>N$, the optimal solution is considered unfeasible because $\gamma$ cannot be achieved even if node $N_{j}$ cooperates with or relays for node $N_{i}$ all the time blocks. The CS scheme, illustrated in Algorithm 4.3, summarizes the process of how to find the optimal solution for (4.27) when node $N_{1}$ needs to achieve the performance $\gamma$.

\subsection{Simulation Results}

We evaluate the performance of the proposed scheduling schemes in terms of the packet delivery ratio, assuming that the energy status in Algorithm 4.1 is known by feedback. For the simulations, we choose the system parameters shown in Figure 4.3, which shows the packet delivery ratios of $N_{1}$ and $N_{2}$ in noncooperation mode. We plot the packet delivery ratio versus energy harvesting rates $\overline{E_{1}}$ and $\overline{E_{2}}$ of $N_{1}$ and $N_{2}$, respectively. For better representation and analysis of the results, we consider that the energy harvesting processes 


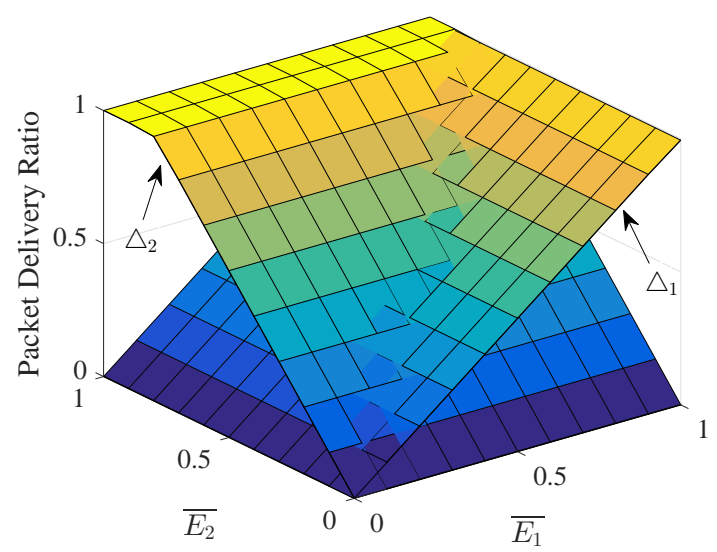

Figure 4.3: The packet delivery ratios of $N_{1}$ and $N_{2}$ in noncooperation mode. Parameters used: $q_{1}=0.5, q_{2}=0.4, \tilde{\delta}=1, \delta_{1}=\delta_{2}=2, \tilde{K}=1, K=100, N=5 \times 10^{5}$.

$E_{1}$ and $E_{2}$ are independent (uncorrelated) and $\tilde{K}=1$. So, $\overline{E_{1}}=p^{(1)}(1)$ and $\overline{E_{2}}=p^{(2)}(1)$, which are the probabilities of harvesting one unit of energy per time block by $N_{1}$ and $N_{2}$, respectively.

\subsubsection{Fairness}

We simulate the FS scheme summarized in Algorithm 4.2 using the penalty function given in Section 4.4.1 with three different values of $\alpha$. Figures (4.4), (4.5), and (4.6) illustrate the simulation results when $\alpha=1, \alpha=3$, and $\alpha=5$, respectively. $\alpha=1$ represents the case of absolute fairness, i.e., the case when the two nodes have to achieve the same performance. In this case, $n^{x}$ is not given by (4.26) because the cost function is not differentiable. So, we solve for it by setting the cost function to 0 without taking the derivative.

For each value of $\alpha$ as can be seen from Figures 4.4, 4.5, and 4.6, the FS scheme provides the optimal $x$ and $n$ that achieve fairness in the performance between the transmitting nodes. In the case of absolute fairness, we can notice from Figure 4.4 (c) that the two transmitting nodes achieve the same performance as expected. From Figures 4.4 (c), 4.5 (c), and 4.6 (c), it can be noticed that as $\alpha$ increases, the performance of each transmitting node gets closer to its performance in noncooperation mode shown in Figure 4.3. This implies that as $\alpha$ increases, the cooperation or relaying between the transmitting nodes decreases over the time blocks. Therefore, when one of the transmitting nodes achieves very high packet 


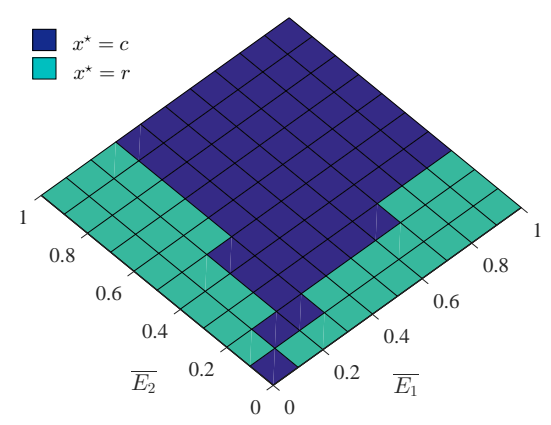

(a) Optimal $x$

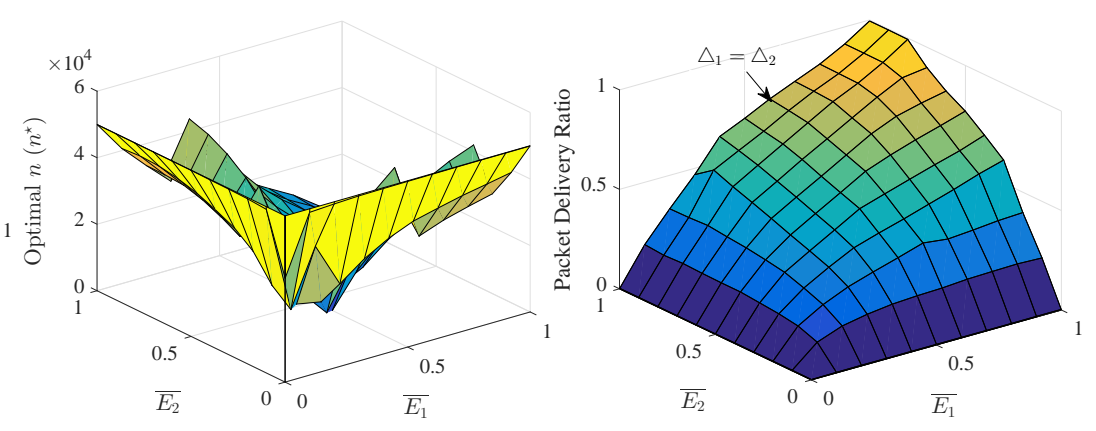

(b) Optimal $n$

(c) Optimal packet delivery ratio

Figure 4.4: Figures (a) and (b) illustrate the optimal $x$ and $n$ provided by the FS scheme when $\alpha=1$. Figure (c) shows the optimal performance of $N_{1}$ and $N_{2}$ by which fairness is considered achieved for the given penalty function.

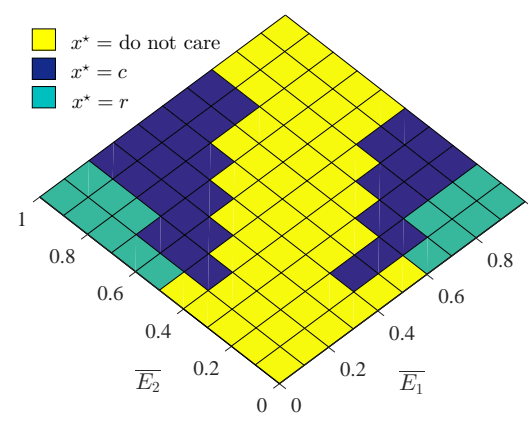

(a) Optimal $x$

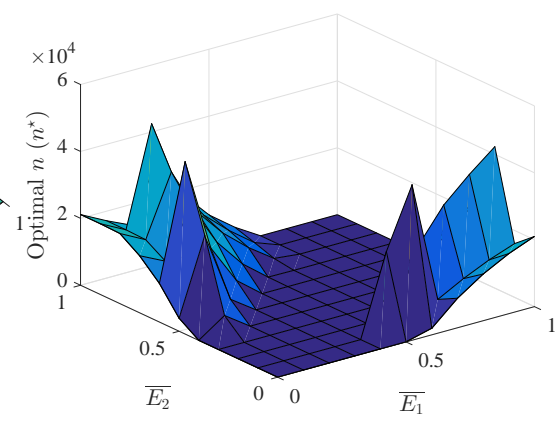

(b) Optimal $n$

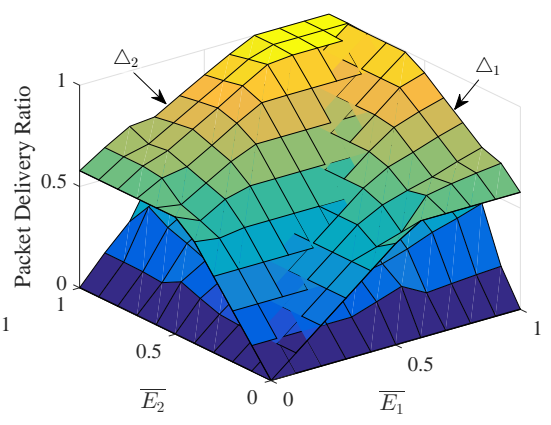

(c) Optimal packet delivery ratio

Figure 4.5: Figures (a) and (b) illustrate the optimal $x$ and $n$ provided by the FS scheme when $\alpha=3$. Figure (c) shows the optimal performance of $N_{1}$ and $N_{2}$ by which fairness is considered achieved for the given penalty function.

delivery ratio than the other in noncooperation mode, the value of $\alpha$ is recommended to be high. Hence, the node of higher performance does not cooperate with or relay for the other node over a long time, and thus it does not lose high performance for a slight improvement in the other node's performance, which is not considered in the adaptive scheme proposed in Chapter 3. 


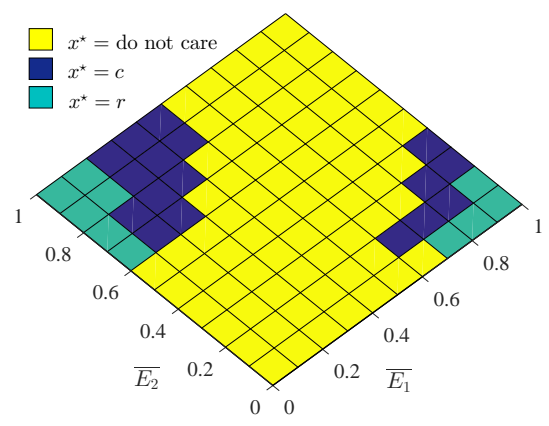

(a) Optimal $x$

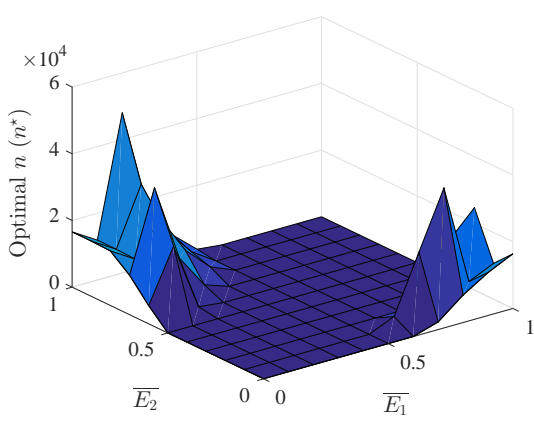

(b) Optimal $n$

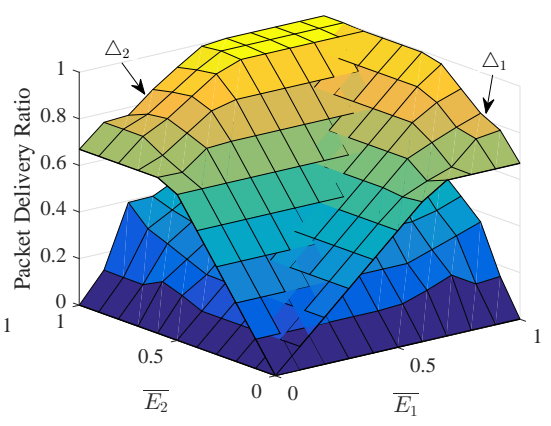

(c) Optimal packet delivery ratio

Figure 4.6: Figures (a) and (b) illustrate the optimal $x$ and $n$ provided by the FS scheme when $\alpha=5$. Figure (c) shows the optimal performance of $N_{1}$ and $N_{2}$ by which fairness is considered achieved for the given penalty function.

\subsubsection{Constrained Scheduling}

We simulate the CS scheme summarized in Algorithm 4.3 for the scenario when $N_{1}$ needs to report $70 \%$ of its total generated events to the destination, i.e., when $\gamma=0.7$. From the simulation results shown in Figure 4.7, we can see that the CS scheme provides the optimal $x$ and $n$ by which node $N_{1}$ achieves the performance $\gamma$. When $\overline{E_{1}} \geqslant 0.7$, node $N_{1}$ can achieve $\gamma$ by its own. Therefore, over this range, it operates as a relay for $N_{2}$ while achieving $\gamma$. When $\overline{E_{1}}<0.7$, node $N_{1}$ can achieve $\gamma$ over some values of $\overline{E_{2}}$ only by the help of node $N_{2}$ that is either by cooperating or relaying. So, over these values of $\overline{E_{2}}$, node $N_{1}$ operates as an $S_{2}$ to achieve $\gamma$ by the help of node $N_{2}$. For the remaining values of $\overline{E_{2}}$ when $\overline{E_{1}}<0.7$, node $N_{1}$ cannot achieve $\gamma$ neither by its own nor by the help of node $N_{2}$. Therefore, the optimal solution is unfeasible and the packet delivery ratios of the transmitting nodes are not plotted.

\subsection{Conclusions}

In this chapter, we consider a network of three nodes, two transmitting nodes and destination. We define three operating modes for the network that are combined appropriately to build two scheduling schemes. The first scheme is to ensure fairness in the performance between the two transmitting nodes while maximizing the sum of their packet delivery ra- 


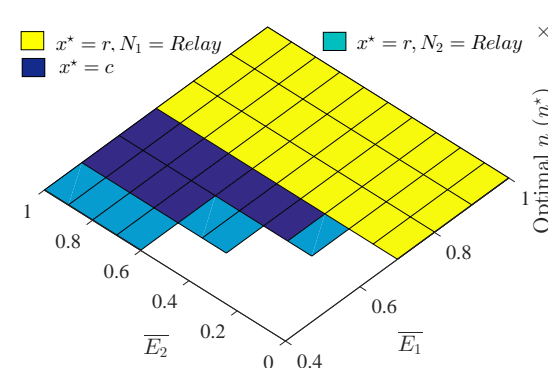

(a) Optimal $x$

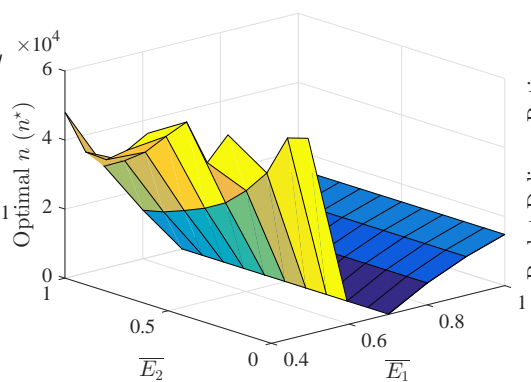

(b) Optimal $n$

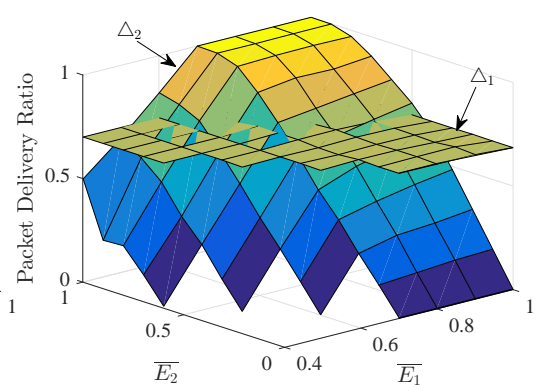

(c) Optimal packet delivery ratio

Figure 4.7: Figures (a) and (b) illustrate the optimal $x$ and $n$ provided by the CS scheme in order for node $N_{1}$ to achieve the performance $\gamma=0.7$. Figure (c) shows that node $N_{1}$ achieves the performance $\gamma$ over the values $\overline{E_{1}}$ and $\overline{E_{2}}$ where the system is feasible.

tios, and the second scheme is to constrain one of the transmitting nodes to achieve a certain performance. The FS scheme ensures fairness in the performance of the transmitting nodes based on a given penalty function that determines how much reduction in the performance of the cooperating or relay node can be tolerated. 


\section{Chapter 5}

\section{Scheduling with Energy Status Estimation}

\subsection{Introduction}

In the previous chapter, in part (a) of Algorithm 4.1 (Line 4 and Line 6), node $S_{2}$ needs the knowledge of the cooperating/relay node's energy status to make the decision on its usage. In this chapter, we introduce a statistical model to estimate the energy status of the cooperating/relay node at node $S_{2}$. As discussed in the previous chapters, the energy status of cooperating/relay node can be obtained by exchanging a few bits of information between nodes as in the the feedback scheme, or by comparing the energy battery level of the source by a threshold value as in the threshold-based scheme. However, in a practical fixed packet/frame format, as in IEEE 802.11, or in large networks with dynamic parameters, the overhead incurred or optimization of parameters may make estimation a better alternative.

In the proposed statistical model, node $S_{2}$ makes the decision on the energy status of the cooperating/relay node using a state information, which depends on the instantaneous transmission status of the cooperating/relay node. When the cooperating/relay node has a transmission, the state information includes the transmission status of the cooperating/relay node and its current energy battery level. As in [53], we assume that the current energy battery level of the cooperating/relay node is included in the header of its packet. So, whenever the cooperating/relay node has a transmission, the $S_{2}$ node receives only the 
header and reads the current energy battery level. When the cooperating/relay node has no transmission, the state information includes the transmission status of the cooperating/relay node and the relay transmission status of the $S_{2}$ node. We evaluate the performance of the statistical model by comparing it with the feedback scheme, which performs close to the optimal policy [53], as shown in Chapter 2.

\subsection{Cooperation Mode Statistical Model}

The cooperation mode scenario using the statistical model is similar to the cooperation mode scenario summarized in Algorithm 4.1 and Section 4.3.2. However, in the cooperation mode scenario using the statistical model, the $S_{2}$ node $N_{i}, i \in\{1,2\}$ in each time block makes the decision on its transmission action using the estimate energy status $\hat{A}_{j}^{\mathrm{c}}$ of the cooperating node $N_{j}, j \in\{1,2\}, j \neq i$. Thus, in the $n$-th time block, the direct transmission event $\tilde{d}_{1}^{\mathrm{c}}$ and the relay transmission event $\tilde{d}_{2}^{\mathrm{c}}$ of node $N_{i}$ become

$$
\begin{aligned}
& \tilde{d}_{1}^{\mathrm{c}}[n]= \begin{cases}1 & \text { if }\left(\mathcal{E}_{i}[n]=1 \cap L_{i}^{\mathrm{c}}[n] \geqslant \delta_{i} \cap \hat{A}_{j}^{\mathrm{c}}[n]=0\right) \\
0 & \text { otherwise, }\end{cases} \\
& \tilde{d}_{2}^{\mathrm{c}}[n]= \begin{cases}1 & \text { if }\left(\mathcal{E}_{i}[n]=1 \cap L_{i}^{\mathrm{c}}[n] \geqslant \tilde{\delta} \cap \hat{A}_{j}^{\mathrm{c}}[n]=1\right) \\
0 & \text { otherwise. }\end{cases}
\end{aligned}
$$

In each time block, the cooperating node $N_{j}$ senses the transmission action of the $S_{2}$ node $\tilde{d}_{1}^{\mathrm{c}}[n]$ and $\tilde{d}_{2}^{\mathrm{c}}[n]$ to decide on its transmission action. If $\tilde{d}_{2}^{\mathrm{c}}[n]=1$ is taken at $S_{2}$ based on a correct estimate of $A_{j}^{\mathrm{c}}[n]$, the transmission action at the cooperating node will be relay transmission. While if $\tilde{d}_{2}^{\mathrm{c}}[n]=1$ is taken based on a wrong estimate of $A_{j}^{\mathrm{c}}[n]$, the transmission action at the cooperating node will be no transmission, where in this case the $S_{2}$ event will be lost. If $\tilde{d}_{1}^{\mathrm{c}}[n]=1$, the cooperation node will take no transmission action regardless of its event and energy statuses due to Assumption 8, and to avoid interference. If $\tilde{d}_{2}^{\mathrm{c}}[n]=0$ and $\tilde{d}_{1}^{\mathrm{c}}[n]=0$, the transmission action at the cooperating node will be own-traf fic if it has an event generated and it is energized. Consequently, in the $n$-th time block, the own-traffic transmission event $\tilde{d}_{3}^{\mathrm{c}}[n]$ and the relay transmission event $\tilde{d}_{4}^{\mathrm{c}}[n]$ of the cooperating 
node are

$$
\begin{gathered}
\tilde{d}_{3}^{\mathrm{c}}[n]= \begin{cases}1 & \text { if }\left(\tilde{d}_{1}^{\mathrm{c}}[n]=0 \cap \tilde{d}_{2}^{\mathrm{c}}[n]=0 \cap \mathcal{E}_{j}[n]=1 \cap L_{j}^{\mathrm{c}}[n] \geqslant \delta_{j}\right) \\
0 & \text { otherwise, }\end{cases} \\
\tilde{d}_{4}^{\mathrm{c}}[n]= \begin{cases}1 & \text { if }\left(\tilde{d}_{2}^{\mathrm{c}}[n]=1 \cap L_{j}^{\mathrm{c}}[n] \geqslant \delta_{j}\right) \\
0 & \text { otherwise. }\end{cases}
\end{gathered}
$$

Using $\tilde{d}_{1}^{\mathrm{c}}[n], \tilde{d}_{2}^{\mathrm{c}}[n], \tilde{d}_{3}^{\mathrm{c}}[n]$, and $\tilde{d}_{4}^{\mathrm{c}}[n]$, in the next time block the energy battery level $L_{i}^{\mathrm{c}}$ of the $S_{2}$ node $N_{i}$ is

$$
L_{i}^{\mathrm{c}}[n+1]=\min \left\{L_{i}^{\mathrm{c}}[n]+E_{i}[n]-\tilde{\delta} \tilde{d}_{2}^{\mathrm{c}}[n]-\delta_{i} \tilde{d}_{1}^{\mathrm{c}}[n], K\right\}
$$

and the energy battery level $L_{j}^{\mathrm{c}}$ of the cooperating node $N_{j}$ in the next time block is

$$
L_{j}^{\mathrm{c}}[n+1]=\min \left\{L_{j}^{\mathrm{c}}[n]+E_{j}[n]-\delta_{j} \tilde{d}^{\mathrm{c}}[n], K\right\},
$$

where $\tilde{d}^{\mathrm{c}}[n]=\tilde{d}_{4}^{\mathrm{c}}[n]+\tilde{d}_{3}^{\mathrm{c}}[n]$ is either 0 or 1 since $\left(\tilde{d}_{4}^{\mathrm{c}}[n]=1 \cap \tilde{d}_{3}^{\mathrm{c}}[n]=1\right)=\emptyset \cdot \tilde{d}^{\mathrm{c}}[n]=1$ means that the cooperating node has either a relay or an own traffic transmission in the $n$-th time block, and $\tilde{d}^{\mathrm{c}}[n]=0$ means that the cooperating node has no transmission.

Now after deriving its model in (5.6), the energy battery level $L_{j}^{\mathrm{c}}[n+1]$ is estimated at $S_{2}$ using the state information of the current time block $\mathcal{X}[n]$. One of the pieces of information included in $\mathcal{X}[n]$ is $E_{i}[n]$ which is correlated with $E_{j}[n]$. The rest of the information depends on the transmission status $\tilde{d}^{\mathrm{c}}[n]$ of node $N_{j}$, which is considered known at the $S_{2}$ node by sensing the link between $N_{j}$ and the destination in each time block. If $\tilde{d}^{\mathrm{c}}[n]=1$, the rest of the information includes: a) the transmission status of the cooperating node $\tilde{d}^{\mathrm{c}}[n]=1$, and b) the current energy battery level of the cooperating node $L_{j}^{\mathrm{c}}[n]=u, u \in\{0,1, \ldots, K\}$, $u \geqslant \delta_{j}$. As stated earlier, we assume that $L_{j}^{\mathrm{c}}[n]$ is included in the header of the transmitted packet. Whenever the cooperating node transmits, the $S_{2}$ node receives only the header and reads $L_{j}^{\mathrm{c}}[n]$. If $\tilde{d}^{\mathrm{c}}[n]=0$, the rest of the information includes: a) the transmission status of the cooperating node $\tilde{d}^{c}[n]=0$, and b) the relay transmission action of the $S_{2}$ node $\tilde{d}_{2}^{c}[n]=b \in\{0,1\}$. The state information can be summarized as

$$
\mathcal{X}[n]=\left\{\begin{array}{lll}
\left\{E_{i}[n], \tilde{d}^{\mathrm{c}}[n]=1, L_{j}^{\mathrm{c}}[n]=u\right\} & \text { if }\left(\tilde{d}^{\mathrm{c}}[n]=1\right) & \text { Case } 1 \\
\left\{E_{i}[n], \tilde{d}^{\mathrm{c}}[n]=0, \tilde{d}_{2}^{\mathrm{c}}[n]=b\right\} & \text { if }\left(\tilde{d}^{\mathrm{c}}[n]=0\right) & \text { Case } 2
\end{array}\right.
$$


Given the state information $\mathcal{X}[n-1]$, the maximum likelihood estimator of $L_{j}^{\mathrm{c}}[n]$ at $S_{2}$ is

$$
\hat{L}_{j}^{\mathrm{c}}[n]=\underset{0 \leqslant k \leqslant K}{\arg \max } \operatorname{Pr}\left(L_{j}^{\mathrm{c}}[n]=k \mid \mathcal{X}[n-1]\right) .
$$

Using $\hat{L}_{j}^{\mathrm{c}}[n]$, the estimate energy status of the $n$-th time block is

$$
\hat{A}_{j}[n]= \begin{cases}1 & \text { if } \hat{L}_{j}^{\mathrm{c}}[n] \geqslant \delta_{j} \\ 0 & \text { otherwise }\end{cases}
$$

Depending on the state information $\mathcal{X}[n-1]$, the probability $\operatorname{Pr}\left(L_{j}^{\mathrm{c}}[n]=k \mid \mathcal{X}[n-1]\right)$ in (5.8) is calculated as follows.

Case 1: when $\mathcal{X}[n-1]=\left\{E_{i}[n-1], \tilde{d}^{\mathrm{c}}[n-1]=1, L_{j}^{\mathrm{c}}[n-1]=u\right\}$. Let $\mathcal{L}_{j}^{\mathrm{c}}[n]$ be a variable that is defined as

$$
\mathcal{L}_{j}^{\mathrm{c}}[n]=L_{j}^{\mathrm{c}}[n-1]+E_{j}[n-1]-\delta_{j} \tilde{d}^{\mathrm{c}}[n-1] .
$$

Because $\tilde{d}^{\mathrm{c}}[n-1]=1, \mathcal{L}_{j}^{\mathrm{c}}[n]$ can be a value from 0 to $K+\tilde{K}-\delta_{j}$. Using (5.10) and because $E_{j}$ and $E_{i}$ are correlated, $\operatorname{Pr}\left(\mathcal{L}_{j}^{\mathrm{c}}[n]=v \mid \mathcal{X}[n-1]\right), v \in\left\{0,1, \ldots, K+\tilde{K}-\delta_{j}\right\}$ is

$$
\operatorname{Pr}\left(\mathcal{L}_{j}^{\mathrm{c}}[n]=v \mid \mathcal{X}[n-1]\right)=\operatorname{Pr}\left(E_{j}[n-1]=\tilde{k} \mid E_{i}[n-1]\right)=\frac{\operatorname{Pr}\left(\tilde{k}, E_{i}[n-1]\right)}{p^{(i)}\left(E_{i}[n-1]\right)},
$$

where $\tilde{k}=v-u+\delta_{j}$ and $\operatorname{Pr}\left(\tilde{k}, E_{i}[n]\right)=0 \forall \tilde{k} \notin[0, \tilde{K}]$. Using $\operatorname{Pr}\left(\mathcal{L}_{j}^{\mathrm{c}}[n]=v \mid \mathcal{X}[n-1]\right)$, we can calculate $\operatorname{Pr}\left(L_{j}^{\mathrm{c}}[n]=k \mid \mathcal{X}[n-1]\right) \forall 0 \leqslant k \leqslant K$ as follows. If $\tilde{K}<\delta_{j}$,

$$
\operatorname{Pr}\left(L_{j}^{\mathrm{c}}[n]=k \mid \mathcal{X}[n-1]\right)= \begin{cases}\operatorname{Pr}\left(\mathcal{L}_{j}^{\mathrm{c}}[n]=k \mid \mathcal{X}[n-1]\right) & \text { if } 0 \leqslant k \leqslant K+\tilde{K}-\delta_{j}, \\ 0 & \text { otherwise }\end{cases}
$$

and if $\tilde{K} \geqslant \delta_{j}$

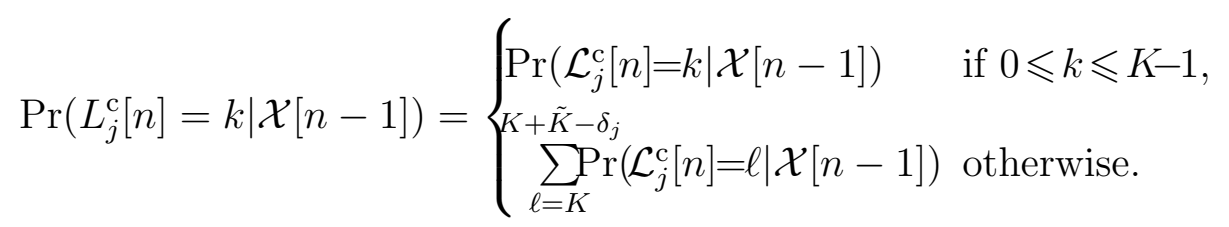

Case 2: when $\mathcal{X}[n-1]=\left\{E_{i}[n-1], \tilde{d}^{\mathrm{c}}[n-1]=0, \tilde{d}_{2}^{\mathrm{c}}[n-1]=b\right\}$. Because $\tilde{d}^{\mathrm{c}}[n-1]=0$, $\mathcal{L}_{j}^{\mathrm{c}}[n]$ in $(5.10)$ becomes

$$
\mathcal{L}_{j}^{\mathrm{c}}[n]=L_{j}^{\mathrm{c}}[n-1]+E_{j}[n-1] .
$$


Here, $\mathcal{L}_{j}^{\mathrm{c}}[n]$ can be a value from 0 to $K+\tilde{K}$. Because $E_{j}$ and $E_{i}$ are correlated and $L_{j}^{\mathrm{c}}$ and $E_{j}$ in the same time block are independent, the distribution of $\left(\mathcal{L}_{j}^{\mathrm{c}}[n] \mid \mathcal{X}[n-1]\right)$ is the convolution of $\operatorname{Pr}\left(L_{j}^{\mathrm{c}}[n-1]\right)$ and $\operatorname{Pr}\left(E_{j}[n-1] \mid E_{i}[n-1]\right)$. Thus, $\operatorname{Pr}\left(\mathcal{L}_{j}^{\mathrm{c}}[n]=v \mid \mathcal{X}[n-1]\right)$, $v \in\{0,1, \ldots, K+\tilde{K}\}$ is calculated as

$$
\operatorname{Pr}\left(\mathcal{L}_{j}^{\mathrm{c}}[n]=v \mid \mathcal{X}[n-1]\right)=\sum_{\tilde{k}=0}^{\tilde{K}} \operatorname{Pr}\left(L_{j}^{\mathrm{c}}[n-1]=v-\tilde{k}\right) \frac{\operatorname{Pr}\left(\tilde{k}, E_{i}[n-1]\right)}{p^{(i)}\left(E_{i}[n-1]\right)} .
$$

The distribution of $L_{j}^{\mathrm{c}}[n-1]$ in (5.13) is the same as the distribution of $L_{j}^{\mathrm{c}}[n-1]$ that was calculated in the previous time block given the state information $\mathcal{X}(n-2)$, i.e., $\operatorname{Pr}\left(L_{j}^{\mathrm{c}}[n-1]=\right.$ $u)=\operatorname{Pr}\left(L_{j}^{\mathrm{c}}[n-1]=u \mid \mathcal{X}(n-2)\right), u \in\{0,1, \ldots, K\}$. However, if $b=1$, i.e., $A^{\mathrm{c}}[n-1]$ was estimated wrong and $L_{j}^{\mathrm{c}}[n-1]$ should be a value that is less than $\delta_{j}$ since $\tilde{d}^{\mathrm{c}}[n-1]=0$, then we weight the probabilities $\operatorname{Pr}\left(L_{j}^{\mathrm{c}}[n-1]=u \mid \mathcal{X}(n-2)\right), \delta_{j} \leqslant u \leqslant K$ on the probabilities $\operatorname{Pr}\left(L_{j}^{\mathrm{c}}[n-1]=u \mid \mathcal{X}(n-2)\right), 0 \leqslant u<\delta_{j}$ as $\operatorname{Pr}\left(L_{j}^{\mathrm{c}}[n-1]=u\right)= \begin{cases}\operatorname{Pr}\left(L_{j}^{\mathrm{c}}[n-1]=u \mid \mathcal{X}(n-2)\right)+\left(\sum_{l=\delta_{j}}^{K} \operatorname{Pr}\left(L_{j}^{\mathrm{c}}[n-1]=l \mid \mathcal{X}(n-2)\right)\right) / \delta_{j} & \text { if } 0 \leqslant u \leqslant \delta_{j}-1, \\ 0 & \text { otherwise }\end{cases}$

Using $\operatorname{Pr}\left(\mathcal{L}_{j}^{\mathrm{c}}[n]=v \mid \mathcal{X}[n-1]\right)$, we can calculate $\operatorname{Pr}\left(L_{j}^{\mathrm{c}}[n]=k \mid \mathcal{X}[n-1]\right) \forall 0 \leqslant k \leqslant K$ as follows.

$$
\operatorname{Pr}\left(L_{j}^{\mathrm{c}}[n]=k \mid \mathcal{X}[n-1)= \begin{cases}\operatorname{Pr}\left(\mathcal{L}_{j}^{\mathrm{c}}[n]=k \mid \mathcal{X}[n-1]\right) & \text { if } 0 \leqslant k \leqslant K-1, \\ \sum_{\ell=K}^{K+\tilde{K}} \operatorname{Pr}\left(\mathcal{L}_{j}^{\mathrm{c}}[n]=\ell \mid \mathcal{X}[n-1]\right) & \text { otherwise }\end{cases}\right.
$$

Note that in order for this estimation model to work, the knowledge of the initial energy battery level of the cooperating node is needed at the $S_{2}$ node. Therefore, we assume that the initial energy battery level of the cooperating node is transmitted to the $S_{2}$ node in the first time block. This is equivalent to setting $\tilde{d}^{c}[0]=1$. Using the estimation model, the packet delivery ratio $\triangle_{i}^{\mathrm{c}}$ of $S_{2}$ is

$$
\begin{gathered}
\triangle_{i}^{\mathrm{c}}=\frac{\left[\operatorname{Pr}\left(\tilde{d}_{1}^{\mathrm{c}}=1\right)+\operatorname{Pr}\left(\tilde{d}_{2}^{\mathrm{c}}=1 \cap \tilde{d}_{4}^{\mathrm{c}}=1\right)\right] N}{\operatorname{Pr}\left(\mathcal{E}_{i}=1\right) N}, \\
\triangle_{i}^{\mathrm{c}}=\operatorname{Pr}\left(L_{i}^{\mathrm{c}} \geqslant \delta_{i} \cap \hat{L}_{j}^{\mathrm{c}}<\delta_{j}\right)+\operatorname{Pr}\left(L_{i}^{\mathrm{c}} \geqslant \tilde{\delta} \cap L_{j}^{\mathrm{c}} \geqslant \delta_{j} \cap \hat{L}_{j}^{\mathrm{c}} \geqslant \delta_{j}\right),
\end{gathered}
$$


and the packet delivery ratio $\triangle_{j}^{c}$ of the cooperating node is

$$
\begin{gathered}
\triangle_{j}^{\mathrm{c}}=\frac{\operatorname{Pr}\left(\tilde{d}_{3}^{\mathrm{c}}=1\right) N}{\operatorname{Pr}\left(\mathcal{E}_{i}=1\right) N}, \\
\triangle_{j}^{\mathrm{c}}=\left(1-q_{i}\right) \operatorname{Pr}\left(L_{j}^{\mathrm{c}} \geqslant \delta_{j}\right)+\operatorname{Pr}\left(L_{i}^{\mathrm{c}}<\tilde{\delta} \cap L_{j}^{\mathrm{c}} \geqslant \delta_{j}\right)+ \\
\operatorname{Pr}\left(\tilde{\delta} \leqslant L_{i}^{\mathrm{c}}<\delta_{i} \cap L_{j}^{\mathrm{c}} \geqslant \delta_{j} \cap \hat{L}_{j}^{\mathrm{c}}<\delta_{j}\right)-\left(1-q_{i}\right)\left[\operatorname{Pr}\left(L_{i}^{\mathrm{c}}<\tilde{\delta} \cap L_{j}^{\mathrm{c}} \geqslant \delta_{j}\right)+\right. \\
\left.\operatorname{Pr}\left(\tilde{\delta} \leqslant L_{i}^{\mathrm{c}}<\delta_{i} \cap L_{j}^{\mathrm{c}} \geqslant \delta_{j} \cap \hat{L}_{j}^{\mathrm{c}}<\delta_{j}\right)\right] .
\end{gathered}
$$

Here, there is no closed form expression for the joint distribution of $L_{i}^{\mathrm{c}}, L_{j}^{\mathrm{c}}$, and $\hat{L}_{j}^{\mathrm{c}}$. Therefore, we use Monte Carlo technique to find the packet delivery ratios in (5.15) and (5.17).

\subsection{Relay Mode Statistical Model}

As mentioned in Section 4.3.3, the difference between cooperation and relay modes is that in relay mode node $N_{j}$ operates as a relay, which has no own transmissions. Therefore, the cooperation mode statistical model detailed in Section 5.2 can represent the relay mode statistical model if the superscript $\mathrm{c}$ is replaced by $\mathrm{r}$ and $\tilde{d}_{3}^{\mathrm{r}}$ is set to 0 . Hence, the packet delivery ratio $\triangle_{i}^{\mathrm{r}}$ of $S_{2}$ is

$$
\triangle_{i}^{\mathrm{r}}=\operatorname{Pr}\left(L_{i}^{\mathrm{r}} \geqslant \delta_{i} \cap \hat{L}_{j}^{\mathrm{r}}<\delta_{j}\right)+\operatorname{Pr}\left(L_{i}^{\mathrm{r}} \geqslant \tilde{\delta} \cap L_{j}^{\mathrm{r}} \geqslant \delta_{j} \cap \hat{L}_{j}^{\mathrm{r}} \geqslant \delta_{j}\right)
$$

and the packet delivery ratio of the relay node is $\triangle_{j}^{\mathrm{r}}=0$.

\subsection{Simulation Results}

We simulate the cooperation and relay modes in Sections 4.3.2 and 4.3.3 using the statistical models and the feedback scheme. As stated in Chapter 1, the feedback scheme provides the upper-bound performance as it performs close to the optimal policy. In the simulations, we use the system parameters shown in Figure 4.3. We plot the performance in terms of the packet delivery ratio versus energy harvesting rates $\overline{E_{1}}$ and $\overline{E_{2}}$ of $N_{1}$ and $N_{2}$, respectively. 


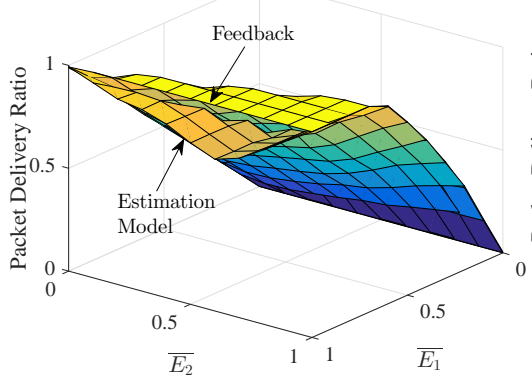

(a) Cooperation mode

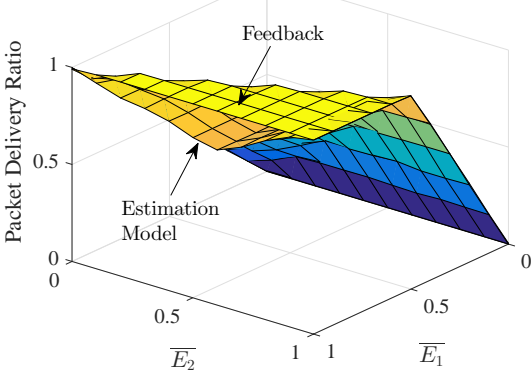

(b) Relay mode

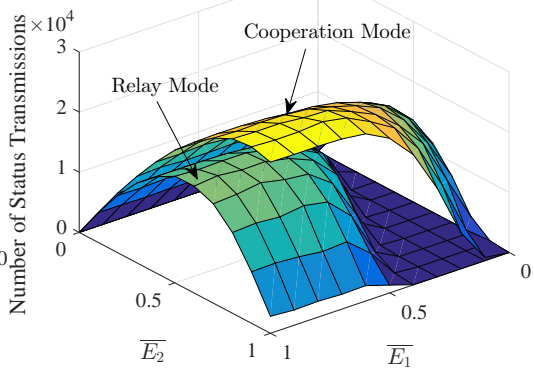

(c) Number of energy status transmissions of $N_{2}$

Figure 5.1: Figures (a) and (b) show the performance of $N_{1}$ in the cooperation and relay modes using the statistical models compared with the feedback scheme. Figure (c) shows the number of energy status transmissions of $N_{2}$ over the $N$ time blocks.

We simulate the case when the energy harvesting processes $E_{1}$ and $E_{2}$ are independent (uncorrelated) and $\tilde{K}=1$. So, $\overline{E_{1}}=p^{(1)}(1)$ and $\overline{E_{2}}=p^{(2)}(1)$, which are the probabilities of harvesting one unit of energy per time block by $N_{1}$ and $N_{2}$, respectively. We also simulate the case when $E_{1}$ and $E_{2}$ are correlated. In this case, we plot the packet delivery ratio versus correlation coefficient $\rho$ assuming that $\tilde{K}=1$ and $p^{(1)}(1)=p^{(2)}(1)=p$. Given $\rho$ and $p$, the joint probability $\operatorname{Pr}\left(E_{1}=1, E_{2}=1\right)=p^{2}+\rho p(1-p)$. Using $p$ and $\operatorname{Pr}\left(E_{1}=1, E_{2}=1\right)$, we calculate the rest of the joint probabilities $P\left(E_{1}=0, E_{2}=0\right), P\left(E_{1}=1, E_{2}=0\right)$, and $P\left(E_{1}=0, E_{2}=1\right)$. Finally, we simulate the FS and CS schemes proposed in the previous chapter using the statistical models for the case when $E_{1}$ and $E_{2}$ are independent.

\subsubsection{Cooperation and relay modes (independent case)}

We simulate the cooperation and relay modes using the statistical models when $E_{1}$ and $E_{2}$ are independent, where in both modes $N_{1}$ operates as an $S_{2}$ and needs to estimate the energy status of $N_{2}$. Figures 5.1 (a) and (b) illustrate the packet delivery ratio of node $N_{1}$ for the cooperation and relay modes, respectively. Figure 5.1 (c) shows the total number of energy status transmitted from $N_{2}$ to $N_{1}$ in the feedback scheme. From Figures 5.1 (a) and (b) we can notice that in both modes when $\overline{E_{1}}$ is high, the gap between the statistical model and feedback scheme increases as $\overline{E_{2}}$ movies towards 0.6 , at which the uncertainty of 


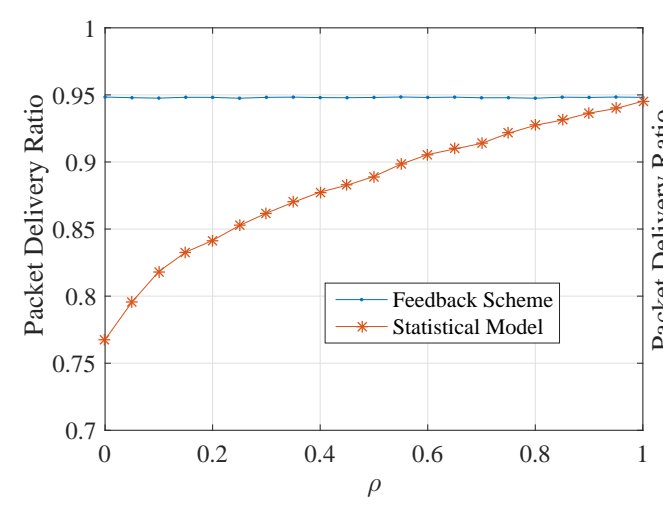

(a) Cooperation mode

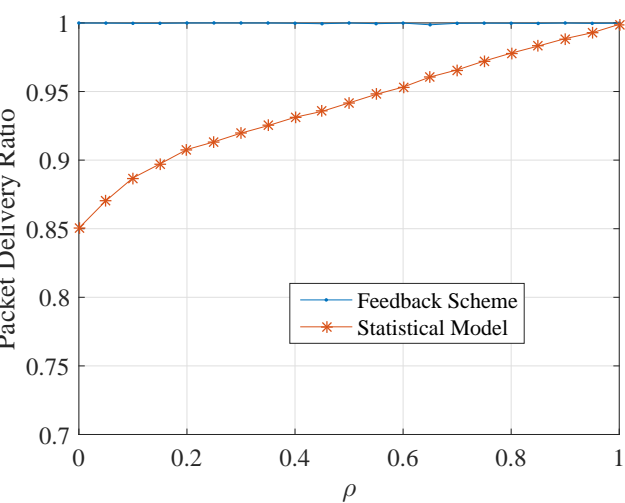

(b) Relay mode

Figure 5.2: Figures (a) and (b) show the packet delivery ratio of $N_{1}$ versus correlation coefficient $\rho$ for the cooperation and relay modes, respectively, using the statistical models compared with the feedback scheme.

the energy status of node $N_{2}$ is maximized. The fact that the uncertainty is maximized at 0.6 can also be noticed from the feedback scheme in Figure 5.1 (c) if the probability that the energy status changes in each time block is defined as the number of status transmissions over the total number of time blocks. From Figure 5.1 (c), we can observe that the number of energy status transmissions is 0 over some values of $\overline{E_{1}}$ and $\overline{E_{2}}$, which means that over these values the energy status of $N_{2}$ is constant or deterministic. Therefore, over these values, the statistical model and the feedback scheme perform the same. Hence, we conclude that when the energy status of the cooperating/relay node is deterministic, the feedback scheme will be more energy efficient than the statistical model. Otherwise, the statistical model will be more energy efficient as the energy status transmissions of $N_{2}$ could consume a significant amount of energy, especially when using protocols with fixed frame/packet format.

\subsubsection{Cooperation and relay modes (correlated case)}

Here, we simulate the cooperation and relay modes when the energy harvesting processes $E_{1}$ and $E_{2}$ are correlated, assuming that $p=0.7$ and $N_{1}$ operates as an $S_{2}$ in both modes. Figures 5.2 (a) and (b) show the simulation results in terms of the packet delivery ratio versus correlation coefficient $\rho$ for the cooperation and relay modes, respectively. It can be 


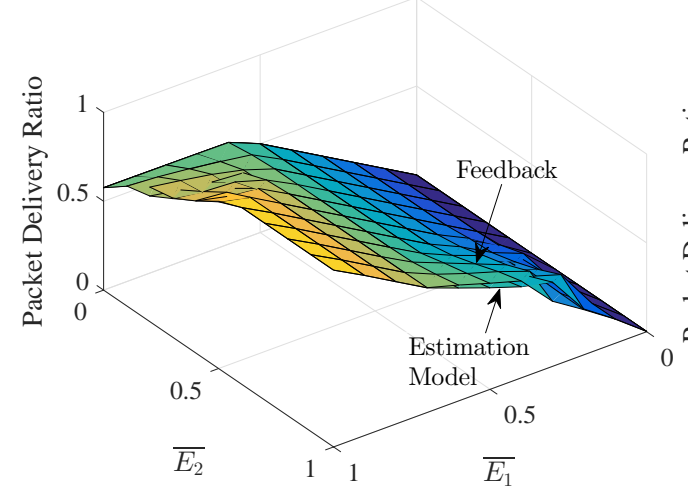

(a) Packet delivery ratio of $N_{1}$

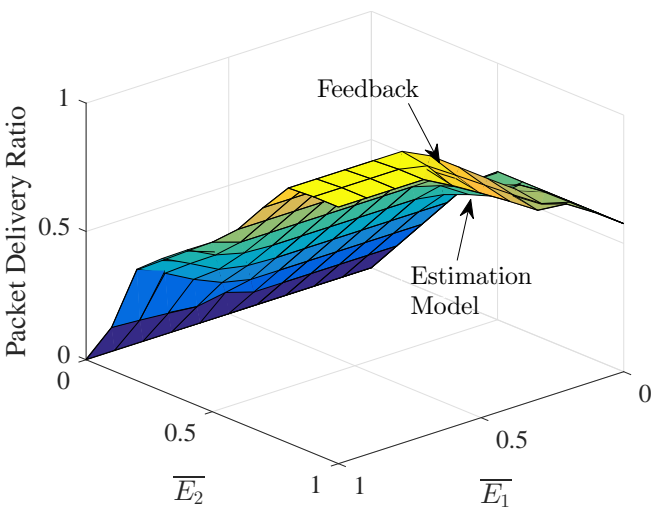

(b) Packet delivery ratio of $N_{2}$

Figure 5.3: Figures (a) and (b) show the packet delivery ratios of $N_{1}$ and $N_{2}$, respectively, for the FS scheme when using the statistical models compared with the feedback scheme. We used the same penalty function in Section VI (A) with $\alpha=3$.

noticed that the performance of the feedback scheme is not affected by $\rho$ because the decision is made based on the actual energy status of node $N_{2}$. In the statistical model, on the other hand, the performance is affected by $\rho$, where it increases as the correlation between $E_{1}$ and $E_{2}$ gets stronger until it reaches the performance of the feedback scheme at $\rho=1$, where the energy status of $N_{2}$ becomes actual at $N_{1}$.

\subsubsection{FS and CS schemes}

We simulate the FS and CS schemes for the same scenarios in Sections 4.5.1 and 4.5.2, respectively, using the statistical models. In the FS scheme, we only simulate the scenario when $\alpha=3$, and we calculate the value of $n^{x}$ by (4.26) using the packet delivery ratios derived in Sections 5.2 and 5.3. Due to the imperfect estimation, $n^{x}$ sometimes comes out as a complex value in the cases when the energy harvesting rate of $S_{2}$ is much lower than its event generation rate. In these cases, we just set $n^{x}=0$ as it is supposed to be.

Figures 5.3 and 5.4 illustrate the simulation results for the FS and CS schemes, respectively. For the FS scheme, Figure 5.3 shows that the statistical model performs either the same or very close to the feedback scheme. This is because whenever the system operates in the cooperation or relay mode, i.e, whenever $x^{\star}$ is decided to be either $\mathrm{c}$ or $\mathrm{r}$ in the FS 


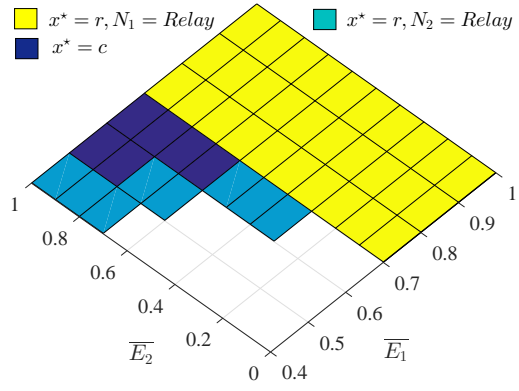

(a) Optimal $x$

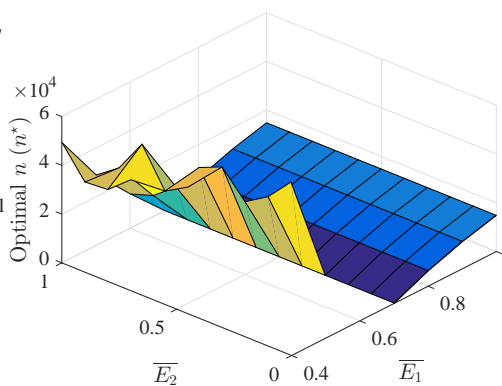

(b) Optimal $n$

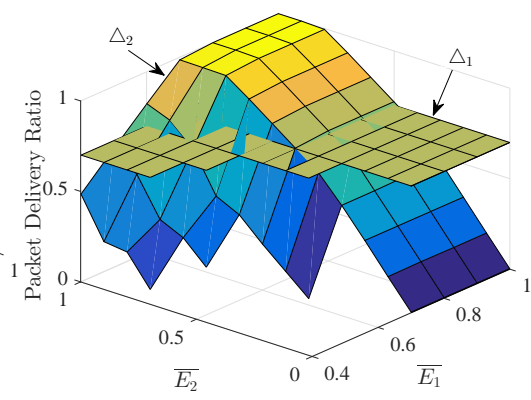

(c) Packet delivery ratios of $N_{1}$ and $N_{2}$

Figure 5.4: Figures (a) and (b) illustrate the optimal $x$ and $n$ provided by the CS scheme using the statistical models in order for node $N_{1}$ to achieve the performance $\gamma=0.7$. Figure (c) shows that node $N_{1}$ achieves the performance $\gamma$ over the values $\overline{E_{1}}$ and $\overline{E_{2}}$ where the system is feasible.

algorithm, the system operates over the ranges of $\overline{E_{1}}$ and $\overline{E_{2}}$ where the statistical model and the feedback scheme perform the same or close to each other. As for the CS scheme when using the statistical model, Figure 5.4 shows that the range of $\overline{E_{1}}$ and $\overline{E_{2}}$ over which $N_{1}$ achieves the performance $\gamma$ by its own stays the same, and the range over which $N_{1}$ achieves $\gamma$ by the help of node $N_{2}$ shrinks compared to the feedback scheme shown in Figure 4.7. This is expected because when $N_{1}$ achieves the performance $\gamma$ by its own, it operates as a relay for $N_{2}$ over $n^{\mathrm{r}}$ time blocks. After that, it operates as an $S_{1}$ to achieve $\gamma$ over the rest of the time blocks. From (4.28) and because $\triangle_{1}^{\mathrm{r}}=0, n^{\mathrm{r}}$ will be the same in both the statistical model and feedback scheme, which can also be seen from Figures 5.4 (b) and 4.7 (b). The same value of $n^{\mathrm{r}}$ results in the same range of $\overline{E_{1}}$ and $\overline{E_{2}}$ over which $N_{1}$ achieves $\gamma$ by its own. In contrast, when $N_{1}$ achieves $\gamma$ by the help of node $N_{2}$, it operates as an $S_{2}$ over $n^{x}, x \in\{\mathrm{c}, \mathrm{r}\}$ time blocks and then it operates as an $S_{1}$. Here, $\triangle_{1}^{x}, x \in\{\mathrm{c}, \mathrm{r}\}$ in (4.26) is calculated using the statistical model, which results in a lower $n^{x}$ compared to the feedback scheme due to the imperfect estimation. Thus, the range over which $N_{1}$ can achieve $\gamma$ only by the help of $N_{2}$ shrinks compared to the feedback scheme. 


\subsection{Conclusions}

In this chapter, we propose a statistical model that can be used to estimate the energy status of the cooperating/relay node at the source. In the scenarios when the energy status of the cooperating/relay node is certain, i.e., it is either energized or not over the $N$ time blocks, it will be more energy efficient to use the feedback scheme where in these scenarios there will be 0 overhead. Otherwise, it will be more energy efficient to use the statistical model because overhead could consume a significant amount of energy, especially, when using protocols with fixed frame/packet format. 


\section{Chapter 6}

\section{Scheduling for Throughput Maximization}

\subsection{Introduction}

In this chapter, we address the problem of scheduling for throughput maximization in a wireless energy harvesting uplink. For fairness, we assume that each node's throughput cannot drop below what it achieves by direct transmission to the base station. We tackle this problem by assigning a role to each node in the network, that is either a cooperating node, a Source type 1 (type $\left.S_{1}\right)$, or a Source type $2\left(S_{2}\right)$. The role assignments of the nodes are optimally done based on the energy harvesting rate of each node compared to its energy depletion rate. Such that, a node of a higher energy harvesting rate is assigned to be a cooperating node of some type $S_{2}$ nodes, some nodes of lower energy harvesting rates, if (1) the total number of events that are successfully transmitted from that cooperating node to the base station, including its type $S_{2}$ nodes' events, is higher than or equal to the total number of events that are successfully transmitted from the cooperating node and its type $S_{2}$ nodes when each transmits its events on its own to the base station, and (2) that cooperating node can forward more events for each one of its type $S_{2}$ nodes than any other cooperating node in the network. If a node can neither be a cooperating node nor a type $S_{2}$ node, it will be assigned as a type $S_{1}$ node, which transmits its events on its own to the base station.

We first analyze the problem for the scenario when the transmit power between the nodes

Portion of this chapter appears in: A. M. A. Ammar, Y. P. Fallah and D. Reynolds, "Throughput in an Energy Harvesting Wireless Uplink," in IEEE Sensors Journal, vol. 18, no. 6, pp. 2617-2626, March 15, 2018. 


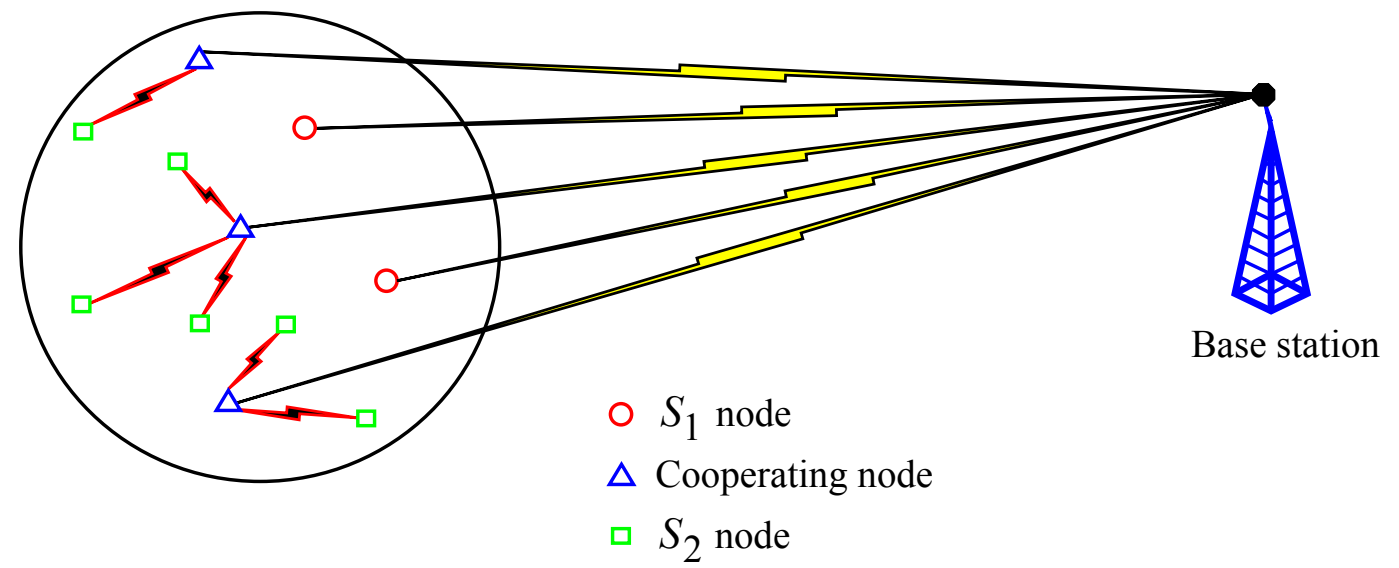

Figure 6.1: A network consists three categories of sensor nodes; type $S_{1}$ node which transmits directly to the base station, type $S_{2}$ node which transmits to the base station through another node called cooperating node, and cooperating node which transmits its own events as well as it forwards some type $S_{2}$ nodes' events to the base station.

is the same. After that, we generalize it for the scenario when the transmit power between the nodes is variable. We first derive the data collection throughput of the network as the summation of the successful transmission probabilities of its sensor nodes to the base station. We then propose a centralized algorithm that maximizes the throughput by optimally assigning a role to each node. The algorithm is run at the base station after receiving the energy harvesting and event generation rates of each node, and then a message that contains the role of each node is broadcasted to the network. We also develop a distributed algorithm for use in networks when central control is not feasible, e.g., when overhead must be kept low or when the base station is also energy-constrained. The results show that the proposed algorithms maximize the throughput with less computation complexity than the brute force approach, where all the possible combinations of nodes' roles that are applicable to the system model are examined. The results also show that our approach, compared with cooperative communication, maximizes the overall throughput of the network such that no node's throughput is adversely affected. Note that, the role assignments of the nodes are quasi-static, in that changes in the network topology or energy harvesting parameters requires that the algorithm run again.

One practical application is bridge health monitoring [66]-[68], where nodes are usually 
powered by sunlight and/or vibration. Hence, the nodes that are exposed to sunlight or located along the span are more likely to have a higher energy harvesting rate than their energy depletion rate. Thus, these nodes can relay transmissions of the nodes that are located inside the bridge or away from the span to the base station in order to maximize the network throughput.

\subsection{System Model}

As shown in Figure 6.1, we consider an uplink wireless energy harvesting sensor network with a set of $M$ sensor nodes, $\mathcal{N}=\{1,2, \ldots, M\}$. We consider a discrete-time model where time is divided into $N$ blocks. In each block, node $i \in \mathcal{N}$ has an event generated (sensed data) $\mathcal{E}_{i}[n]=1$ with probability $q_{i}$. Each node transmits its events to the base station either directly or through a cooperating node. Therefore, as shown in Figure 6.1, the network contains nodes of three categories; type $S_{1}$ nodes which transmit their events directly to the base station, type type $S_{2}$ nodes which transmit their events to the base station through a cooperating node, and cooperating nodes which transmit their events as well as they forward at least one type type $S_{2}$ node's events to the base station. We consider a real-time monitoring deployment scenario where events cannot be buffered for a later transmission [53]. We assume the nodes transmit over orthogonal frequency bands.

Each node is assumed to be equipped with energy harvesting devices and a rechargeable battery with capacity $K$. In the $n$-th time block, $n \in\{1,2, \ldots, N\}$, node $i \in \mathcal{N}$ harvests $E_{i}[n] \in\{0,1, \ldots, \tilde{K}\}$ amount of energy with corresponding probabilities $\left\{p_{i, 0}, p_{i, 1}, \ldots, p_{i, \tilde{K}}\right\}$, where $\tilde{K} \leqslant K$ is the maximum amount of energy that can be harvested in one block. We assume that the energy required for a reliable transmission per event from node $i$ to the base station is $\delta_{i}$, and between the nodes is $\tilde{\delta}$, where $\delta_{i}$ and $\tilde{\delta}$ are integers, and $\tilde{\delta}<\delta_{i}$ as the distance between the base station and any node is assumed to be larger than the distance between any two nodes.

Let $\mathcal{A}_{i} \in\{0,1\}$ be the transmission event of node $i \in \mathcal{N}$ with distribution $\operatorname{Pr}\left(\mathcal{A}_{i}=1\right)=$ $v_{i}$. In the $n$-th time block, if node $i$ has a transmission, i.e., $\mathcal{A}_{i}[n]=1$, the node is allowed to transmit only if it is energized, i.e., if its energy battery level $L_{i}[n] \geqslant \delta$, where $\delta=\delta_{i}$ if node 
$i$ is a type $S_{1}$ or a cooperating node and $\delta=\tilde{\delta}$ if node $i$ is a type $S_{2}$ node. At that time block, $\delta$ amount of energy will be depleted from its battery, and its transmission will be considered successfully delivered to the base station if node $i$ is a type $S_{1}$ node or a cooperating node, or to its cooperating node if node $i$ is a type $S_{2}$ node. We denote the successful transmission event of node $i \in \mathcal{N}$ by $\mathcal{B}_{i}$. Otherwise, i.e., if $L_{i}[n]<\delta$, the transmission will be discarded. Consequently, the energy battery level of the $(n+1)$-th time block of node $i$ is

$$
L_{i}[n+1]=\min \left\{L_{i}[n]+E_{i}[n]-\delta \mathbb{1}_{\left\{L_{i}[n] \geqslant \delta\right\}} \mathcal{A}_{i}[n], K\right\}
$$

where $\mathbb{1}_{\{\text {.\} }}$ is the indicator function. Because $L_{i}[n+1]$ depends only on the previous energy battery level $L_{i}[n]$, it is modeled as a Markov chain with state space $\{0,1, \ldots, K\}$, where the transition between any two states depends on $E_{i}[n]$ and $\mathcal{A}_{i}[n]$. Let $k$ and $j$ be the current and the previous states of the energy battery level, respectively, and let $z=k-j$ and $w=k-j+\delta$. Then given $v_{i}$ and $\boldsymbol{p}_{i}=\left[p_{i, 0}, p_{i, 1}, \ldots, p_{i, \tilde{K}}\right]^{T}$, the transition probability $Q_{j, k}^{(i)}=\operatorname{Pr}\left(L_{i}[n+1]=k \mid L_{i}[n]=j\right)$ of node $i \in \mathcal{N}$ between any two states $j, k \in\{0,1, \ldots, K\}$ is

$$
Q_{j, k}^{(i)}= \begin{cases}p_{i, z} & j<\delta, k<K, \\ p_{i, w} v_{i}+p_{i, z}\left(1-v_{i}\right) & j \geqslant \delta, k<K, \\ \sum_{\tilde{K}=z}^{\tilde{k}} p_{i, \tilde{k}} I_{1} & j<\delta, k=K, \\ \sum_{\tilde{k}=z}^{w-1} p_{i, \tilde{k}}\left(1-v_{i}\right) I_{2}+\sum_{\tilde{k}=w}^{\tilde{K}} p_{i, \tilde{k}} I_{3} & j \geqslant \delta, k=K,\end{cases}
$$

where $I_{1}=\mathbb{1}_{\{z \leqslant \tilde{K}\}}, I_{2}=\mathbb{1}_{\{z \leqslant w-1\}}$, and $I_{3}=\mathbb{1}_{\{w \leqslant \tilde{K}\}}$ are the indicator functions. Let $\mathbf{Q}^{(i)}$ be the transition matrix of the Markov chain whose $(j, k)$-th element is given by $Q_{j, k}^{(i)}$, and let $\pi_{i, 0}, \pi_{i, 1}, \ldots, \pi_{i, K}$ be its average (steady state) probabilities, where $\pi_{i, k}, k=\{0, \ldots, K\}$ is the average probability of the $k$-th energy battery level of node $i \in \mathcal{N}$. Then, the probability that node $i$ is energized is

$$
\triangle_{i}\left(v_{i}, \delta, \boldsymbol{p}_{i}\right)=\operatorname{Pr}\left(L_{i} \geqslant \delta\right)=\sum_{k=\delta}^{K} \pi_{i, k},
$$

and node $i$ has a successful transmission $\left(\mathcal{B}_{i}=1\right)$ is

$$
\tau_{i}\left(v_{i}, \delta, \boldsymbol{p}_{i}\right)=v_{i} \triangle_{i}\left(v_{i}, \delta, \boldsymbol{p}_{i}\right)=v_{i} \sum_{k=\delta}^{K} \pi_{i, k},
$$



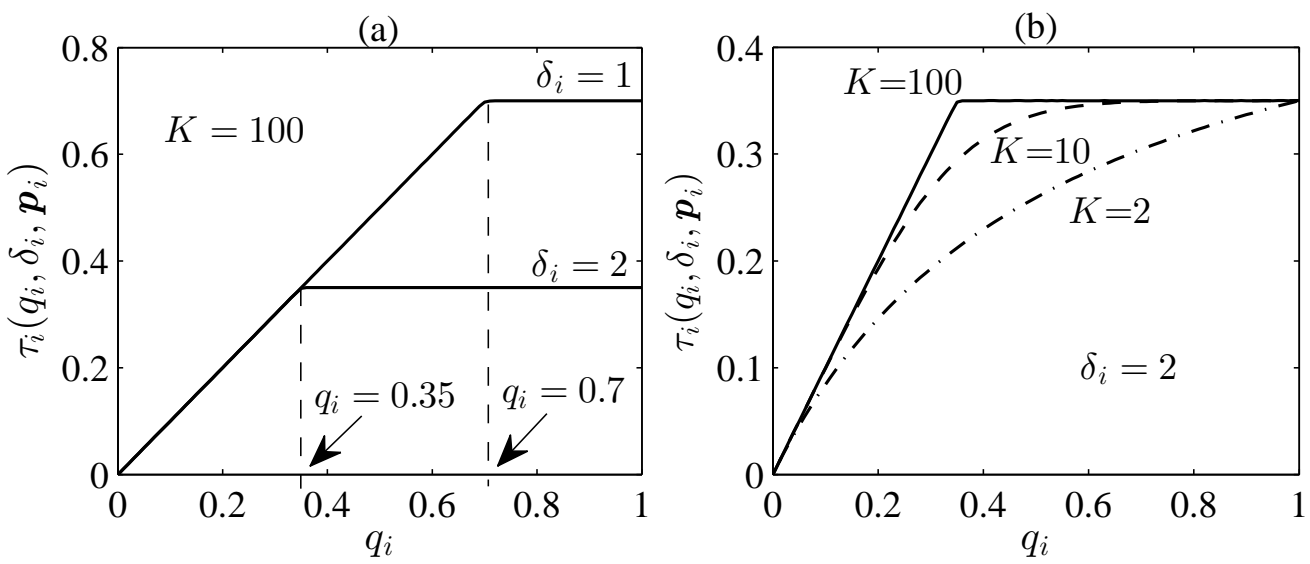

Figure 6.2: Successful transmission probability $\tau_{i}\left(q_{i}, \delta_{i}, \boldsymbol{p}_{i}\right)$ of node $i \in \mathcal{N}$ versus $q_{i}$ for different values of $\delta_{i}$ and $K$, and for $\boldsymbol{p}_{i}=\left[\begin{array}{ll}0.3 & 0.7\end{array}\right]$.

where $\pi_{i, 0}, \pi_{i, 1}, \ldots, \pi_{i, K}$ can be obtained using eigen decomposition of $\mathbf{Q}^{(i)} \forall K \geqslant \delta[65]$. In the special case when the battery capacity is much greater than $\delta$, i.e., $K \gg \delta$, by following the same derivation of $\sum_{k=\delta}^{K} \pi_{i, k}$ in [69] where a different problem is considered, we get

$$
\begin{aligned}
& \triangle_{i}\left(v_{i}, \delta, \boldsymbol{p}_{i}\right)= \begin{cases}1 & v_{i} \leqslant r_{i}, \\
\overline{\frac{E_{i}[n]}{v_{i} \delta}} & v_{i}>r_{i},\end{cases} \\
& \tau_{i}\left(v_{i}, \delta, \boldsymbol{p}_{i}\right)= \begin{cases}v_{i} & v_{i} \leqslant r_{i}, \\
\overline{\frac{E_{i}[n]}{\delta}} & v_{i}>r_{i},\end{cases}
\end{aligned}
$$

where $\overline{E_{i}[n]}$ is the average energy harvesting rate of node $i,\left(v_{i} \delta\right)$ is the energy depletion rate, and $r_{i}$ is the value of $v_{i}$ at which the energy harvesting rate of the node is equal to its energy depletion rate. $r_{i}$ can be expressed as

$$
r_{i}=\frac{\overline{E_{i}[n]}}{\delta} .
$$

\subsection{Successful transmission probability Characteriza- tion}

Let node $i \in \mathcal{N}$ be a type $S_{1}$ node, i.e., $\mathcal{A}_{i}=\mathcal{E}_{i}$ and $\delta=\delta_{i}$. Then, its successful transmission probability $\tau_{i}\left(q_{i}, \delta_{i}, \boldsymbol{p}_{i}\right)$ over $q_{i}$ for a given $\boldsymbol{p}_{i}$ and different values of $\delta_{i}$ and $K$ 
will be as shown in Figure 6.2. From the figure, we notice two key things.

First, we see in Figure 6.2 (a) that when $K$ is much greater than $\delta_{i}$, i.e., $K \gg \delta_{i}$, $\tau_{i}\left(q_{i}, \delta_{i}, \boldsymbol{p}_{i}\right)$ has two regions; linear which is the region over the range of $q_{i} \leqslant r_{i}$, and saturation which is the region over the range of $q_{i}>r_{i}$. When $q_{i}<r_{i}$, the energy harvesting rate of node $i$ is higher than its depletion rate. If operating in this region, the node will have all of its generated events successfully transmitted, i.e., $\tau_{i}\left(q_{i}, \delta_{i}, \boldsymbol{p}_{i}\right)=q_{i}$, and can operate as a cooperating node to successfully forward the fraction $d_{i}=r_{i}-q_{i}$ of events for another node. When $q_{i}>r_{i}$, the energy harvesting rate of the node is lower than its depletion rate. If operating in this region, the node will not have all of its generated events successfully transmitted, i.e, $\tau_{i}\left(q_{i}, \delta_{i}, \boldsymbol{p}_{i}\right)<q_{i}$, and can operate as a type $S_{2}$ node to improve its successful transmission probability. Note that, as $\delta_{i}$ decreases, the linear region increases at the expense of the saturation region, which implies that if $\tau_{i}\left(q_{i}, \delta_{i}, \boldsymbol{p}_{i}\right)=q_{i}$, then $\tau_{i}\left(q_{i}, \tilde{\delta}, \boldsymbol{p}_{i}\right)=q_{i}$ since $\tilde{\delta}<\delta_{i}$. We need this fact later to prove Proposition 1 .

Second, we see in Figure 6.2 (b) that when $K$ is close to $\delta_{i}$ and greater, i.e., $K \geqslant \delta_{i}$, $\tau_{i}\left(q_{i}, \delta_{i}, \boldsymbol{p}_{i}\right)$ has an additional region, called transient. The range of this region increases as $K$ gets closer to $\delta_{i}$, and it reaches the maximum size when $\delta_{i}=K$. As $K$ gets closer to $\delta_{i}$, more energy is wasted due to energy overflow, so that lower successful transmission probability is achieved. This wasted energy of node $i$ in this region can be exploited either by operating the node as a type $S_{2}$ where it transmits with a lower energy, or by operating the node as a cooperating node where it can successfully forward some events for another node.

In this chapter we consider the first case when $K \gg \delta_{i}$. The second case when $K \geqslant \delta_{i}$ can be considered as a future work.

\subsection{Problem Formulation}

The goal of this chapter is to maximize the total average of events that are successfully transmitted to the base station from the $M$ nodes over the $N$ time blocks, but without any node dropping below its base performance, defined as the performance obtained through direct transmission of its own events to the base station. As described in Section 6.2, each node in the network acts as either a type $S_{1}$, a type $S_{2}$, or a cooperating node. Thus, the 
set $\mathcal{N}$ can be partitioned into three subsets; $\mathcal{S}$ which includes all type $S_{1}$ nodes, $\mathcal{R}$ which includes all cooperating nodes, and $\mathcal{U}$ which includes all type $S_{2}$ nodes. Hence, $\mathcal{S}, \mathcal{R}, \mathcal{U} \subseteq \mathcal{N}$ and are disjoint.

Let $s \in \mathcal{S}$ be a type $S_{1}$ node, $r \in \mathcal{R}$ be a cooperating node, and $u$ be one of the type $S_{2}$ nodes that transmits through the cooperating node $r$, so that $u \in \mathcal{D}(r) \subseteq \mathcal{U}$. The type $S_{1}$ node $s$ has a transmission to the base station whenever it has an event generated, and this event is successfully delivered to the base station with probability $\tau_{s}\left(q_{s}, \delta_{s}, \boldsymbol{p}_{s}\right)$. This holds the same for the type $S_{2}$ node $u$. However, the type $S_{2}$ node $u$ transmits its event successfully to its cooperating node with probability $\tau_{u}\left(q_{u}, \tilde{\delta}, \boldsymbol{p}_{u}\right)$. As for the cooperating node $r$, it has a transmission to the base station not only whenever it has an event generated, but also whenever one of its type $S_{2}$ nodes $u \in \mathcal{D}(r)$ has a successful transmission to it. Thus, the cooperating node transmits its event or one of its type $S_{2}$ nodes' events successfully to the base station with probability $\tau_{r}\left(v_{r}, \delta_{r}, \boldsymbol{p}_{r}\right)$, where $v_{r}=\operatorname{Pr}\left(\mathcal{E}_{r}=1 \bigcup_{u \in \mathcal{D}(r)} \mathcal{B}_{u}=1\right)$ and $\operatorname{Pr}\left(\mathcal{B}_{u}=1\right)=\tau_{u}\left(q_{u}, \tilde{\delta}, \boldsymbol{p}_{u}\right)$. The cooperating node's event and its type $S_{2}$ nodes' events are independent and not mutually exclusive. Therefore, in the same time block, if more than one of them occurs and the cooperating node is energized, only one of them will be considered successfully transmitted, that will be the cooperating node's event if any, or the event of the type $S_{2}$ node $u \in \mathcal{D}(r)$ that achieves the smallest successful transmission probability to the base station. Because the base station receives only from type $S_{1}$ and cooperating nodes, the total number of the successful received events at the base station over the $N$ time blocks is

$$
\begin{gathered}
\mathcal{G}=\Gamma N, \\
\Gamma=\sum_{s \in \mathcal{S}} \tau_{s}\left(q_{s}, \delta_{s}, \boldsymbol{p}_{s}\right)+\sum_{r \in \mathcal{R}} \tau_{r}\left(v_{r}, \delta_{r}, \boldsymbol{p}_{r}\right),
\end{gathered}
$$

where $\Gamma$ is the data collection throughput of the network. In order to maximized (6.8), we maximize $\Gamma$ by optimizing the sets $\mathcal{S}, \mathcal{R}$, and $\mathcal{D}(r)$, and these sets can be optimized by optimally assigning each node in $\mathcal{N}$ as either a type $S_{1}$ node, a cooperating node, or a type $S_{2}$ node of which cooperating node. A node $i \in \mathcal{N}$ is optimally assigned to be a cooperating node of some type $S_{2}$ nodes if, 1) the total number of events that are successfully transmitted from that cooperating node to the base station, including its type $S_{2}$ nodes' events, is higher 
than or equal to the total number of events that are successfully transmitted from that cooperating node and its type $S_{2}$ nodes when each one of them transmits its events on its own to the base station, and 2) that relay can forward more events for each one of its type $S_{2}$ nodes than any other cooperating node in the network. If node $i$ cannot be a cooperating node or a type $S_{2}$ node, then it will be a type $S_{1}$ node. Consequently, the optimization problem can be formulated as

$$
\begin{array}{ll}
\max _{\mathcal{S}, \mathcal{R}, \mathcal{D}(r)} & \Gamma \\
\text { s.t } & \tau_{r}\left(v_{r}, \delta_{r}, \boldsymbol{p}_{r}\right) \geqslant \tau_{r}\left(q_{r}, \delta_{r}, \boldsymbol{p}_{r}\right)+\sum_{u \in \mathcal{D}(r)} \tau_{u}\left(q_{u}, \delta_{u}, \boldsymbol{p}_{u}\right), \quad \forall r \in \mathcal{R} \\
& \left.\tau_{r}\left(v_{r}, \delta_{r}, \boldsymbol{p}_{r}\right)\right|_{u \in \mathcal{D}(r)}-\left.\tau_{r}\left(v_{r}, \delta_{r}, \boldsymbol{p}_{r}\right)\right|_{\mathcal{D}(r) \backslash\{u\}} \geqslant \\
& \left.\tau_{j}\left(v_{j}, \delta_{j}, \boldsymbol{p}_{j}\right)\right|_{u \in \mathcal{D}(j)}-\left.\tau_{j}\left(v_{j}, \delta_{j}, \boldsymbol{p}_{j}\right)\right|_{\mathcal{D}(j) \backslash\{u\}}, \quad \forall r, j \in \mathcal{R} \\
& |\mathcal{S}|+|\mathcal{R}|+\sum_{r \in \mathcal{R}}|\mathcal{D}(r)|=M .
\end{array}
$$

\subsection{A brute force approach}

In (6.10), the optimal sets $\mathcal{S}^{\star}, \mathcal{R}^{\star}$, and $\mathcal{D}^{\star}(r)$ can be found by examining all the possible combinations of the nodes in set $\mathcal{N}$ as a type $S_{1}$, a cooperating node, or a type $S_{2}$. To reduce the computation complexity, some of these combinations can be excluded as they do not apply to the system model. In the system model, we have that a cooperating node serves at least one type $S_{2}$ node, and a type $S_{2}$ node uses only one cooperating node. This implies that the number of type $S_{2}$ nodes in the network must be equal to or larger than the number of cooperating nodes. i.e., if the network has $|\mathcal{S}|$ type $S_{1}$ nodes, $0 \leqslant|\mathcal{S}| \leqslant M$, then the number of cooperating nodes has to be $|\mathcal{R}| \leqslant\lfloor(M-|\mathcal{S}|) / 2\rfloor$ and the number of type $S_{2}$ nodes $|\mathcal{U}|=M-|\mathcal{S}|-|\mathcal{R}|$. Also, this implies that the number of type $S_{1}$ nodes cannot be $M-1$. This is because if the remaining node is categorized as a cooperating node, it will not be associated with a type $S_{2}$ node and vice versa. Using these constraints, the number of all possible combinations as a function of $M$ is

$$
\mathcal{C}=\left[\sum_{|\mathcal{S}|=1}^{M-2} \sum_{|\mathcal{R}|=1}^{\lfloor(M-|\mathcal{S}|) / 2\rfloor}\left(\begin{array}{c}
|\mathcal{R}|+|\mathcal{U}| \\
|\mathcal{R}|
\end{array}\right)\left(\begin{array}{c}
M \\
|\mathcal{S}|
\end{array}\right)+\sum_{|\mathcal{R}|=1}^{\lfloor M / 2\rfloor}\left(\begin{array}{c}
M \\
|\mathcal{R}|
\end{array}\right)\right] \mathcal{Q}+1
$$




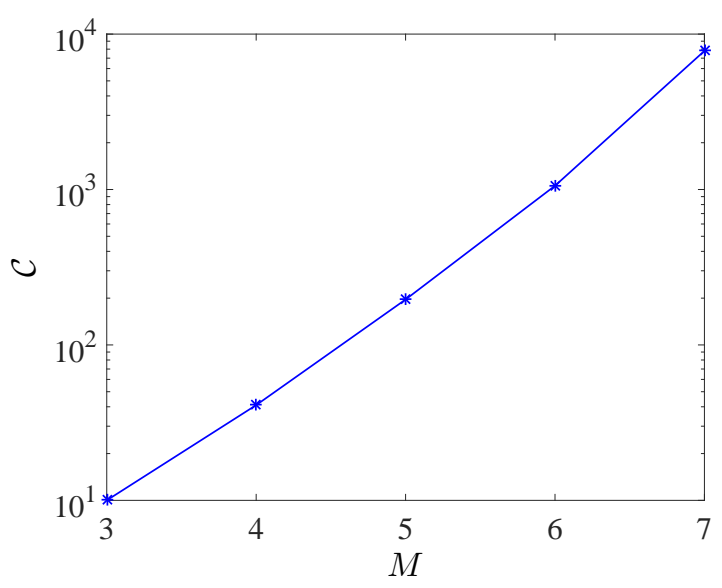

Figure 6.3: The number of all possible combinations increases exponentially with $M$.

$$
\mathcal{Q}=\left(1 \mathbb{1}_{(|\mathcal{R}|=1)}+|\mathcal{R}| ! \mathbb{1}_{(|\mathcal{R}|=|\mathcal{U}| \neq 1)}+\left(|\mathcal{R}|^{|\mathcal{U}|}-|\mathcal{R}|\right) \mathbb{1}_{(1<|\mathcal{R}|<|\mathcal{U}|)}\right)
$$

where the first term in (6.11) calculates all the possible combinations when the network has $|\mathcal{S}|$ type $S_{1}$ nodes, $0<|\mathcal{S}| \leqslant M-2$, the second term calculates all the possible combinations when the network has only cooperating nodes and type $S_{2}$ nodes, i.e., $|\mathcal{S}|=0$, and the added 1 is for the only combination when all the nodes are type $S_{1}$ nodes, i.e., $|\mathcal{S}|=M$. Although the number of the examined combinations is reduced, it is still relatively high. As shown in Figure 6.3, it scales exponentially with $M$, meaning that the computation complexity of the brute force approach will also scale exponentially. Therefore, we next propose algorithms that finds the optimal $\Gamma$ with less computation complexity.

\subsection{Centralized Algorithm}

Algorithm 6.1 illustrates the centralized algorithm that finds the optimal sets $\mathcal{S}^{\star}, \mathcal{R}^{\star}$, and $\mathcal{D}^{\star}(r)$ that maximizes $\Gamma$ in (6.10). For a given set of nodes $\mathcal{N}$, the algorithm initializes two sets $\mathcal{Y}$ and $\mathcal{Z}$, where $\mathcal{Y}, \mathcal{Z} \subseteq \mathcal{N}$ and $\mathcal{Z}=\mathcal{Y}^{\mathrm{c}}$. Set $\mathcal{Y}$ contains every node in $\mathcal{N}$ that is a candidate to be a cooperating node while set $\mathcal{Z}$ contains every node in $\mathcal{N}$ that is a candidate to be a type $S_{2}$ node. A node $i \in \mathcal{N}$ is said to be a cooperating node candidate if its energy harvesting rate is higher than or equal to its energy depletion rate, i.e., $q_{i} \leqslant r_{i}$. Otherwise, the node is said to be a type $S_{2}$ candidate. Note that when the node has its energy harvesting rate equal to its energy depletion rate, i.e., $q_{i}=r_{i}$, it is still considered 
a cooperating node candidate in order to make the algorithm less complex. The algorithm also initializes the sets $\mathcal{R}=\{\emptyset\}$ and $\mathcal{D}(i)=\{\emptyset\} \forall i \in \mathcal{Y}$.

Proposition 1 : If all the nodes are cooperating node candidates, i.e., $q_{i} \leqslant r_{i} \forall i \in \mathcal{N}$, $|\mathcal{Y}|=M$, and $|\mathcal{Z}|=0$, then the maximum throughput of the network is achieved when all the nodes are type $S_{1}$ nodes, i.e., $\mathcal{S}^{\star}=\mathcal{N}, \mathcal{R}^{\star}=\mathcal{D}^{\star}(i)=\{\emptyset\} \forall i \in \mathcal{Y}$.

Proof : We have seen in Section 6.3 that $\tau_{i}\left(q_{i}, \delta_{i}, \boldsymbol{p}_{i}\right)=q_{i}$ for node $i \in \mathcal{N}$ when $q_{i} \leqslant r_{i}$, and $\tau_{i}\left(q_{i}, \tilde{\delta}, \boldsymbol{p}_{i}\right)=q_{i}$ if $\tau_{i}\left(q_{i}, \delta_{i}, \boldsymbol{p}_{i}\right)=q_{i}$. Given this and assuming that node $i$ is a cooperating node of $|\mathcal{D}(i)|$ type $S_{2}$ nodes, the first constraint in (6.10) becomes

$$
v_{i} \triangle_{i}\left(v_{i}, \delta_{i}, \boldsymbol{p}_{i}\right) \geqslant q_{i}+\sum_{u \in \mathcal{D}(i)} q_{u}
$$

Here $v_{i}=\operatorname{Pr}\left(\mathcal{E}_{i}=1 \bigcup_{u \in \mathcal{D}(i)} \mathcal{E}_{u}=1\right)$. Because the events of the cooperating node and type $S_{2}$ nodes are independent and not mutually exclusive, the left-hand-side of (6.13) will be less than its right-hand-side $\forall \triangle_{i}\left(v_{i}, \delta_{i}, \boldsymbol{p}_{i}\right) \leqslant 1$. Thus, the constraint in (6.13) will be met only if $\mathcal{D}(i)=\{\emptyset\}$, where in this case both sides of (6.13) will equal to $q_{i}$, i.e., node $i \in \mathcal{N}$ will transmit only its events, acting as a type $S_{1}$. When all the nodes are type $S_{1}$, the third constraint in (6.10) will be met, and the second constraint will also be met where both of its sides will be 0 .

Proposition 2: If all the nodes are type $S_{2}$ candidates, i.e., $q_{i}>r_{i} \forall i \in \mathcal{N},|\mathcal{Y}|=0$, and $|\mathcal{Z}|=M$, then the maximum throughput is achieved when all the nodes are type $S_{1}$ nodes, i.e., $\mathcal{S}^{\star}=\mathcal{N}, \mathcal{R}^{\star}=\mathcal{D}^{\star}(i)=\{\emptyset\} \forall i \in \mathcal{Y}$.

Proof : We showed in Section 6.3 that $\tau_{i}\left(q_{i}, \delta_{i}, \boldsymbol{p}_{i}\right)$ of node $i \in \mathcal{N}$ remains constant $\forall q_{i}>r_{i}$. Let node $i$ be a cooperating node of $|\mathcal{D}(i)|$ type $S_{2}$ nodes. Because $v_{i}=\operatorname{Pr}\left(\mathcal{E}_{i}=\right.$ $\left.1 \bigcup_{u \in \mathcal{D}(i)} \mathcal{B}_{u}=1\right)>q_{i}>r_{i}$, then $\tau_{i}\left(v_{i}, \delta_{i}, \boldsymbol{p}_{i}\right)=\tau_{i}\left(q_{i}, \delta_{i}, \boldsymbol{p}_{i}\right) \forall i \in \mathcal{N}$. Thus, the first constraint in (6.10) becomes

$$
\tau_{i}\left(q_{i}, \delta_{i}, \boldsymbol{p}_{i}\right) \geqslant \tau_{i}\left(q_{i}, \delta_{i}, \boldsymbol{p}_{i}\right)+\sum_{u \in \mathcal{D}(i)} \tau_{u}\left(q_{u}, \delta_{u}, \boldsymbol{p}_{u}\right)
$$

and it will be met only if $\mathcal{D}(i)=\{\emptyset\}$, i.e., node $i$ transmits only its events, acting as a type $S_{1}$.

If the network has some nodes that are cooperating node candidates and some nodes that are type $S_{2}$ candidates, i.e., $|\mathcal{Y}| \neq 0$ and $|\mathcal{Y}| \neq M$, the nodes in $\mathcal{Y}$ will be ordered decreasingly 


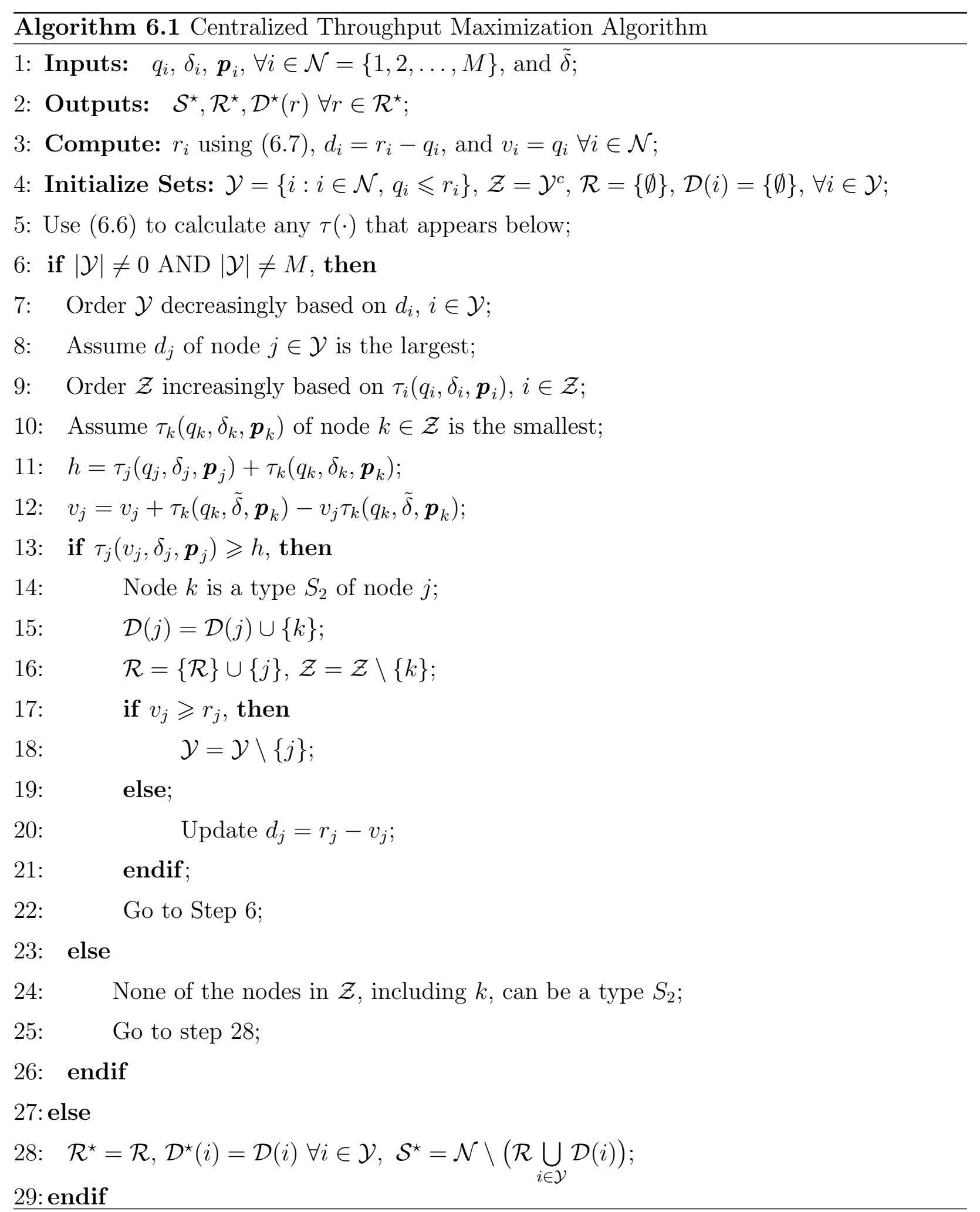


based on their fractions of events that they can forward for another node $(d)$. On the other hand, the nodes in $\mathcal{Z}$ will be ordered increasingly based on their successful transmission probabilities to the base station. Ordering these sets will ensure that if the first constraint in (6.10) is met, then the second constraint will also be met. Assume that $d_{j}$ of node $j \in \mathcal{Y}$ is the largest and $\tau_{k}\left(q_{k}, \delta_{k}, \boldsymbol{p}_{k}\right)$ of node $k \in \mathcal{Z}$ is the smallest. Then, node $k$ will be tested to be a type $S_{2}$ of node $j$ or not by checking the first constraint. If the first constraint is met, then node $k$ will be a type $S_{2}$ of node $j$. After that, node $k$ will be removed from set $\mathcal{Z}$ and added to set $\mathcal{D}(j)$, and node $j$ will be added to the set of cooperating nodes $\mathcal{R}$. After adding node $k$ to its set, node $j$ will be removed from set $\mathcal{Y}$ only if its energy harvesting rate becomes lower than or equal to its energy depletion rate, i.e., $v_{j} \geqslant r_{j}$. Otherwise, it will remain in the set and its $d_{j}$ will be updated. After updating and reordering the two sets $\mathcal{Y}$ and $\mathcal{Z}$, the same procedure will continue with the next nodes in $\mathcal{Y}$ and $\mathcal{Z}$. If the first constraint is not met, however, node $k$ cannot be a type $S_{2}$ of node $j$, or any of the remaining cooperating node candidates as they have a lower fraction of events that they can forward. This holds the same for the remaining type $S_{2}$ candidates as they have higher successful transmission probabilities than node $k$. And thus the test will terminate.

Note that if two nodes or more in $\mathcal{Y}$ or $\mathcal{Z}$ have the same fraction of events that they can forward or have the same successful transmission probability, respectively, the algorithm will arbitrarily order them. After the test ends or terminates, the sets $\mathcal{R}, \mathcal{D}(i) \forall i \in \mathcal{Y}$, and $\mathcal{S}$ will be optimized. Using these sets, the optimal $\Gamma$ can be calculated from (6.9). As stated in Section 6.1, the algorithm is run at the base station which broadcastes a message that has the assignment of each node after the test ends. If a node is assigned to be a type $S_{1}$ or a cooperating node, then its transmission path is directly to the base station. If it is assigned to be a type $S_{2}$ of some cooperating node, then its transmission path to the base station is through that cooperating node.

There is no close form expression for the number of iterations required to find the optimal solution because it depends on the system parameters. However, we can provide an upperbound expression, which can be derived from the worst scenario, that is when the network has only one cooperating node candidate and the rest of the nodes are type $S_{2}$ candidates, and these type $S_{2}$ candidates are assigned to be type $S_{2}$ nodes of that cooperating node 
candidate after running the algorithm. In this scenario, the number of iterations will be the same as the number of type $S_{2}$ candidates, which is $M-1$. Thus, the algorithm's complexity is $\mathcal{O}(M)$, which is linear.

\subsection{Distributed Algorithm}

Algorithms 6.2 and 6.3 summarize the distributed algorithm that finds the optimal solution for (6.10). Algorithm 6.2 is for node $i \in \mathcal{N}$ if it is a type $S_{2}$ candidate, and Algorithm 6.3 is for node $i \in \mathcal{N}$ if it is a cooperating node candidate. Initially, each node $i \in \mathcal{N}$ calculates its $r_{i}$ using (6.7) when $\delta=\delta_{i}$, where by comparing $q_{i}$ with $r_{i}$, node $i$ can know if it is a type $S_{2}$ candidate or a cooperating node candidate. If node $i$ is a type $S_{2}$ candidate, then as illustrated in Algorithm 6.2, the node first calculates its $\tau_{i}\left(q_{i}, \tilde{\delta}, \boldsymbol{p}_{i}\right)$ and $\tau_{i}\left(q_{i}, \delta_{i}, \boldsymbol{p}_{i}\right)$ using (6.6). And then at the beginning of the time block, the node starts a timer of a duration $t_{i}=\tau_{i}\left(q_{i}, \delta_{i}, \boldsymbol{p}_{i}\right)$ time units. The timer is to give priority for the type $S_{2}$ candidate that achieves the smallest successful transmission probability to the base station to be assisted first, which is similar to the centralized algorithm where the type $S_{2}$ candidates are given priorities by ordering them increasingly based on their successful transmission probabilities. If none of the other type $S_{2}$ candidates has broadcasted its successful transmission probabilities before the time $t_{i}$ ends, which happens only if node $i$ has the shortest timer length, node $i$ will broadcast its $\tau_{i}\left(q_{i}, \tilde{\delta}, \boldsymbol{p}_{i}\right)$ and $\tau_{i}\left(q_{i}, \delta_{i}, \boldsymbol{p}_{i}\right)$ to the network right after its timer expires, and only the cooperating node candidates will receive them. After that, node $i$ will wait for an ACK from a cooperating node candidate. If the cooperating node candidate $j \in \mathcal{N}$ can be a cooperating node of node $i$, then it will broadcast an ACK to inform the type $S_{2}$ candidates that node $i$ became its type $S_{2}$ node. In this case, node $i$ will be a type $S_{2}$ of node $j$ and it will terminate the test. If no ACK is received, i.e., none of the cooperating node candidates can be its cooperating node, then node $i$ will be a type $S_{1}$. If one of the other type $S_{2}$ candidates has broadcasted its successful transmission probabilities before the time $t_{i}$ ends, node $i$ will turn off its timer and wait for an ACK from a cooperating node candidate $j \in \mathcal{N}$. If an ACK is received, then the node will wait for the next time block to start the procedure all over again. If not, node $i$ will operate as a type $S_{1}$. 


\section{Algorithm 6.2 Distributed Algorithm for a type $S_{2}$ Candidate Node $i \in \mathcal{N}$}

1: Inputs: $q_{i}, \delta_{i}, \tilde{\delta}, \boldsymbol{p}_{i}$;

2: Outputs: Category of node $i$;

3: Compute: $\tau_{i}\left(q_{i}, \tilde{\delta}, \boldsymbol{p}_{i}\right)$ and $\tau_{i}\left(q_{i}, \delta_{i}, \boldsymbol{p}_{i}\right)$ using (6.6);

4: At the beginning of a time block,

5: Set and start a timer $t_{i}=\tau_{i}\left(q_{i}, \delta_{i}, \boldsymbol{p}_{i}\right)$;

6: if none of the other type $S_{2}$ candidates broadcasted its $\tau(\cdot) \mathrm{s}$, then

7: When the timer expires,

8: $\quad$ Broadcast $\tau_{i}\left(q_{i}, \tilde{\delta}, \boldsymbol{p}_{i}\right)$ and $\tau_{i}\left(q_{i}, \delta_{i}, \boldsymbol{p}_{i}\right)$;

9: $\quad$ Wait for an ACK from a cooperating node candidate $j \in \mathcal{N}$;

10: if an ACK received before the end of the time block, then

11: $\quad$ Node $i$ is a type $S_{2}$ of the cooperating node candidate $j$;

12: $\quad$ else

13: $\quad$ Node $i$ is a type $S_{1}$;

14: $\quad$ endif

15: else

16: $\quad$ Turn off your timer when $\tau(\cdot)$ s are started to be broadcasted;

17: $\quad$ Wait for an ACK from a cooperating node candidate $j \in \mathcal{N}$;

18: if an ACK received before the end of the time block, then

19: $\quad$ Go to step 4;

20: $\quad$ else

21: $\quad$ Node $i$ is a type $S_{1}$;

22: $\quad$ endif 


\section{$\overline{\text { Algorithm 6.3 Distributed Algorithm for a Cooperating Node Candidate } i \in \mathcal{N}}$}

1: Inputs: $q_{i}, \delta_{i}, \boldsymbol{p}_{i}$;

2: Outputs: Category of node $i$;

3: Compute: $r_{i}$ using (6.7), and $d_{i}=r_{i}-q_{i}$, and set $v_{i}=q_{i}$;

4: At the beginning of a time block,

5: Wait for a transmitted $\tau(\cdot)$ s from a type $S_{2}$ candidate $j \in \mathcal{N}$;

6: if any $\tau(\cdot)$ s received before the end of the time block, then

7: Check the first constraint in (6.10) including any previous type $S_{2}$ nodes;

8: if the constraint is met, then

9: $\quad$ Set and start a timer $t_{i}=\left(1-d_{i}\right)$;

10: if none of the other cooperating node candidates broadcasted ACK, then

11: When the timer expires,

12: $\quad$ Broadcast an ACK that node $i$ is a cooperating node of node $j$;

13: $\quad$ Calculate $v_{i}=v_{i}+\tau_{j}\left(q_{j}, \tilde{\delta}, \boldsymbol{p}_{j}\right)-v_{i} \tau_{j}\left(q_{j}, \tilde{\delta}, \boldsymbol{p}_{j}\right)$;

14: $\quad$ if $v_{i}<r_{i}$, then

15: $\quad$ Update $d_{i}=r_{i}-v_{i}$, go to step 4;

16: else

17: $\quad$ Go to step 31;

18: endif

19: else

20: $\quad$ Turn off your timer, go to step 4;

21: endif

22: else

23: if the constraint has been met before this time block, then

24: $\quad$ Node $i$ is a cooperating node for the nodes that have met the constraint;

25: $\quad$ else

26: $\quad$ Node $i$ is a type $S_{1}$;

27: $\quad$ endif

28: endif

29: else

30: Go to step 23;

31: endif 
If node $i$ is a cooperating node candidate, then as illustrated in Algorithm 6.3, the node first calculates its $d_{i}=r_{i}-q_{i}$ and sets $v_{i}=q_{i}$. At the beginning of a time block, node $i$ waits for any broadcasted successful transmission probabilities $\tau(\cdot)$ s from a type $S_{2}$ candidate $j \in \mathcal{N}$. If any $\tau(\cdot)$ s are received, node $i$ will first check the first constraint in (6.10). And if the constraint including any previous type $S_{2}$ nodes is met, the node will start a timer of a duration $t_{i}=\left(1-d_{i}\right)$ time units, where the node of the largest fraction of the extra events that it can forward will assist first. If none of the other cooperating node candidates broadcasted an ACK before the time $t_{i}$ ends, then node $i$ will broadcast an ACK after the time $t_{i}$ ends to inform all the type $S_{2}$ candidates that node $j$ became its type $S_{2}$ node. And then node $i$ will update its $v_{i}$ and check if its energy harvesting rate is still higher than its energy depletion rate or not. If yes, then it will update its $d_{i}$, and then wait for the next time block to start the procedure all over again as it may still be able to assist with the transmissions of another type $S_{2}$ candidate. Otherwise, it will terminate the test. If any of the other cooperating node candidates broadcasted an ACK before $t_{i}$ ends, then node $i$ will turn off its timer and wait for the next time block to start again. On the other hand, at the beginning of a time block, if any $\tau(\cdot)$ s are received and the first constraint is not met, or if no $\tau(\cdot)$ s are received, then node $i$ will be a cooperating node if the constraint has been met with any type $S_{2}$ candidates before the current time block. Otherwise, it will be a type $S_{1}$.

In the distributed algorithm, the optimal solution is found in the first time block in which there is no ACK broadcasted. By that time block, each node will already be optimally assigned a role. Note that, if two or more type $S_{2}$ candidates or if two or more cooperating node candidates have the same timer length, the priority can be given for the node of the largest ID number. That is, when the type $S_{2}$ candidates of the same timer length broadcast their $\tau(\cdot)$ s, the cooperating node candidates of the same timer length each will broadcast an ACK back that has the the largest ID among these type $S_{2}$ candidates if the first constraint is met. In turn, the type $S_{2}$ candidate of the largest ID number will chose the cooperating node candidate of the largest ID number as a cooperating node, and then broadcast an ACK to inform the cooperating node candidates that the cooperating node candidate of the largest ID number has been chosen to be its cooperating node, and so on.

In the distributed algorithm, the number of iterations required to find the optimal solution 
has the same upper-bound as the centralized algorithm. Therefore, the complexity of the distributed algorithm is also linear. Here, the upper-bound of the number of iterations is calculated from Algorithm 6.3 as it is more complex than Algorithm 6.2, and from the same worst scenario discussed in the previous section. Although one of them could save the nodes more energy than the other, both the centralized and distributed algorithms achieve the same throughput over the long term, i.e., when $N \rightarrow \infty$. This is because when $N \rightarrow \infty$, the average probability, given by (6.3), that a node is energized over the $N$ time blocks will be the same regardless of the remaining energy after finding the optimal solution, which is due to the fact that the energy battery level of a node is modeled as a Markov chain. Hence, each node will achieve the same successful transmission probability in (6.4), which will lead to the same network throughput.

\subsection{Variable Transmit Power}

The centralized and distributed algorithms designed earlier can be adapted for the scenario when the transmit power between the nodes is variable. Here, the transmit power between any two nodes $i, j \in \mathcal{N}$ will be $\delta_{i, j}$ instead of $\tilde{\delta}$. Thus, the successful transmission probability from a type $S_{2}$ node $u$ to its cooperating node $r$ will be $\tau_{u}\left(q_{u}, \delta_{u, r}, \boldsymbol{p}_{u}\right)$, which still can be calculated using (6.6).

When the transmit power between the nodes is variable, we cannot guarantee that if the first constraint in (6.10) is met, then the second constraint will also be met. Hence, each type $S_{2}$ candidate in $\mathcal{Z}$ has to be tested as a type $S_{2}$ of each cooperating node candidate in $\mathcal{Y}$, which implies that set $\mathcal{Y}$ does not need to be ordered. For this scenario, therefore, we can modify the centralized algorithm illustrated in Algorithm 6.1 as follows. After creating the two sets $\mathcal{Z}$ and $\mathcal{Y}$, we first order set $\mathcal{Z}$ increasingly. Then, we find the cooperating node candidates that meet the first constraint with the first type $S_{2}$ candidate in $\mathcal{Z}$, and the one that meets the second constraint among these cooperating node candidates will be a cooperating node of that type $S_{2}$ candidate. After that, we remove that type $S_{2}$ candidate from set $\mathcal{Z}$, and then update the two sets $\mathcal{Z}$ and $\mathcal{Y}$. After updating these two sets, we repeat the same steps for the next type $S_{2}$ candidate in $\mathcal{Z}$. 
As for the distributed algorithm, a type $S_{2}$ candidate will broadcast its $\overline{E[n]}$ and $q$ instead of its $\tau(\cdot) \mathrm{s}$ as in the fixed transmit power scenario. Hence, the $\tau(\cdot) \mathrm{s}$ will be calculated at the cooperating node candidates in order to reduce the computation complexity. This, however, requires the assumption that each node knows the transmit power between it and any other node in the network. Based on these modifications, Algorithm 6.3 can be changed for the variable transmit power scenario as follows. In each time block, a cooperating node candidate first calculates the $\tau(\cdot)$ s of the type $S_{2}$ candidate of the shortest timer length, and then checks the first and the second constraints. After that, it broadcasts an ACK, which will have the number of events that it can forward to that type $S_{2}$ candidate without setting any timer. In turn, that type $S_{2}$ candidate will select the cooperating node candidate that can forward the largest number of events to it by its ID, and broadcast this in an ACK to the cooperating node candidates. Selecting the cooperating node candidate that can forward the largest number of events as well as broadcasting $\overline{E[n]}$ and $q$ instead of $\tau(\cdot)$ s will be the only change in Algorithm 6.2.

In the adaptive transmit power algorithms, the upper bound of the number of iterations that is required to find the optimal solution is calculated from the worst scenario, that is when $M$ is even, and the half of the nodes are cooperating node candidates and the other half are type $S_{2}$ candidates, and each cooperating node candidate still can serve as a cooperating node candidate after it adds a type $S_{2}$ to its set. In this scenario, because each type $S_{2}$ candidate will be tested as a type $S_{2}$ of each cooperating node candidate, the total number of iterations will be $\left(\frac{M}{2}\right)^{2}$. Thus, the algorithms' complexity is $\mathcal{O}\left(M^{2}\right)$, which is quadratic.

\subsection{Simulation Results}

Although the algorithms were designed for an arbitrary number of nodes $M$, for simplicity, in the simulations we consider a network of three nodes, i.e., $\mathcal{N}=\{1,2,3\}$. We use three different sets of parameters. The first set of parameters is $q_{1}=q_{2}=q_{3}=0.2, \boldsymbol{p}_{1}=\boldsymbol{p}_{2}=[0.2$ 0.8], $\boldsymbol{p}_{3}=\left[\begin{array}{ll}0.8 & 0.2\end{array}\right], \tilde{K}=1, K=100, N=10^{5}, \delta_{1}=\delta_{2}=\delta_{3}=2, \tilde{\delta}=1$. The second set of parameters is $q_{1}=q_{2}=q_{3}=0.2, \boldsymbol{p}_{1}=\boldsymbol{p}_{2}=\boldsymbol{p}_{3}=\left[\begin{array}{ll}0.2 & 0.8\end{array}\right], \tilde{K}=1, K=100, N=10^{5}$, $\delta_{1}=\delta_{2}=\delta_{3}=2, \tilde{\delta}=1$. The third set of parameters is $q_{1}=0.2, q_{2}=0.8, q_{3}=0.2$, 
Table 6.1: All Possible Scenarios of the Network

\begin{tabular}{cccc}
\hline \hline Scenario & Node 1 & Node 2 & Node 3 \\
\hline 1 & type $S_{1}$ & type $S_{1}$ & type $S_{1}$ \\
2 & type $S_{1}$ & type $S_{2}$ & Cooperating node \\
3 & type $S_{1}$ & Cooperating node & type $S_{2}$ \\
4 & type $S_{2}$ & type $S_{1}$ & Cooperating node \\
5 & Cooperating node & type $S_{1}$ & type $S_{2}$ \\
6 & type $S_{2}$ & Cooperating node & type $S_{1}$ \\
7 & Cooperating node & type $S_{2}$ & type $S_{1}$ \\
8 & Cooperating node & type $S_{2}$ & type $S_{1}$ \\
9 & type $S_{1}$ & Cooperating node & type $S_{2}$ \\
10 & type $S_{2}$ & type $S_{2}$ & Cooperating node \\
\hline
\end{tabular}

$\boldsymbol{p}_{1}=\left[\begin{array}{llllll}0.025 & 0.025 & 0.025 & 0.025 & 0.1 & 0.8\end{array}\right], \boldsymbol{p}_{2}=\left[\begin{array}{llllll}0.2 & 0.3 & 0.3 & 0.2 & 0 & 0\end{array}\right], \boldsymbol{p}_{3}=\left[\begin{array}{llllll}0.1 & 0.1 & 0.2 & 0.3 & 0.3\end{array}\right.$ $0], \tilde{K}=5, K=100, N=10^{5}, \delta_{1}=\delta_{2}=\delta_{3}=6, \delta_{1,2}=2, \delta_{1,3}=4, \delta_{2,3}=1$. Each set is contained in its figure's caption.

We simulate both the fixed and variable transmit power scenarios. In these two scenarios, the optimal $\Gamma$ is calculated using the proposed algorithms and brute force approach. In the brute force approach, using the constraints mentioned in Section 6.5, Table 6.1 shows all the possible scenarios or combinations of the nodes' assignments that were examined. In the same row/scenario of Table 6.1 when there is a type $S_{2}$ node and a cooperating node, it means that the type $S_{2}$ node is a type $S_{2}$ node of that cooperating node. For each scenario, $\Gamma$ is calculated by simulation using Monte Carlo technique, and analytically using (6.9). In the centralized algorithm, the nodes in $\mathcal{Y}$ or $\mathcal{Z}$ that have the same fraction of events that they can forward or have the same successful transmission probability, respectively, are ordered arbitrarily using a uniform distribution. All the simulations are done using Matlab. We compare our approach with the cooperative transmission scenario discussed in Chapter 2, where each pair of nodes in the network form a three-relay channel with the base station. 

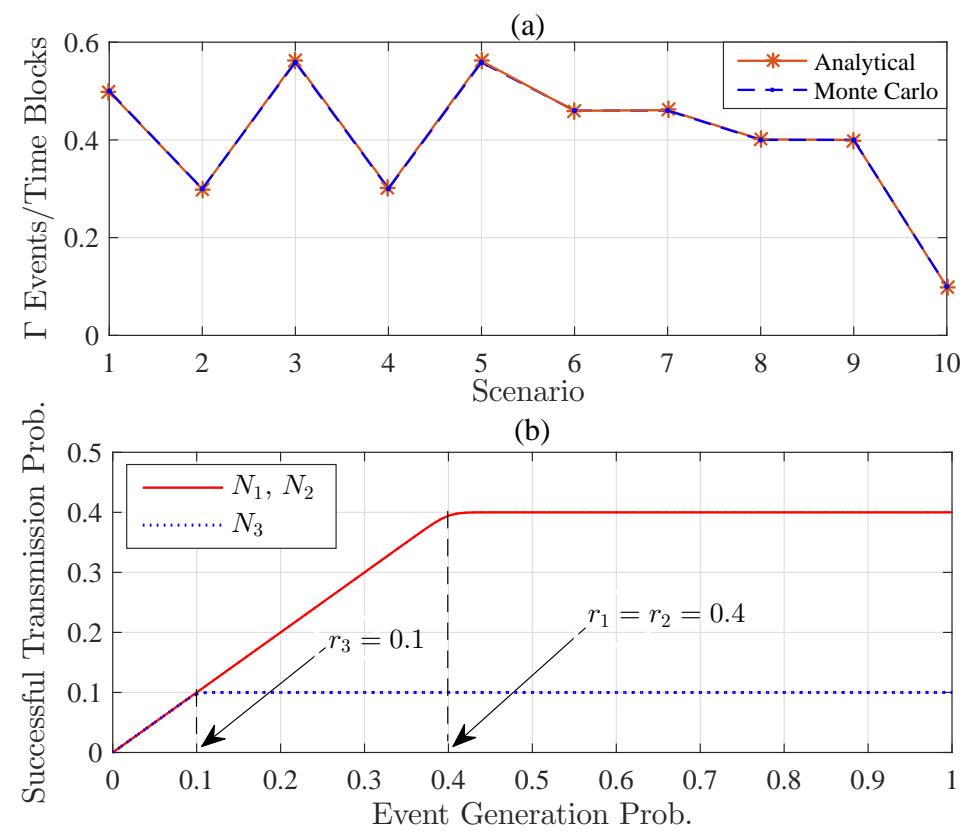

Figure 6.4: Figure (a) shows the network throughput for all the scenarios shown in Table 6.1. Figure (b) shows the successful transmission probability of $N_{1}, N_{2}$, and $N_{3}$. Parameters used: $q_{1}=q_{2}=q_{3}=0.2, \boldsymbol{p}_{1}=\boldsymbol{p}_{2}=\left[\begin{array}{ll}0.2 & 0.8\end{array}\right], \boldsymbol{p}_{3}=\left[\begin{array}{ll}0.8 & 0.2\end{array}\right], \tilde{K}=1, K=100, N=10^{5}$, $\delta_{1}=\delta_{2}=\delta_{3}=2, \tilde{\delta}=1$.

(a)

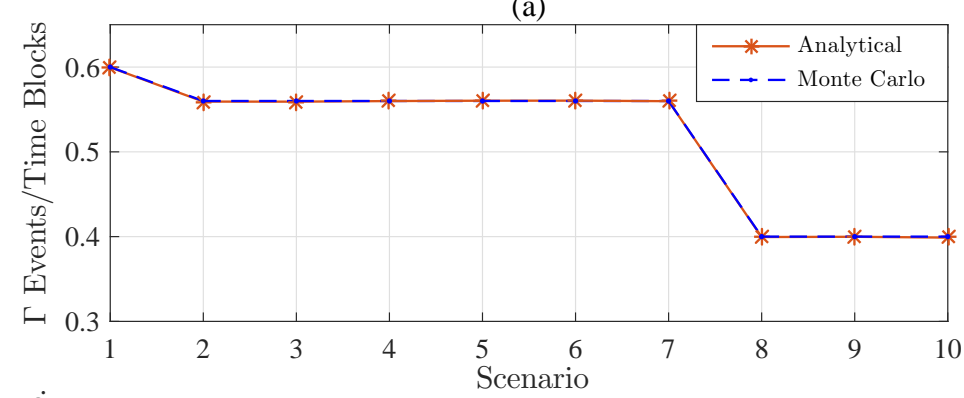

(b)

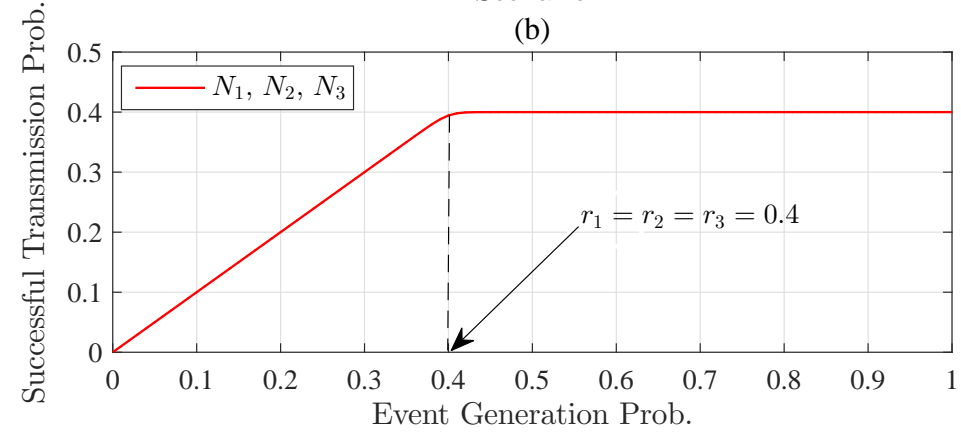

Figure 6.5: Figure (a) shows the network throughput for all the scenarios shown in Table 6.1. Figure (b) shows the successful transmission probability of $N_{1}, N_{2}$, and $N_{3}$. Parameters used: $q_{1}=q_{2}=q_{3}=0.2, \boldsymbol{p}_{1}=\boldsymbol{p}_{2}=\boldsymbol{p}_{3}=\left[\begin{array}{ll}0.2 & 0.8\end{array}\right], \tilde{K}=1, K=100, N=10^{5}, \delta_{1}=\delta_{2}=\delta_{3}=2$, $\tilde{\delta}=1$. 


\subsubsection{Fixed Transmit Power}

Using the first set of parameters contained in Figure 6.4, the results of the brute force approach in Figure 6.4 (a) show that $\Gamma$ is maximized at two scenarios. First, it is maximized at Scenario 3 where node 1 is a type $S_{1}$, node 2 is a cooperating node of node 3 . Second, it is maximized at Scenario 5 where node 2 is a type $S_{1}$, node 1 is a cooperating node of node 3. Thus, the optimal solution should be either Scenario 3 or Scenario 5 . In the centralized algorithm, from Figure $6.4(\mathrm{~b})$, we have that $q_{1}<r_{1}, q_{2}<r_{2}$, and $q_{3}>r_{3}$, which means that node 1 and node 2 are cooperating node candidates and node 3 is a type $S_{2}$ candidate. Because $d_{1}=d_{2}$, in the simulation, node 1 was randomly selected to be ordered ahead of node 2 in the set of cooperating node candidates. And because $\tau_{1}\left(v_{1}, \delta_{1}, \boldsymbol{p}_{1}\right) \approx 0.36>q_{1}+\tau_{3}\left(q_{3}, \delta_{3}, \boldsymbol{p}_{3}\right)=0.3$ events/time blocks, node 3 was assigned to be a type $S_{2}$ of node 1 , and node 2 was assigned to be a type $S_{1}$, which is the nodes' assignment of Scenario 5. If node 2 was randomly ordered ahead of node 1, then the optimal solution would have been Scenario 3 instead of Scenario 5. In the distributed algorithm, because node 2 is the cooperating node candidate of the largest ID number, the optimal solution was Scenario 3.

Using the second set of parameters contained in Figure 6.5, the brute force approach results in Figure 6.5 (a) show that $\Gamma$ is maximized at Scenario 1, where all the nodes are type $S_{1}$ nodes. In the centralized algorithm, this optimal solution was found without testing any of the nodes since all of them are cooperating node candidates as shown in Figure 6.5 (b). According to Proposition 1, if all the nodes are cooperating node candidates, then $\Gamma$ is maximized when all the nodes are type $S_{1}$ nodes. In the distributed algorithm, the optimal solution was found in the first time block because there was no $\tau(\cdot)$ s received at the cooperating node candidates and no ACK was broadcasted.

\subsubsection{Variable Transmit Power}

Using the third set of parameters shown in Figure 6.6, we simulate the scenario when the transmit power between the nodes is variable using the centralized algorithm. For comparison purposes, we also simulate the scenario when the transmit power is fixed. In the fixed 


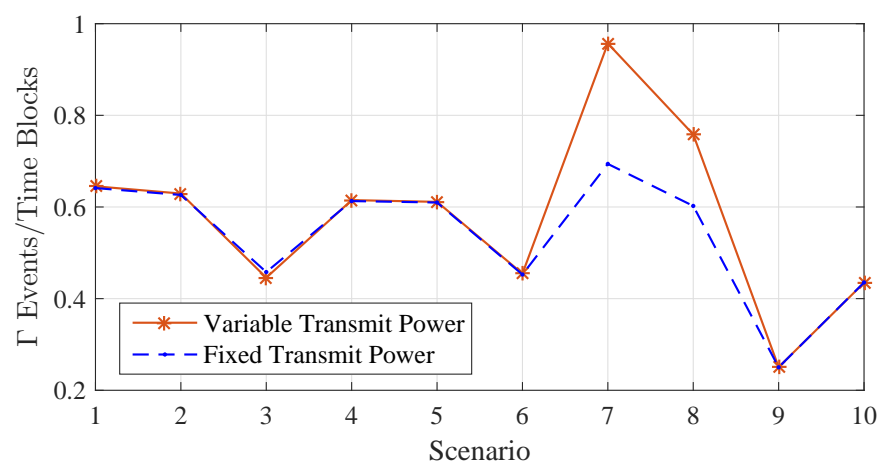

Figure 6.6: The network throughput for each scenario shown in Table 6.1 when the transmit power is variable and fixed. Parameters used: $q_{1}=0.2, q_{2}=0.8, q_{3}=0.2, \boldsymbol{p}_{1}=[0.0250 .025$ 0.0250 .0250 .10 .8 , $\boldsymbol{p}_{2}=\left[\begin{array}{llllll}0.2 & 0.3 & 0.3 & 0.2 & 0 & 0\end{array}\right], \boldsymbol{p}_{3}=\left[\begin{array}{llllll}0.1 & 0.1 & 0.2 & 0.3 & 0.3 & 0\end{array}\right], \tilde{K}=5, K=$ $100, N=10^{5}, \delta_{1}=\delta_{2}=\delta_{3}=6, \delta_{1,2}=2, \delta_{1,3}=4, \delta_{2,3}=1$.

transmit power scenario, in order for the nodes to communicate with each other, $\tilde{\delta}$ was set to 4, which is $\delta_{1,3}$, the transmit power between node 1 and node 3 . Using the same parameters, we also simulate the two transmit power scenarios using the brute force approach.

The results of the brute force approach in Figure 6.6 show that the network throughput is maximized at Scenario 7, where node 3 is a type $S_{1}$ and node 2 is a type $S_{2}$ of node 1 . Using the centralized algorithm, in the fixed transmit power scenario, this optimal solution was found in one iteration while in the variable transmit power scenario, it was found in two iterations. This is expected because in the variable transmit power scenario, each type $S_{2}$ candidate has to be tested as a type $S_{2}$ of each cooperating node candidate, and here the number of cooperating node candidates is 2. Also, the results show that the maximum throughput achieved when the transmit power is variable is higher than the maximum throughput achieved when the transmit power is fixed. This is because the transmit power between node 1 and node 2 in the fixed transmit power becomes higher, which results in less number of events that are successfully transmitted from node 2 to node 1 , and leads to a lower throughput. Finally, in the variable transmit power scenario, although the transmit power between node 1 and node 2 is higher than the transmit power between node 2 and node 3 , node 1 was selected to be a cooperating node of node 2 instead of node 3 . This implies that selecting a cooperating node for a node depends only on how many events that cooperating node can forward for that node. 


\subsubsection{Comparison with Cooperative Transmission}

We compare our approach with the cooperative transmission scenario when each pair of nodes in the network form a three-node relay channel with the base station, where one of the pair is a type $S_{2}$ node while the other is a cooperating node. We use the first and second sets of parameters shown in Figure 6.4 and Figure 6.5, respectively. Because the network has only three nodes, we consider that two of the nodes form a three-node relay channel with the base station while the third operates as a type $S_{1}$. In both cooperative transmission and our approach, the node that operates as a type $S_{1}$ achieves the same performance, which makes the comparison fair.

In cooperative transmission, when using the first set of parameters contained in Figure 6.4, we found that the network throughput is maximized when the nodes' assignment is set as in Scenario 3 or Scenario 5. In these two scenarios, because the type $S_{2}$ node always transmits cooperatively using the cooperating node that is always energized, the maximum throughput is the same as in our approach. However, in cooperative transmission, because the cooperating node gives priority for the type $S_{2}$ node's transmissions over its own, the cooperating node's throughput drops to 0.16 events/time blocks while the type $S_{2}$ node's throughput increases to 0.2 events/time blocks. In our case, the cooperating node's throughput stays the same that is 0.2 events/time blocks while the type $S_{2}$ node's throughput increases to 0.16 events/time blocks. This implies that in our approach, the overall throughput of the network is maximized such that no node's throughput is adversely affected.

As for the second set of parameters contained in Figure 6.5, when using cooperative transmission, we found that the network throughput is maximized when the nodes' assignment is set as in one of the scenarios from 2-7. From Figure 6.5 (a), the maximum throughput achieved in each of these scenarios is less than the maximum throughput achieved in our approach, which occurs when all the nodes are type $S_{1}$ nodes. The maximum throughput achieved in our approach can be achieved in cooperative transmission without affecting any node's throughput only if in the same time block, the cooperating node is allowed to transmit its own event after it forwards its type $S_{2}$ node's event to the base station. However, this will require increasing the length of time blocks, which will increase the overall time required 
to collect the same number of events compared to our approach.

\subsection{Conclusions}

In this chapter, we consider an uplink energy harvesting wireless sensor network. We address the problem of throughput maximization such that each node's throughput cannot drop below what it achieves by direct transmission to the base station. We propose centralized and distributed algorithms, and we consider fixed and variable transmit power scenarios. We conclude that when the transmit power between the nodes is variable, the maximum throughput can sometimes be higher than the maximum throughput achieved when the transmit power is fixed. However, when the transmit power is fixed, the computation complexity is lower. Compared with using cooperative transmission, our approach maximizes the network throughput such that no node's throughput is adversely affected. 


\section{Chapter 7}

\section{Summary and Future Work}

\subsection{Summary}

In this dissertation, we address the problem of transmission scheduling in cooperative energy harvesting sensor networks. We first consider the problem for a single cooperating node network. We propose a scheduling scheme, called a feedback scheme, that enables the source to decide whether to transmit its events to the destination by its own or by the help of the cooperating node such that the packet delivery ratio of the system is maximized. The feedback scheme can be generalized to any network of many sensor nodes when each group of three nodes in the network form a three-node relay channel. Compared with the-stateof-the-art scheme, the feedback scheme maximizes the packet delivery ratio of the system with no need for optimizing any of the system parameters. And thus, the feedback scheme becomes more practical in networks of unstable parameters and in networks of sensors with large battery capacity.

We then generalize the feedback scheme to include the case of multiple cooperating nodes, and to include one-way and two-way cooperative communications scenarios. The scheme can be extended to three or more hops where in each hop the transmitting node selects a cooperating node from the next hop using the same algorithm. However, this requires some changes in the system model. For example, the number of time slots per a time block would need to be adjusted. We also propose an adaptive method that reduces the overhead caused by transmitting the energy status of the cooperating nodes to the source 
in a separate feedback message. In addition, we propose an adaptive scheme that achieves fairness in the performance between the transmitting and cooperating nodes. The adaptive scheme considers only the case of absolute fairness, where the nodes have to achieve the same performance. This could be unreasonable in some scenarios when the penalty on the cooperating node performance has to be high in order to achieve the same performance of the source node. In addition, if the source node achieves higher performance than the cooperating node, the absolute fairness cannot be achieved as each node is fixed and categorized a priori as either a source or a cooperating node. Therefore, we next generalize the system model where each node in the network can be either a source or a cooperating node depending on the system parameters. We then propose a fairness scheduling (FS) scheme that ensures fairness in the performance of the nodes depending on a given penalty function, which fairly determines how much one of the nodes should cooperate with the other. We also propose a constrained scheduling (CS) scheme that constrains one of the transmitting nodes to achieve a certain performance, if possible.

Although the adaptive method reduces the overhead, it is still relatively high which could consume a significant amount of energy resulting in a lower performance of the system. Therefore, we also propose a statistical model that enables the source to estimate the energy status of the cooperating node. In the scenarios when the energy status of the cooperating node is deterministic, e.g., when the cooperating node is always energized, it will be more energy efficient to use the feedback because there will be no overhead. Otherwise, it will be more energy efficient to use the statistical model. We lastly address the problem of throughput maximization in an uplink energy harvesting wireless sensor network. We propose centralized and distributed algorithms that find the optimal role of each node, which is either source, relay, or user, such that the throughput is maximized. We consider fixed and variable transmit power scenarios and address complexity issues. We conclude that when the transmit power between the nodes is variable, the maximum throughput can sometimes be higher than the maximum throughput achieved when the transmit power is fixed. However, when the transmit power is fixed, the computation complexity is lower. Compared with using cooperative transmission, our approach maximizes the network throughput such that none of the nodes' throughputs is adversely affected. 


\section{2 $\quad$ Future Work}

In this section, we provide some interesting issues that can be addressed in the future. For example, in Chapter 4, there are two interesting issues that are described as follows:

1. The proposed schemes in the chapter were designed such that they provide the optimal operating mode for the system that is fixed over the $N$ time blocks. With the assumption that only one event can be reported per time block, in some scenarios, this may lead to energy overflow, which can be exploited to achieve better performance. To avoid energy overflow, the scheduling schemes need to be redesigned such that the operating mode of the system is selected instantaneously at the start of each time block, with the assumption that more than one event can be reported per time block. So that in each time block, each transmitting node can be either a source, a cooperating, or a relay node, and the cooperating node can transmit its own event, if any, after helping with the source's event transmission. In order to implement this new scenario, the joint distribution of the two transmitting nodes energy battery levels in the cooperation mode needs to be reformed under the following assumptions:

(a) The events of the two transmitting nodes are not mutually exclusive.

(b) If the two transmitting nodes both have an event generated in the same time block, each node transmits its event on its own if each has sufficient energy for direct transmission.

(c) If the two transmitting nodes both have an event generated in the same time block, each node transmits its event first, and it then cooperates with the other node if sufficient energy remains and if the other node has energy that is sufficient only for relay transmission.

2. We assume that the length of each time block is sufficient to report only one event. So, if the cooperating/relay node has an event generated, its event will be dropped to assist with the transmission of the other node if it has an event generated. And this may lead to energy overflow. As stated earlier, this could be solved by allowing for 
reporting more than one event per time block. However, this might not be the best solution in applications when the time delay in reporting events is untolerated. So another solution is using energy transfer technology. Instead of spending some amount of its collected energy on forwarding another node's transmissions, in this technology, the cooperating/relay node transfers that energy frequently to the other node as a RF signal. Thus, the cooperating node can transmit its events without delay regardless of the source's event status, assuming that the nodes transmit on different frequency bands or orthogonally. To implement this idea, we can assume that the source node $N_{i}, i \in\{1,2\}$ collects energy from the cooperating node $N_{j}, j \in\{1,2\}, j \neq i$ whenever it transmits to the destination. As in [70], we can assume that the energy transfer efficiency is $0 \leqslant \eta \leqslant 1$, i.e., whenever the cooperating node transmits to the destination using $\delta$ amount of energy, $(\eta \delta)$ amount of energy enters the source's battery. Based on these assumptions, we reform the distribution of the energy battery levels of the nodes and then we drive their packet delivery ratios. After that, we maximize the sum of the packet delivery ratios of the nodes over $\delta$ for the given $\eta$ such that $\delta_{j} \leqslant \delta \leqslant K$, assuming that $\delta_{j}$ is the minimum amount of transmit energy required to successfully report the event of the cooperating node to the destination.

In Chapter 6, there are also some interesting issues that can be addressed to make the proposed algorithm more general. These issues include:

1. We consider in Chapter 6 the case when $K \gg \delta_{i}$. The other case when $K \geqslant \delta_{i}$ can also be considered in order to include applications when battery capacity of sensor nodes cannot be large. When $K \geqslant \delta_{i}$, as shown in Section 6.3, the successful transmission probability has an additional region called transient. When a node operates in this range, it has energy overflow, and this energy can be exploited by operating the node as a type $S_{2}$ node so it transmits with lower energy and more events will be transmitted, or as a cooperating node to forward events to some other node/nodes. Thus, the nodes that operate in the transient region can be called cooperating-type $S_{2}$ candidates. To solve the problem, we first need to create a new set that includes all cooperating-type $S_{2}$ candidates, and then we need to modify the algorithm of the case when $K \gg \delta_{i}$ 
such that it tests these nodes as cooperating nodes first and then as type $S_{2}$ nodes.

2. The scenario that a node, in each time block, can make an instantaneous decision on its transmission path can be investigated as it may lead to a better throughput. 


\section{References}

[1] C. F. Garc'1a-Hern'andez et al., "Wireless Sensor Networks and Applications: a Survey," Int. J. Comput. Sci. Network Security, vol. 7, no. 3, pp. 264-273, Mar. 2007.

[2] J. Laneman, D. Tse, and G. Wornell, "Cooperative diversity in wireless networks: Efficient protocols and outage behavior," IEEE Trans. Inf. Theory, vol. 50, no. 12, pp. 3062-3080, Dec. 2004.

[3] M.O. Hasna, and M.S. Alouini, "End-to-end performance of transmission systems with relays over Rayleigh-fading channels," IEEE Transactions on Wireless Communications, vol.2, no.6, pp.1126-1131, Nov. 2003.

[4] D.S. Michalopoulos, and G.K. Karagiannidis, "Two-relay distributed switch and stay combining," IEEE Transactions on Communications, vol.56, no.11, pp.1790-1794, Nov. 2008.

[5] E. C. van der Meulen, "Three-terminal communication channels," Adv. Appl. Prob., vol. 3, pp. 120-154, 1971.

[6] T. Cover, and A. El Gamal, "Capacity theorems for the relay channel," IEEE Trans. Inf. Theory, vol. 25, no. 5, pp. 572-584, Sep. 1979.

[7] Y. Yao, X. Cai, and G. Giannakis, "On Energy Efficiency and Optimum Resource Allocation of Relay Transmissions in the Low-Power Regime," IEEE Transactions on Wireless Communication., vol. 4, no. 6, Nov. 2005.

[8] J. A. Paradiso, and T. Starner, "Energy scavenging for mobile and wireless electronics," IEEE Pervasive Computing, vol.4, no.1, pp.18-27, Jan.-Mar. 2005.

[9] X. Jiang, J. Polastre, and D. Culler, "Perpetual environmentally powered sensor networks," Fourth International Symposium on Information Processing in Sensor Networks, pp.463-468, 15 Apr. 2005.

[10] W. K. G. Seah, Z. A. Eu, and H. P. Tan, "Wireless sensor networks powered by ambient energy harvesting (WSN-HEAP) - Survey and challenges," 1st International Conference on Wireless Communication, Vehicular Technology, Information Theory and Aerospace and Electronic Systems Technology (Wireless VITAE 2009), Aalborg, pp. 1-5, 2009. 
[11] S. Chalasani, and J. M. Conrad, "A Survey of Energy Harvesting Sources for Embedded Systems," IEEE SoutheastCon, Huntsville, AL, pp. 442-447, Apr. 2008.

[12] B. Warneke, B. Atwood, and K. S. J. Pister, "Smart Dust mote forerunners," Proceedings of 14th Annual International Conference on Microelectromechanical Sytsems, pp.357-360, 2001.

[13] N.J. Guilar, T.J. Kleeburg, A. Chen, D.R. Yankelevich, and R. Amirtharajah, "Integrated Solar Energy Harvesting and Storage," IEEE Transactions on Very Large Scale Integration (VLSI) Systems, vol.17, no.5, pp.627-637, May 2009.

[14] C. Park, and P.H. Chou, "AmbiMax: Autonomous Energy Harvesting Platform for Multi-Supply Wireless Sensor Nodes," 3rd Annual IEEE Communications Society on Sensor and Ad Hoc Communications and Networks, 2006. SECON '06. pp.168-177, 28 Sep. 2006.

[15] B.W. Cook, S. Lanzisera; and K.S.J. Pister, "SoC Issues for RF Smart Dust," Proceedings of the IEEE, vol.94, no.6, pp.1177-1196, June 2006.

[16] P.D. Mitcheson, E.M. Yeatman, G.K. Rao, A.S. Holmes, and T.C. Green, "Energy Harvesting From Human and Machine Motion for Wireless Electronic Devices," Proceedings of the IEEE , vol.96, no.9, pp.1457-1486, Sep. 2008.

[17] T. Torfs, V. Leonov, C. Van Hoof, and B. Gyselinckx, "Body-Heat Powered Autonomous Pulse Oximeter," 5th IEEE Conference on Sensors, pp.427-430, 22-25 Oct. 2006.

[18] S. Sudevalayam, and P. Kulkarni, "Energy Harvesting Sensor Nodes: Survey and Implications," in IEEE Communications Surveys \& Tutorials, vol. 13, no. 3, pp. 443-461, Third Quarter 2011.

[19] H. Tan, P.W.Q. Lee, W.K.-G. Seah, and Z. A. Eu, "Impact of Power Control in Wireless Sensor Networks Powered by Ambient Energy Harvesting (WSN-HEAP) for Railroad Health Monitoring," International Conference on Advanced Information Networking and Applications Workshops, 2009. WAINA '09. pp.804-809, 26-29 May 2009.

[20] Z. A. Eu, W. K. G. Seah, and H. P. Tan, "A study of MAC schemes for wireless sensor networks powered by ambient energy harvesting," Accepted for publication for the 4th Wireless Internet Conference (WICON), Nov. 2008.

[21] J. Song, and Y. K. Tan, "Energy consumption analysis of ZigBee-based energy harvesting wireless sensor networks," IEEE International Conference on Communication Systems (ICCS), pp. 468-472, Singapore, 2012.

[22] X. Fafoutis, T. Sørensen, and J. Madsen, "Energy Harvesting - Wireless Sensor Networks for Indoors Applications Using IEEE 802.11", Procedia Computer Science, Vol. 32, pp. 991-996, ISSN 1877-0509, 2014. 
[23] R. Vaze, "Transmission capacity of wireless ad hoc networks with energy harvesting nodes," IEEE Global Conference on Signal and Information Processing (GlobalSIP), pp. 353-358, Austin, TX, 2013.

[24] D. Niyato, E. Hossain, and A. Fallahi, "Sleep and wakup strategies in solar-powered wireless sensor/mesh networks: Performance analysis and optimization," IEEE Transaction on Mobile Computation, vol. 6, pp. 221-236, Feb. 2007.

[25] C. Ok, H. Thadakamalla, U. Raghavan, S. Kumara, S. Kim, X. Zhang; and S. Bukkapatnam, "Optimal Transmission Power in Self-sustainable Sensor Networks for Pipeline Monitoring," IEEE International Conference on Automation Science and Engineering, 2007. CASE 2007. pp.591-596, 22-25 Sept. 2007.

[26] S. Mao, M. H. Cheung, and V.W.S. Wong, "An optimal energy allocation algorithm for energy harvesting wireless sensor networks," IEEE International Conference on Communications (ICC). pp.265-270, 10-15 June 2012.

[27] K. Tutuncuoglu, and A. Yener, "Optimum Transmission Policies for Battery Limited Energy Harvesting Nodes," IEEE Transactions on Wireless Communications, vol.11, no.3, pp.1180-1189, Mar. 2012.

[28] K. Tutuncuoglu, and A. Yener, "Short-Term Throughput Maximization for Battery Limited Energy Harvesting Nodes," IEEE International Conference on Communications (ICC), pp.1-5, 5-9 June 2011.

[29] O. Ozel, K. Tutuncuoglu, J. Yang; S. Ulukus, and A. Yener, "Adaptive transmission policies for energy harvesting wireless nodes in fading channels," 45th Annual Conference on Information Sciences and Systems (CISS), pp.1-6, 23-25 Mar. 2011.

[30] O. Ozel, K. Tutuncuoglu, J. Yang, S. Ulukus, and A. Yener, "Resource management for fading wireless channels with energy harvesting nodes," Proceedings IEEE INFOCOM, pp.456-460, 10-15 Apr.1 2011.

[31] A. Sendonaris, E. Erkip, and B. Aazhang, "User cooperation diversity. Part I. System description," IEEE Transactions on Communications, vol.51, no.11, pp.1927-1938, Nov. 2003.

[32] J.N. Laneman, W. Wornell, and D.N.C. Tse, "An efficient protocol for realizing cooperative diversity in wireless networks," IEEE International Symposium on Information Theory, pp.294, 2001.

[33] N. Devroye, P. Mitran, and V. Tarokh, "Achievable rates in cognitive radio channels," IEEE Transactions on Information Theory, vol.52, no.5, pp.1813-1827, May 2006.

[34] T.E. Hunter, and A. Nosratinia, "Diversity through coded cooperation," IEEE Transactions on Wireless Communications, vol.5, no.2, pp.283-289, Feb. 2006. 
[35] M. Janani, A. Hedayat, T.E. Hunter, and A. Nosratinia, "Coded cooperation in wireless communications: space-time transmission and iterative decoding," IEEE Transactions on Signal Processing, vol.52, no.2, pp.362-371, Feb. 2004.

[36] Bin Zhao and M. C. Valenti, "Practical relay networks: a generalization of hybrid-ARQ," in IEEE Journal on Selected Areas in Communications, vol. 23, no. 1, pp. 7-18, Jan. 2005.

[37] D.N. Nguyen, and M. Krunz, "Cooperative MIMO in wireless networks: recent developments and challenges," IEEE Network, vol.27, no.4, pp. 48-54, July-Aug. 2013.

[38] S. Shankar N, C. Chou, and M. Ghosh, "Cooperative communication MAC (CMAC) a new MAC protocol for next generation wireless LANs," International Conference on Wireless Networks, Communications and Mobile Computing, pp. 1-6, 2005.

[39] A. Azgin, Y. Altunbasak, and G. AlRegib, "Cooperative MAC and routing protocols for wireless ad hoc networks," GLOBECOM '05. IEEE Global Telecommunications Conference, 2005., St. Louis, MO, pp. 2854-2859, 2005.

[40] S. Moh, C. Yu, S. M. Park, H. N. Kim, and J. Park, "CD-MAC: Cooperative Diversity MAC for Robust Communication in Wireless Ad Hoc Networks," 2007 IEEE International Conference on Communications, pp. 3636-3641, Glasgow, 2007.

[41] P. Liu, Z. Tao, S. Narayanan, T. Korakis, and S. S. Panwar, "CoopMAC: A Cooperative $M A C$ for Wireless LANs," in IEEE Journal on Selected Areas in Communications, vol. 25, no. 2, pp. 340-354, Feb. 2007.

[42] S. Gupta, C.M. Vuran, and M.C. Gursoy, "Power efficiency of cooperative communication in wireless sensor networks," 3rd International Conference on Signal Processing and Communication Systems, 2009. ICSPCS 2009. pp.1-10, 28-30 Sept. 2009.

[43] Y. Chen, W. Yi, and Y. Yang, "A cross-layer strategy for energy-efficient cooperative communication in wireless sensor networks," International Conference on Wireless Sensor Network, 2010. IET-WSN. pp.203-208, 15-17 Nov. 2010.

[44] A. Mehrabi, and K. Kim, "Maximizing Data Collection Throughput on a Path in Energy Harvesting Sensor Networks Using a Mobile Sink," in IEEE Transactions on Mobile Computing, vol. 15, no. 3, pp. 690-704, Mar. 1, 2016.

[45] X. Ren, W. Liang, and W. Xu, "Data Collection Maximization in Renewable Sensor Networks via Time-Slot Scheduling," in IEEE Transactions on Computers, vol. 64, no. 7, pp. 1870-1883, July 1, 2015.

[46] X. Ren, and W. Liang, "Delay-tolerant data gathering in energy harvesting sensor networks with a mobile sink," IEEE Global Communications Conference (GLOBECOM), pp. 93-99, 2012. 
[47] R. S. Liu, K. W. Fan, Z. Zheng, and P. Sinha, "Perpetual and Fair Data Collection for Environmental Energy Harvesting Sensor Networks," in IEEE/ACM Transactions on Networking, vol. 19, no. 4, pp. 947-960, Aug. 2011.

[48] A. B. Sadlapur, and P. V. Pushpa, "Computing optimal data collection rate for energy harvesting sensor networks," 2013 International Conference on Advances in Computing, Communications and Informatics (ICACCI), pp. 1468-1472, Mysore, 2013.

[49] N. Dang, M. Roshanaei, E. Bozorgzadeh, and N. Venkatasubramanian, "Adapting data quality with multihop routing for energy harvesting wireless sensor networks," International Green Computing Conference Proceedings, pp. 1-6, Arlington, VA, 2013.

[50] X. Wang, V. S. Rao, R. V. Prasad, and I. Niemegeers, "Choose wisely: Topology control in Energy-Harvesting wireless sensor networks," 13th IEEE Annual Consumer Communications \& Networking Conference (CCNC), pp. 1054-1059, Las Vegas, NV, 2016.

[51] Y. Luo, J. Zhang, and K. B. Letaief, "Throughput maximization for twohop energy harvesting communication systems," IEEE International Conference on Communications (ICC), pp. 4180-4184, Budapest, 2013.

[52] B. Varan, and A. Yener, "Two-hop networks with energy harvesting: The (non-)impact of buffer size," in IEEE Global Conference on Signal and Information Processing (GlobalSIP), pp.399-402, 3-5 Dec. 2013.

[53] H. Li, N. Jaggi, and B. Sikdar, "An analytical approach towards cooperative relay scheduling under partial state information," IEEE Proceedings INFOCOM, pp.2666-2670, 25-30 Mar. 2012.

[54] K. H. Liu, "Performance Analysis of Relay Selection for Cooperative Relays Based on Wireless Power Transfer With Finite Energy Storage," in IEEE Transactions on Vehicular Technology, vol. 65, no. 7, pp. 5110-5121, July 2016.

[55] F. Wang, S. Guo, Y. Yang, and B. Xiao, "Relay Selection and Power Allocation for Cooperative Communication Networks With Energy Harvesting," in IEEE Systems Journal, vol.PP, no.99, pp.1-12, 2016.

[56] K. Liu, "Selection cooperation using RF energy harvesting relays with finite energy buffer," IEEE Wireless Communications and Networking Conference (WCNC), pp.2156-2161, 6-9 Apr. 2014.

[57] Y. Luo, J. Zhang, and K.B. Letaief, "Relay selection for energy harvesting cooperative communication systems," in IEEE Global Communications Conference (GLOBECOM), pp.2514-2519, 9-13 Dec. 2013.

[58] B. Medepally, and N.B. Mehta, "Voluntary Energy Harvesting Relays and Selection in Cooperative Wireless Networks," IEEE Transactions on Wireless Communications, vol.9, no.11, pp.3543-3553, Nov. 2010. 
[59] A.A. Nasir, X. Zhou, S. Durrani, and R.A. Kennedy, "Relaying Protocols for Wireless Energy Harvesting and Information Processing," IEEE Transactions on Wireless Communications, vol.12, no.7, pp.3622-3636, July 2013.

[60] I. Krikidis, S. Timotheou, and S. Sasaki, "RF Energy Transfer for Cooperative Networks: Data Relaying or Energy Harvesting?," IEEE Communications Letters, vol.16, no.11, pp.1772-1775, Nov. 2012.

[61] M. Tacca, P. Monti, and A. Fumagalli, "Cooperative and reliable ARQ protocols for energy harvesting wireless sensor nodes," IEEE Transaction on Wireless Communication, vol. 6, no. 7, pp. 2519-2529, July 2007.

[62] C. Huang, R. Zhang, and S. Cui, "Throughput Maximization for the Gaussian Relay Channel with Energy Harvesting Constraints," in IEEE Journal on Selected Areas in Communications, vol. 31, no. 8, pp. 1469-1479, Aug. 2013.

[63] N. Ishitani, K. Kobayashi, H. Okada, and M. Katayama, "Cooperative repeaters to improve data collection in low power generation for solarpowered wireless sensor networks," in 12th Annual IEEE Consumer Communications and Networking Conference (CCNC), pp.361-366, 9-12 Jan. 2015.

[64] S. Gong, L. Duan, and N. Gautam, "Optimal Scheduling and Beamforming in Relay Networks With Energy Harvesting Constraints," in IEEE Transactions on Wireless Communications, vol. 15, no. 2, pp. 1226-1238, Feb. 2016.

[65] A. Seyedi, and B. Sikdar, "Modeling and analysis of energy harvesting nodes in wireless sensor networks," 46th Annual Allerton Conference on Communication, Control, and Computing, pp.67-71, 23-26 Sept. 2008.

[66] E. Sazonov, H. Li, D. Curry, and P. Pillay, "Self-Powered Sensors for Monitoring of Highway Bridges," in IEEE Sensors Journal, vol. 9, no. 11, pp. 1422-1429, Nov. 2009.

[67] D. H. Ha, D. Kim, J. F. Choo, and N. S. Goo, "Energy harvesting and monitoring using bridge bearing with built-in piezoelectric material," The 7th International Conference on Networked Computing (INC), pp. 129-132, Gyeongsangbuk-do, 2011.

[68] W. Liu, Z. Wang, S. Qu, and R. Luo, "Vibration energy harvesting and management for wireless sensor networks in bridge structural monitoring," IEEE SENSORS, pp. 1-4, Busan, 2015.

[69] P. Yu, J Lee, T.Q.S. Quek, and Y.-W.P. Hong, "Energy harvesting personal cells - traffic offloading and network throughput," in IEEE International Conference on Communications (ICC), pp.2184-2189, 8-12 June 2015.

[70] B. Gurakan, O. Ozel, J. Yang, and S. Ulukus, "Energy cooperation in energy harvesting wireless communications," IEEE International Symposium on Information Theory Proceedings (ISIT), pp. 965-969, Cambridge, MA, 2012. 
[71] B. Gurakan, O. Kaya, and S. Ulukus, "Energy harvesting cooperative multiple access channel with data arrivals," IEEE International Conference on Communications (ICC), pp. 1-6, Kuala Lumpur, 2016. 